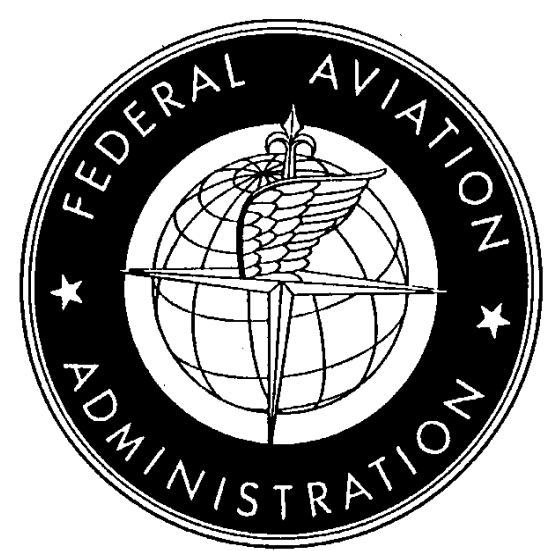

Federal Aviation Administration

Human Factors Team

Report on:

\title{
The Interfaces Between Flightcrews and Modern Flight Deck Systems
}

June 18, 1996 



\section{Foreword}

On April 26, 1994, an Airbus A300-600 operated by China Airlines crashed at Nagoya, Japan, killing 264 passengers and flightcrew members. Contributing to the accident were conflicting actions taken by the flightcrew and the airplane's autopilot. The crash provided a stark example of how a breakdown in the flightcrew/automation interface can affect flight safety. Although this particular accident involved an A300-600, other accidents, incidents, and safety indicators demonstrate that this problem is not confined to any one airplane type, airplane manufacturer, operator, or geographical region. This point was tragically demonstrated by the crash of a Boeing 757 operated by American Airlines near Cali, Columbia on December 20,1995, and a November 12, 1995 incident (very nearly a fatal accident) in which an American Airlines Douglas MD-80 descended below the minimum descent altitude on approach to Bradley International Airport, CT, clipped the tops of trees, and landed short of the runway.

As a result of the Nagoya accident, as well as other incidents and accidents that appear to highlight difficulties in flightcrews interacting with flight deck automation, the FAA's Transport Airplane Directorate, under the approval of the Director, Aircraft Certification Service, launched a study to evaluate the flightcrew/flight deck automation interfaces of current generation transport category airplanes. This report is the culmination of that study. 
This page intentionally left blank 


\section{Preface from the Co-Chairpersons}

This report is the result of a study of the interfaces between the flightcrew and the automated systems on highly automated airplanes. It primarily focuses on the interfaces that affect flight path management. The report was produced by a team of highly qualified individuals from the FAA and the European Joint Aviation Authorities, assisted by expert technical advisors from the Ohio State University, the University of Illinois, and the University of Texas. The co-chairs would like to commend their fellow team members and technical advisors for their special efforts, recognizing that everyone involved had to fit this extensive study into already difficult schedules. We also wish to thank the manufacturers, operators, pilots' associations, and researchers who met with us for supporting this important safety initiative.

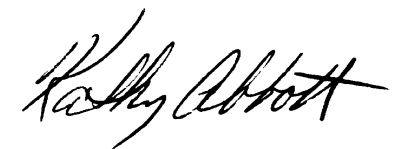

Dr. Kathy Abbott Co-chair, Human Factors Team NASA

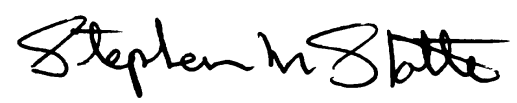

Stephen M. Slotte Co-chair, Human Factors Team FAA

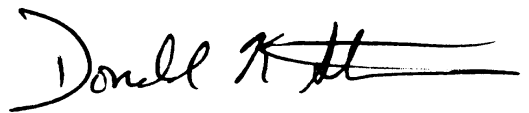

Donald K. Stimson Co-chair, Human Factors Team FAA 
This page intentionally left blank 
FAA Human Factors Team

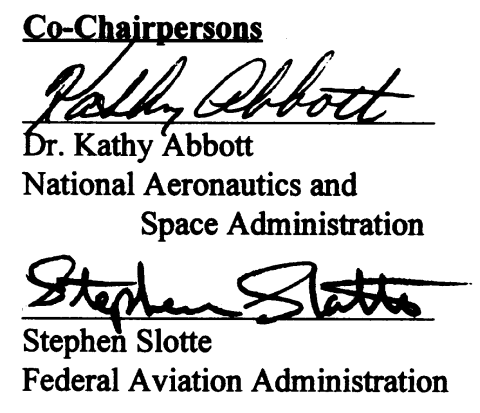

Federal Aviation Administration

Gene Boll in

Share tech Sharon Hecht

Merman mascle

Thomas Imrich

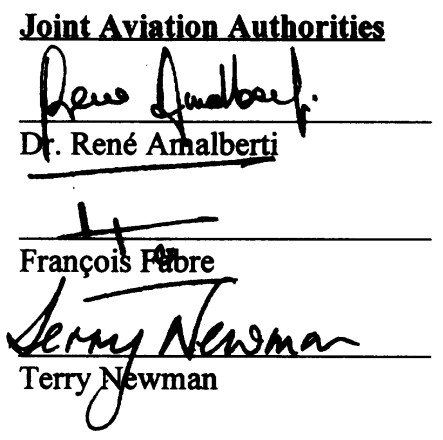

Expert Technical Advisors

Naduie Sorter

University of Illinois

Polar Hehmenct)

Dr. Robert Helmreich

University of Texas
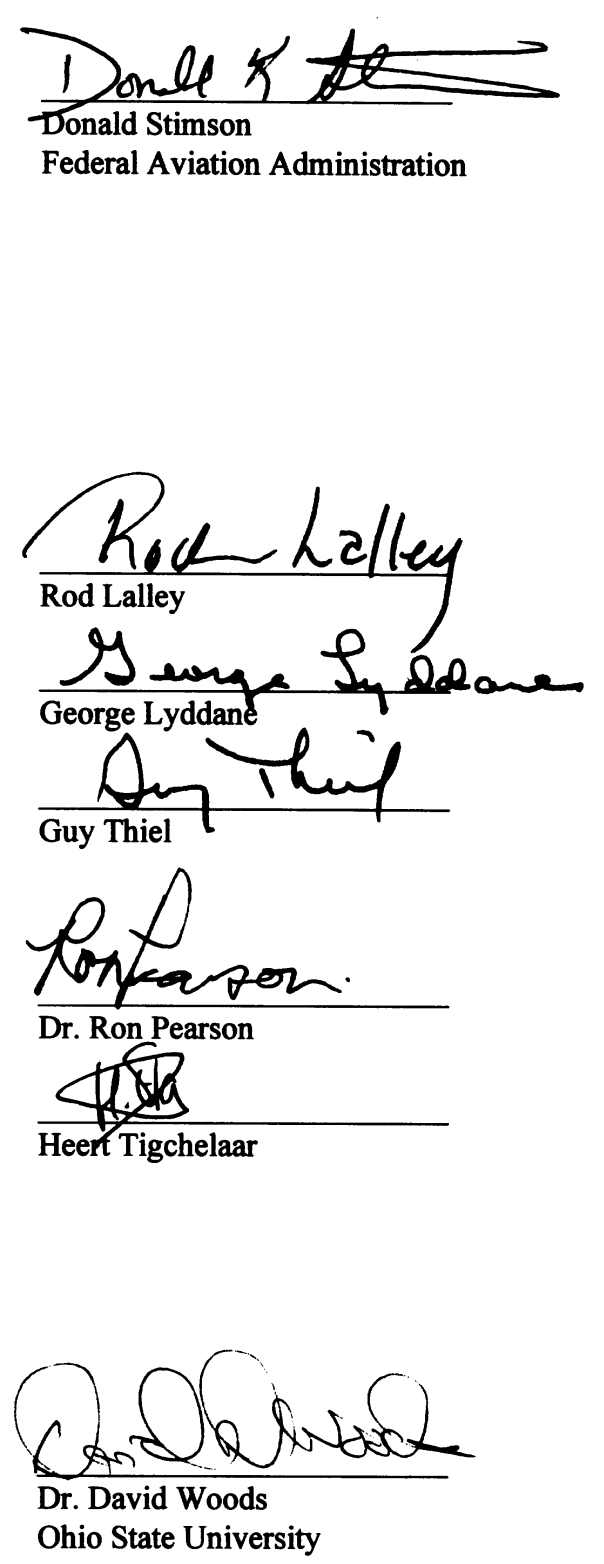

Page v 


\section{Acknowledgments}

The Human Factors Team thanks the many companies, organizations, and individuals who made valuable contributions to the successful completion of this study. Without their willing cooperation and desire to increase the safety of air transportation, this report would not have been possible. Specifically, the Team would like to express our gratitude to the following companies and organizations, and the individuals from these companies and organizations who participated in this study: Air France, Airbus Industrie, Airline Pilots Association, American Airlines, Allied Pilots Association, Air Transport Association of America, Boeing Commercial Airplane Group, British Airways, Crew System Ergonomics Information Analysis Center, Direction Générale de l'Aviation Civile (France), Douglas Aircraft Company, Federal Aviation Administration, European Joint Aviation Authorities, Fokker Aircraft B.V., Honeywell, Japan Civil Aviation Bureau, National Aeronautics and Space Administration, National Transportation Safety Board, Rijksluchtvaartdienst (Netherlands), Royal Aeronautical Society/Guild of Air Pilots and Air Navigators, United Air Lines, and the United Kingdom Civil Aviation Authority.

In addition, the Team members wish to acknowledge the contributions made towards this effort by the following individuals:

$\begin{array}{lll}\text { Terence Abbott } & \text { David Foyle } & \text { William Rogers } \\ \text { Doug Arbuckle } & \text { Richard Gifford } & \text { Vic Riley } \\ \text { Charles Billings } & \text { Howard "Berk" Greene } & \text { Marianne Rudisill } \\ \text { Monica Burgess } & \text { Forrest Keller } & \text { Paul Schutte } \\ \text { Katherine Burks } & \text { Victor Lebacqz } & \text { Tim Seeley } \\ \text { Sandi Carli } & \text { Alan Midkiff } & \text { David Simmon } \\ \text { Susan Conry } & \text { Kandy Mulrony } & \text { Michael Shafto } \\ \text { Bill Corwin } & \text { Ev Palmer } & \text { Rose Upton } \\ \text { Sheryl Chappell } & \text { Michael Palmer } & \text { Earl Wiener } \\ \text { Noel Duncan } & \text { Peter Polson } & \text { Lynn Williams } \\ \text { Sharon Flint } & & \end{array}$

We would also like to acknowledge the anonymous independent reviewers of initial drafts of this report for their valuable input. 


\section{Contents}

Executive Summary

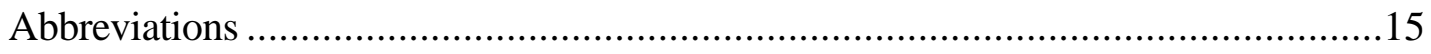

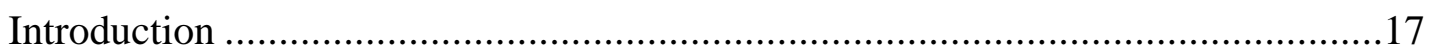

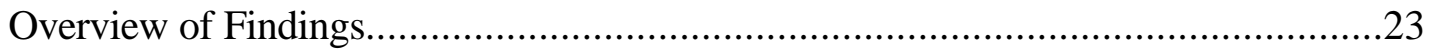

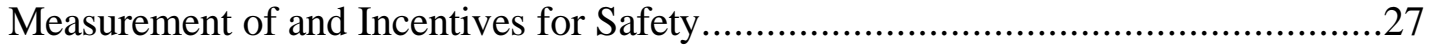

Flightcrew Management and Direction of Automation .............................................33

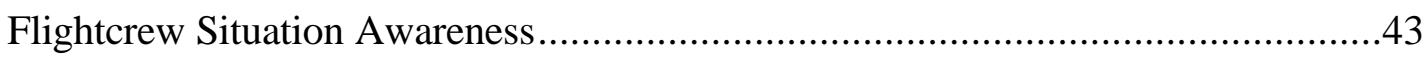

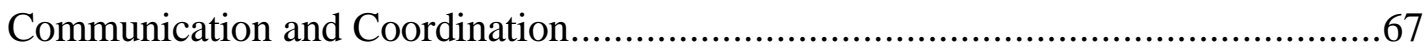

Processes for Design, Regulatory, and Training Activities.........................................81

Criteria, Regulatory Standards, Methods, and Tools for Design and Certification....95

Knowledge and Skills of Designers, Pilots, Operators, Regulators,

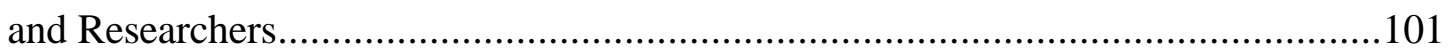

Cultural and Language Differences ................................................................117

Potential Barriers to Implementation of the Recommendations...............................123

Follow-On Effort and Implementation of the Recommendations ............................127

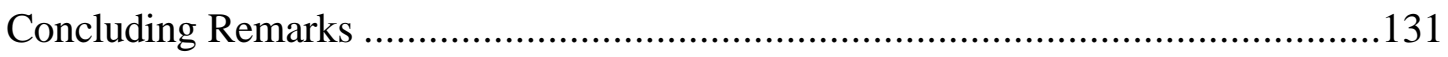

Appendices

Appendix A: Human Factors Team Charter Statement ......................................

Appendix B: Matrix of Issues and Recommendations .......................................

Appendix C: Supporting Data and References .................................................

Appendix D: Examples of Incidents and Accidents Involving the Flightcrew-

Automation Interface..............................................................

Appendix E: Existing FAR Part 25 Regulations and Advisory Circulars Related to Human Factors ...........................................................

Appendix F: Excerpts from the Aviation Safety Reporting System.....................F-1

Appendix G: Questions Use to Guide Discussions with Manufacturers and

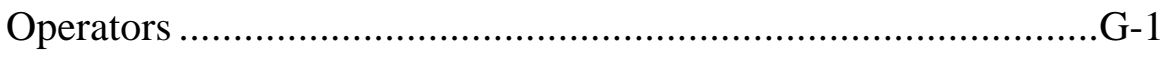




\section{Figures}

Figure 1

Interrelationships Between Issues and the Means to Address those Issues ........19

Figure 2

Representation of Autoflight Modes Displayed on Boeing Model 747-400

Primary Flight Display

Figure 3

Mode Selector Panels 51

Figure 4

Takeoff/Go-Around and Autothrottle Quick Disconnect Switch Locations .......54

Figure 5

Sample of Various Formatting Conventions for a Given Geographic Fix ...........55

Figure 6

Example Design Process Incorporating Human Factors Design Principles........91

Figure 7

Proposal for FAA Human Factors Team Follow-on Effort 


\section{Executive Summary}

Advances in technology have enabled increasingly sophisticated automation to be introduced into the flight decks of modern airplanes. Generally, this automation was added to accomplish worthy objectives such as reducing flightcrew workload, adding additional capability, or increasing fuel economy. To a large extent, these objectives have been achieved. Safety also stood to benefit from the increasing amounts of highly reliable automation. Indeed, the current generation of highly automated transport category airplanes has generally demonstrated an improved safety record relative to the previous generation of airplanes. Vulnerabilities do exist, though, and further safety improvements should be made. To provide a safety target to guide the aviation industry, the Secretary of Transportation and others have expressed the view that the aviation industry should strive for the goal of zero accidents.

On April 26, 1994, an Airbus A300-600 operated by China Airlines crashed at Nagoya, Japan, killing 264 passengers and flightcrew members. Contributing to the accident were conflicting actions taken by the flightcrew and the airplane's autopilot. The crash provided a stark example of how a breakdown in the flightcrew/automation interface can affect flight safety. Although this particular accident involved an A300-600, other accidents, incidents, and safety indicators demonstrate that this problem is not confined to any one airplane type, airplane manufacturer, operator, or geographical region. This point was tragically demonstrated by the crash of a Boeing 757 operated by American Airlines near Cali, Columbia on December 20, 1995, and a November 12, 1995 incident (very nearly a fatal accident) in which a American Airlines Douglas MD-80 descended below the minimum descent altitude on approach to Bradley International Airport, CT, clipped the tops of trees, and landed short of the runway.

As a result of the Nagoya accident as well as other incidents and accidents that appear to highlight difficulties in flightcrews interacting with the increasing flight deck automation, the Federal Aviation Administration's (FAA) Transport Airplane Directorate, under the

approval of the Director, Aircraft Certification Service, launched a study to evaluate the flightcrew/flight deck automation interfaces of current generation transport category airplanes. The following airplane types were included in the evaluation:

Boeing: Models 737/757/767/747-400/777

Airbus: Models A300-600/A310/A320/A330/A340

McDonnell Douglas: Models MD-80/MD-90/MD-11

Fokker: Model F28-0100/-0070

The FAA chartered a human factors (HF) team to address these human factors issues, with representatives from the FAA Aircraft Certification and Flight Standards Services, the National Aeronautics and Space Administration, and the Joint Aviation Authorities (JAA), assisted by technical advisors from the Ohio State University, the University of 
Illinois, and the University of Texas. The HF Team was asked to identify specific or generic problems in design, training, flightcrew qualifications, and operations, and to recommend appropriate means to address these problems. In addition, the HF Team was specifically directed to identify those concerns that should be the subject of new or revised Federal Aviation Regulations (FAR), Advisory Circulars (AC), or policies.

The HF Team relied on readily available information sources, including accident/incident reports, Aviation Safety Reporting System reports, research reports, and trade and scientific journals. In addition, meetings were held with operators, manufacturers, pilots' associations, researchers, and industry organizations to solicit their input. Additional inputs to the HF Team were received from various individuals and organizations interested in the HF Team's efforts.

When examining the evidence, the HF Team found that traditional methods of assessing safety are often insufficient to pinpoint vulnerabilities that may lead to an accident. Consequently, the HF Team examined accident precursors, such as incidents, errors, and difficulties encountered in operations and training. The HF Team also examined research studies that were intended to identify issues and improve understanding of difficulties with flightcrew/automation interaction.

In examining flightcrew error, the HF Team recognized that it was necessary to look beyond the label of flightcrew error to understand why the errors occurred. We looked for contributing factors from design, training and flightcrew qualification, operations, and regulatory processes. While the HF Team was chartered primarily to examine the flightcrew interface to the flight deck systems, we quickly recognized that considering only the interface would be insufficient to address all of the relevant safety concerns. Therefore, we considered issues more broadly, including issues concerning the functionality of the underlying systems.

From the evidence, the HF Team identified issues that show vulnerabilities in flightcrew management of automation and situation awareness. Issues associated with flightcrew management of automation include concerns about:

- Pilot understanding of the automation's capabilities, limitations, modes, and operating principles and techniques. The HF Team frequently heard about automation "surprises," where the automation behaved in ways the flightcrew did not expect. "Why did it do that?" "What is it doing now?" and "What will it do next?" were common questions expressed by flightcrews from operational experience.

- Differing pilot decisions about the appropriate automation level to use or whether to turn the automation on or off when they get into unusual or non-normal situations (e.g., attempted engagement of the autopilot during the moments preceding the A310 crash at Bucharest). This may also lead to potential mismatches with the manufacturers' assumptions about how the flightcrew will use the automation. 
Flightcrew situation awareness issues included vulnerabilities in, for example:

- Automation/mode awareness. This was an area where we heard a universal message of concern about each of the aircraft in our charter.

- Flight path awareness, including insufficient terrain awareness (sometimes involving loss of control or controlled flight into terrain) and energy awareness (especially low energy state).

These vulnerabilities appear to exist to varying degrees across the current fleet of transport category airplanes in our study, regardless of the manufacturer, the operator, or whether accidents have occurred in a particular airplane type. Although the Team found specific issues associated with particular design, operating, and training philosophies, we consider the generic issues and vulnerabilities to be a larger threat to safety, and the most important and most difficult to address. It is this larger pattern that serves as a barrier to needed improvements to the current level of safety, or could threaten the current safety record in the future aviation environment. It is this larger pattern that needs to be characterized, understood, and addressed.

In trying to understand this larger pattern, the Team considered it important to examine why these vulnerabilities exist. The Team concluded that the vulnerabilities are there because of a number of interrelated deficiencies in the current aviation system:

- Insufficient communication and coordination. Examples include lack of communication about in-service experience within and between organizations; incompatibilities between the air traffic system and airplane capabilities; poor interfaces between organizations; and lack of coordination of research needs and results between the research community, designers, regulators, and operators.

- $\quad$ Processes used for design, training, and regulatory functions inadequately address human performance issues. As a result, users can be surprised by subtle behavior or overwhelmed by the complexity embedded in current systems operated within the current operating environment. Process improvements are needed to provide the framework for consistent application of principles and methods for eliminating vulnerabilities in design, training, and operations.

- Insufficient criteria, methods, and tools for design, training, and evaluation. Existing methods, data, and tools are inadequate to evaluate and resolve many of the important human performance issues. It is relatively easy to get agreement that automation should be human-centered, or that potentially hazardous situations should be avoided; it is much more difficult to get agreement on how to achieve these objectives.

- Insufficient knowledge and skills. Designers, pilots, operators, regulators, and researchers do not always possess adequate knowledge and skills in certain areas related to human performance. It is of great concern to this team that investments in necessary levels of human expertise are being reduced in response to economic pressures when two-thirds to three-quarters of all accidents have flightcrew error cited as a major factor. 
- Insufficient understanding and consideration of cultural differences in design, training, operations, and evaluation. The aviation community has an inadequate understanding of the influence of culture and language on flightcrew/automation interaction. Cultural differences may reflect differences in the country of origin, philosophy of regulators, organizational philosophy, or other factors. There is a need to improve the aviation community's understanding and consideration of the implications of cultural influences on human performance.

\section{Based on our investigations and examination of the evidence, these concerns represent more than a series of individual problems with individual, independent solutions. These concerns are highly interrelated, and are evidence of aviation system problems, not just isolated human or machine errors. Therefore, we need system solutions, not just point solutions to individual problems. To treat one issue (or underlying cause) in isolation will ultimately fail to fundamentally increase the safety of airplane operations, and may even decrease safety.}

The HF Team developed recommendations to address the vulnerabilities and deficiencies from a system viewpoint. Our consideration of human performance issues, however, was focused primarily on the flightcrew. We did not attempt to address human performance issues associated with other personnel involved in the aviation system, such as flight attendants, ground personnel, air traffic services personnel, or maintenance personnel.

Because the system is already very safe, any changes should be made carefully to avoid detracting from existing safety practices. The Team believes we must improve and institutionalize:

- Investments in people (designers, users, evaluators, and researchers). For example, flightcrew training investments should be re-balanced to ensure appropriate coverage of automation issues.

- Processes. It is important to improve how design, training, operations, and certification are accomplished. For example, regulatory authorities should evaluate flight deck designs for human performance problems.

- Tools and methods. New tools and methods need to be developed and existing ones improved to accompany the process improvements.

- Regulatory standards. Current standards for type certification and operations have not kept pace with changes in technology and increased knowledge about human performance. For example, flightcrew workload is the major human performance consideration in existing Part 25 regulations; other factors should be evaluated as well, including the potential for designs to induce human error and reduce flightcrew situation awareness.

This report contains detailed discussions of each vulnerability and deficiency area, together with the HF Team's recommendations for addressing them, and suggested approaches for implementing the recommendations. The recommendations are listed below. For a more 
complete understanding of the intent behind the recommendation, the relevant section of the report must be read in detail.

\section{Measurement of and Incentives for Safety}

Recommendation Measures-1: The FAA should:

- Lead the aviation community to use accident precursors increasingly and consistently as an additional measure of aviation safety;

- Work with industry to establish systems/processes for collecting precursor data and for tracking the influence of system changes (e.g., design changes, training changes) on safety; and

- Work with industry to investigate other means of assessing or communicating safety (e.g., ways of measuring errors intercepted, incidents or accidents prevented).

Recommendation Measures-2: In accident/incident investigations where human error is considered a potential factor, the FAA and the National Transportation Safety Board should thoroughly investigate the factors that contributed to the error, including design, training, operational procedures, the airspace system, or other factors. The FAA should encourage other organizations (both domestic and foreign) conducting accident/incident investigations to do the same. This recommendation should apply to all accident/incident investigations involving human error, regardless of whether the error is associated with a pilot, mechanic, air traffic controller, dispatcher, or other participant in the aviation system.

Recommendation Measures-3: The FAA should explore means to create additional incentives to improve safety through appropriate design, training, or operational improvements.

\section{Management of Automation}

Recommendation AutomationMgt-1: The FAA should ensure that a uniform set of information regarding the manufacturers' and operators' automation philosophies is explicitly conveyed to flightcrews.

Recommendation AutomationMgt-2: The FAA should require operators' manuals and initial/recurrent qualification programs to provide clear and concise guidance on:

- Examples of circumstances in which the autopilot should be engaged, disengaged, or used in a mode with greater or lesser authority;

- The conditions under which the autopilot or autothrottle will or will not engage, will disengage, or will revert to another mode; and 
- Appropriate combinations of automatic and manual flight path control (e.g., autothrottle engaged with the autopilot off).

Recommendation AutomationMgt-3: The FAA should initiate a review of the autopilots on all transport category airplanes to identify the potential for producing hazardous energy states, excessive pitch or bank angles, subtle departures from the intended flight path, slow-overs, hard-overs, or other undesirable maneuvers. Results of this review should be the basis for initiating appropriate actions, such as design improvements, flight manual revisions, additional operating limitations, or changes in training programs or operational procedures.

Recommendation AutomationMgt-4: The FAA should assure that analyses are conducted to better understand why flightcrews deviate from procedures, especially when the procedural deviation contributes to causing or preventing an accident or incident.

Recommendation AutomationMgt-5: The FAA should request industry to take the lead in developing design guidelines for the next generation of flight management systems.

\section{Flightcrew Situation Awareness}

Recommendation SA-1: The FAA should require operators to increase flightcrews' understanding of and sensitivity to maintaining situation awareness, particularly:

- Mode and airplane energy awareness issues associated with autoflight systems (i.e., autopilot, autothrottle, flight management system, and fly-by-wire flight control systems);

- Position awareness with respect to the intended flight path and proximity to terrain, obstacles, or traffic; and

- Potential causes, flightcrew detection, and recovery from hazardous pitch or bank angle upsets while under autopilot control (e.g., wake vortex, subtle autopilot failures, engine failure in cruise, atmospheric turbulence).

Recommendation SA-2: The FAA should require operators' initial and recurrent training programs as well as appropriate operating manuals to:

- Explicitly address autoflight mode and airplane energy awareness hazards;

- Provide information on the characteristics and principles of the autoflight system's design that have operational safety consequences; and

- Provide training to proficiency of the flight management system capabilities to be used in operations.

Recommendation SA-3: The FAA should encourage the aviation industry to develop and implement new concepts to provide better terrain awareness. 
Recommendation SA-4: The FAA and the aviation industry should develop and implement a plan to transition to standardized instrument approaches using lateral navigation (LNAV) and vertical navigation (VNAV) path guidance for three-dimensional approaches. The use of approaches that lack vertical path guidance should be minimized and eventually eliminated.

Recommendation SA-5: The FAA should encourage the exploration, development, and testing of new ideas and approaches for providing effective feedback to the flightcrew to support error detection and improved situation awareness.

Recommendation SA-6: The FAA should encourage standardization, as appropriate, of automation interface features, such as:

- The location, shape, and direction of movement for takeoff/go-around and autothrottle quick disconnect switches;

- Autoflight system mode selectors and selector panel layout,

- Autoflight system modes, display symbology, and nomenclature; and

- Flight management system interfaces, data entry conventions, and nomenclature.

Recommendation SA-7: The FAA and the aviation industry should update or develop new standards and evaluation criteria for information presented to the flightcrew by flight deck displays and aural advisories (e.g., primary flight displays, navigation/communication displays, synoptics showing system states).

Recommendation SA-8: The FAA should ensure that flightcrews are educated about hazardous states of awareness and the need for countermeasures to maintain vigilance. The FAA should encourage operators to:

- Develop operational procedures and strategies to foster attention management skills with the objective of avoiding hazardous states of awareness; and

- Develop techniques to apply during training to identify and minimize hazardous states of awareness.

Recommendation SA-9: The FAA should sponsor research, or assure that research is accomplished, to develop improved methods for:

- Evaluating designs for susceptibility to hazardous states of awareness (e.g., underload, complacency, absorption); and

- Training to minimize hazardous states of awareness. 


\section{Communication and Coordination}

Recommendation Comm/Coord-1: The FAA should identify existing air traffic procedures that are incompatible with highly automated airplanes. These incompatible procedures should be discontinued or modified as soon as feasible.

Recommendation Comm/ Coord-2: The FAA should task an existing advisory group or, if necessary, establish a new forum to ensure coordination between the design of air traffic procedures and the design and operation of highly automated airplanes.

Recommendation Comm/ Coord-3: The FAA should lead an industry-wide effort to share safety information obtained from in-service data and from difficulties encountered in training. This effort should be capable of assisting in the identification and resolution of problems attributed to flightcrew error.

Recommendation Comm/ Coord-4: The FAA should require operators to have an appropriate process, with demonstrated effectiveness, for informing flightcrews about relevant accidents, incidents, in-service problems, and problems encountered in training that could affect flight safety.

Recommendation Comm/ Coord-5: The FAA should encourage the redesign and modernization of the information provided to the flightcrew in notices to airmen (NOTAMs), charts, approach plates, instrument procedures, meteorological data, etc. The information should be prioritized and highlighted in terms of urgency and importance, and presented in a clear, well-organized, easy-to-understand format suitable for use with current and future airplanes.

Recommendation Comm/ Coord-6: The FAA should improve and increase interaction between the Flight Standards and Aircraft Certification Services.

Recommendation Comm/ Coord-7: The FAA and industry should improve the coordination and distribution of tasks undertaken by federal advisory committees and industry technical committees to reduce overlap and avoid duplication of effort.

Recommendation Comm/ Coord-8: The FAA should improve communication about research programs, research results, and advances in technology to appropriate FAA personnel.

Recommendation Comm/ Coord-9: The FAA should hold research funding sponsors and researchers accountable for supporting the transfer of research results.

Recommendation Comm/ Coord-10: The FAA should assure strategic leadership and support establishment of a coordinated research portfolio in aviation human factors on the national and international levels. 


\section{Processes for Design, Regulatory, and Training Activities}

Recommendation Processes-1: The FAA should task an aviation industry working group to produce a set of guiding principles for designers to use as a recommended practice in designing and integrating human-centered flight deck automation.

Recommendation Processes-2: The FAA should establish regulatory and associated advisory material to require the use of a flight deck certification review process that addresses human performance considerations.

Recommendation Processes-3: The FAA and the aviation industry should investigate the use of innovative training tools and methods to expand pertinent safety related knowledge of flightcrews on a continuing basis. The FAA and the aviation industry should explore incentives to encourage continued training and education beyond the minimum required by the current regulations.

\section{Criteria, Regulatory Standards, Methods and Tools for Design and Certification}

Recommendation Criteria-1: The FAA should require evaluation of flight deck designs for susceptibility to design-induced flightcrew errors and the consequences of those errors as part of the type certification process.

Recommendation Criteria-2: The FAA should prepare and distribute interim guidance material that updates current autopilot certification policy.

Recommendation Criteria-3: The FAA should task an appropriate Aviation Rulemaking Advisory Committee Harmonization Working Group (HWG) with updating the autopilot regulatory standards (14 CFR 25.1329). This HWG should include specialists

knowledgeable in human factors methods and skills from both industry and the regulatory authorities.

Recommendation Criteria-4: The FAA should revise/update the following specific FARs and associated advisory material:

- $\$ 25.1322$ Warning, caution, and advisory lights: Revise to reflect the current and anticipated design practice for modern transport category airplanes.

- $\quad \S 25.1335$ Flight Director: Revise to reflect the current and anticipated design practice for modern transport category airplanes.

- $\$ 121.703$ Mechanical reliability reports: Revise the requirements to also include reporting of significant flight deck automation failures and/or anomalies that adversely affect safe flight path management. Reinforce the Aviation Rulemaking Advisory Committee (ARAC) activity in this area. 


\section{Knowledge and Skills of Designers, Pilots, Operators, Regulators and Researchers}

Recommendation Knowledge-1: The FAA should encourage flight deck design organizations to:

(1) Make human factors engineering a core discipline of the flight deck system design activity; and

(2) Ensure that the design team has sufficient human factors and operational knowledge and expertise by:

- Distributing guiding principles for flightcrew-centered design (as described in Recommendation Processes-1) to all design team members;

- Including human factors expertise as part of the design team;

- Assuring that each member of the team has at least a basic knowledge of human factors in order to understand and communicate human performance issues and human-centered design considerations at some appropriate level; and

- Assuring that flight deck design team members have relevant operational knowledge.

Recommendation Knowledge-2: The FAA should reassess the requirements that determine the content, length, and type of initial and recurrent flightcrew training. Ensure that the content appropriately includes:

- Management and use of automation, including mental models of the automation and moving between levels of automation;

- Flightcrew situation awareness, including mode and automation awareness;

- Basic airmanship;

- Crew Resource Management;

- Decision making, including unanticipated event training;

- Examples of specific difficulties encountered either in service or in training; and

- Workload management (task management).

The FAA should work with industry to develop guiding principles and associated advisory material for training, operational procedures, and flightcrew qualification for the areas listed above.

Recommendation Knowledge-3: The FAA should strongly encourage or provide incentives to make advanced maneuvers training an integral part of the training curriculum, especially in recurrent training.

Recommendation Knowledge-4: The FAA should reassess recency requirements for flightcrews involved in long haul operations. Consider providing incentives and alternative 
methods for flightcrews to practice takeoffs and landings, and perhaps arrival and departure procedures that are infrequently used.

Recommendation Knowledge-5: The FAA should reassess the airman certification criteria to ensure that pilots are released with a satisfactory level of skills for managing and using automation. Since current training is often oriented toward preparing pilots for checkrides, the airman certification criteria should be reassessed to ensure appropriate coverage of the topics listed in Recommendation Knowledge-2.

Recommendation Knowledge-6: Operators should ensure that flight safety and training managers are appropriately educated about human factors considerations, particularly with regard to automation.

Recommendation Knowledge-7: The FAA should improve the education of Air Traffic Service personnel about the capabilities and limitations of highly automated airplanes.

Recommendation Knowledge-8: The FAA should provide appropriate regulatory personnel with a guide or roadmap to current Federal Aviation Regulations, advisory material, policy memoranda, and other guidance material dealing with human performance related to the flightcrew-system interface. The FAA should ensure that this material is used in aircraft certification projects, airline qualification program assessments, and airman qualification.

Recommendation Knowledge-9: The FAA should develop a systematic training program for appropriate Aircraft Certification and Flight Standards Services personnel to provide initial and recurrent training in the area of human factors as it relates to certifying new products and evaluating flightcrew performance. The training should include instruction on:

- Insight into the relationship among the flightcrew, the flight deck design, and the operation environment;

- Flightcrew information processing;

- Workload, human error, and situation awareness;

- Other flightcrew performance issues, including fatigue, CRM, and attention management;

- Design and evaluation of flight deck displays;

- Aircraft control laws and feedback systems;

- Human-automation interaction;

- Human-centered design principles and guidelines; and

- Ergonomics -- fitting the design to the user. 
Recommendation Knowledge-10: The FAA should appropriately staff the standards organizations and aircraft certification offices with human factors expertise and integrate personnel with such expertise into certification teams, participating and applying their expertise in the same manner as other certification team members (e.g., airframe, flight test, systems and equipment, propulsion).

Recommendation Knowledge-11: The FAA should increase Aircraft Certification and Flight Standards Services personnel's knowledge about each other's roles and responsibilities. In particular, increase certification pilots' and engineers' knowledge of line operations considerations, and Aircraft Evaluation Group personnel's knowledge about airworthiness certification considerations.

Recommendation Knowledge-12: The FAA should improve the knowledge of personnel in Aircraft Certification and Flight Standards Services about processes for identifying and communicating requirements for research (either specific studies required or identification of areas of concern).

Recommendation Knowledge-13: The FAA should encourage researchers to learn more about industry and FAA's research needs and about operational considerations in aviation.

\section{Cultural and Language Differences}

Recommendation Culture-1: The FAA should ensure that research is conducted to characterize cultural effects and provide better methods to adapt design, training, publications, and operational procedures to different cultures. The results of the research should also be used to identify significant vulnerabilities, if any, in existing flight deck designs, training, or operations, and how those vulnerabilities should be addressed.

Recommendation Culture-2: The FAA should encourage simplified flight deck messages, training, manuals, and procedures with clearer meaning to non-native English speakers. The FAA should encourage the use of internationally understood visual symbols and pictures where appropriate, rather than verbal descriptions or directions.

Recommendation Culture-3: The FAA should provide leadership to update ICAO phraseology standards and to encourage their use.

Recommendation Culture-4: The FAA should promote timely and clear communications between flightcrews and Air Traffic Services through:

- Accelerated efforts for transmission of information via datalink, as appropriate (e.g., Automated Terminal Information System (ATIS), weather, pre-departure clearances);

- Assuring clear and intelligible transmission of ATIS and clearance information, where datalink is unavailable or unsuitable; and 
- Standard procedures and taxi routes.

Implementing the HF Team's recommendations will not be easy; many of the recommendations call for institutional or organizational changes that may generate resistance. However, the Team considers these changes necessary in order to achieve the reduction in the accident rate sought by the public and the aviation community. The HF Team recommends that the FAA form a follow-on team and task it with coordinating the implementation of these recommendations. This team should provide guidance to affected FAA organizations, and should work with industry, industry groups, the JAA, and other airworthiness authorities to assist in carrying out the recommendations.

The HF Team recognizes the economic pressures that inhibit making changes that may increase safety when there is not a strong tie to an accident. However, we believe that if action is not taken soon, the vulnerabilities identified have the potential to lead to more accidents and serious incidents. 
This page intentionally left blank 


\section{Abbreviations}

Following are abbreviations used in this report:

AC Advisory circular

ACO Aircraft certification office

$\mathrm{AD} \quad$ Airworthiness directive

AEG Aircraft Evaluation Group

ALPA Airline Pilots Association

APA Allied Pilots Association

AQP Advanced Qualification Program

ARAC Aviation Rulemaking Advisory Committee

ASAP Aviation Safety/Accident Prevention

ASRS Aviation Safety Reporting System

ATA Air Transport Association of America

ATC Air Traffic Control

ATIS Automatic Terminal Information Service

ATS Air Traffic Services

AWO All weather operations

CFIT Controlled flight into terrain

CMO Certificate Management Office

CNS Communication, Navigation, and Surveillance

CRM Crew resource management

DGAC Direction Générale de l'Aviation Civile (France)

FAA Federal Aviation Administration

FAR Federal Aviation Regulations

FCOM Flightcrew operating manual

FCU Flight control unit

FMS Flight management system

FOEB Flight Operations Evaluation Board

FSB Flight Standardization Board

FSDO Flight Standards District Office

GPS Global Positioning System

GPWS Ground Proximity Warning System

HF Human factors 
HFStG Human factors steering group (JAA)

HWG Harmonization working group

ICAO International Civil Aviation Organization

IFR Instrument Flight Rules

IOE Initial Operational Experience

ILS Instrument Landing System

JAA Joint Aviation Authorities

JAR Joint Aviation Requirements

LNAV Lateral navigation

LOFT Line Oriented Flight Training

LOS Line Operational Simulations

NASA National Aeronautics and Space Administration

NOAA National Oceanic and Atmospheric Administration

NOTAM Notice to Airmen

NTSB National Transportation Safety Board

PDC Pre-departure clearance

PFD Primary flight display

RLD Rijksluchtvaartdienst (The Netherlands Civil Aviation Agency)

RNP Required Navigation Performance

SAE Society of Automotive Engineers

STC Supplemental type certificate

TAD Transport Airplane Directorate

TC Type certificate

TCAS Traffic Alert and Collision Avoidance System

VNAV Vertical navigation

VOR Very High Frequency Omnidirectional Radio Range 


\section{Introduction}

\section{Background}

By traditional safety measures (e.g., accidents per million departures), air travel is considered to be very safe. For the last 20 years, the accident rate for the worldwide commercial jet fleet has remained fairly constant at about 2 to 3 accidents per million departures. ${ }^{1}$ Over that same time period, however, the number of worldwide departures per year has almost doubled, going from about 8 million to over 15 million. ${ }^{2}$ With this traffic growth expected to continue, more accidents will occur each year unless the accident rate is reduced. Since public confidence in the safety of air travel appears to be determined by the aggregate number of accidents occurring over a given time period, continued public confidence demands that the accident rate be reduced. Also, in order to provide a safety target to guide the aviation industry, the Secretary of Transportation, Frederico Peña, has expressed the view that the aviation industry should strive for the goal of zero accidents.

Accident statistics cite the flightcrew as a primary contributor in over 60 percent of accidents involving transport category airplanes. ${ }^{3}$ The introduction of modern flight deck designs, which have automated many piloting tasks, has reduced or eliminated some types of flightcrew errors, but other types of errors have been introduced. Several recent accidents and incidents have emphasized continuing difficulties in flightcrew interaction with flight deck automation. Other indicators of potential safety problems, such as flightcrew reports, training and operational difficulties, research studies, and surveys also point to vulnerabilities in this area.

In response to increasing concerns over the flightcrew/airplane interfaces, the FAA's Transport Airplane Directorate (TAD) formed the Human Factors Team (HF Team) to evaluate the vulnerability of the current fleet to breakdowns in flightcrew/airplane interaction. A study was initiated to consider all aspects influencing the flightcrew's ability to safely use the displays and automated systems dealing with flight path management. ${ }^{4}$

\footnotetext{
${ }^{1}$ Statistical Summary of Commercial Jet Aircraft Accidents. Boeing Commercial Airplane Group, April, 1996.

2 ibid.

$3_{\text {ibid. }}$

${ }^{4}$ Flight path management is defined as the integration of guidance, navigation, control and associated interfaces/control devices used by the pilot to direct or control the flight path of the aircraft.
} 
The following airplane types were included in the evaluation:

Boeing: Models 737/757/767/747-400/777

Airbus: Models A300-600/A310/A320/A330/A340

McDonnell Douglas: Models MD-80/MD-90/MD-11

Fokker: Model F28-0100/-0070

Although this evaluation specifically focused on these airplane types and considered primarily air carrier operations, the HF Team's findings are generic in nature and can be applied to other transport category airplanes as well as business, executive, and commuter airplanes. The types included in the evaluation were chosen because they represent the majority of the highly automated airplanes currently being operated by the major air carriers, and because they are under the purview of this study's sponsoring organization.

\section{Human Factors Team Charter}

\section{Statement of Objectives:}

The Team will evaluate current generation transport category airplane flight deck designs in regard to the human interfaces with airplane systems and the effect of these interfaces on airplane safety. The study will concentrate on the design, training/flightcrew qualification, and operation of those systems dealing with flight path management. The Team will consider all factors that can influence the pilot's ability to safely operate the airplane during all phases of flight, including, but not limited to, mode/situation awareness, pilot expectations regarding the automatic systems and the subsequent pilot response when those expectations are not met, and crew resource management in modern flight decks.

The Team shall:

a) Identify specific and generic safety related design problems, if any, related to pilot/airplane interfaces, in the airplane types under study. The Team will recommend appropriate means to address these problems.

b) Identify specific and generic training/flightcrew qualification and operational problems, if any, related to pilot/airplane interfaces in the airplane types under study. The Team will recommend appropriate means to address these problems.

c) Identify those concerns that should be the subject of new or revised Federal Aviation Regulations (FAR), advisory circulars (AC) and/or policies.

\section{Report Scope}

The HF Team was chartered to consider all aspects of the flightcrew/airplane interface affecting flight path management. The HF Team was asked to identify specific or generic 
problems in design, training, flightcrew qualifications, and operations, and to recommend appropriate means to address these problems. In addition, the HF Team was specifically directed to identify those concerns that should be the subject of new or revised Federal Aviation Regulations (FAR), Advisory Circulars (AC), or policies. Figure 1 illustrates the inter-relationships between the issues studied by the HF Team and the means considered for addressing them.

\section{ISSUES UNDER STUDY}

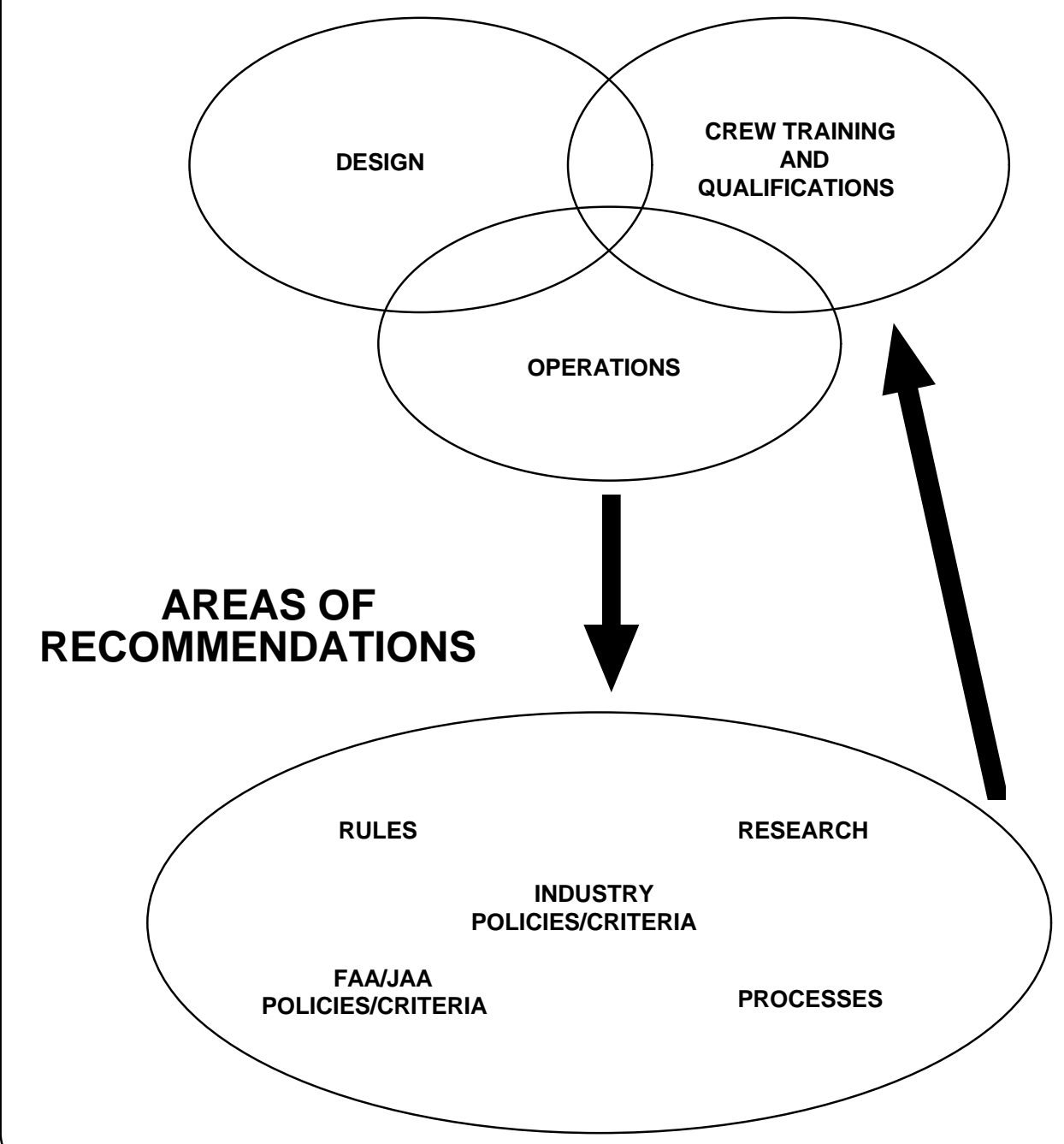

Figure 1

Interrelationships Between Issues and the Means to Address those Issues 


\section{HF Team Composition}

Represented on the HF Team ${ }^{5}$ were:

- Two FAA National Resource Specialists

- Flight Management

- Air Carrier Operations

- NASA Aviation Human Factors Specialist

- Two FAA Flight Test Pilots

- FAA Flight Standards Pilot

- Two FAA Aircraft Certification Service aerospace engineers

- FAA Human Factors Specialist

- JAA Human Factors Specialist (and an alternate)

- Two JAA Flight Test Pilots (and an alternate)

- Independent Consultants to the Team:

- Human Factors Researcher from The Ohio State University

- Human Factors Researcher from the University of Illinois

- Crew Resource Management Researcher from the University of Texas

The HF Team actively sought and received input from recognized experts in the field and other interested parties, including industry and labor groups as well as government and academic sources. Widespread publicity of the HF Team's activity generated additional inputs. In addition, three expert technical advisors from the academic community were retained to provide direct assistance to the HF Team.

\section{Information Sources}

The HF Team relied on readily available information sources, including accident/incident reports, Aviation Safety Reporting System (ASRS) reports, research reports, and trade and scientific journals. In addition, meetings were held with operators, manufacturers, pilots' associations, researchers, and industry organizations to solicit their input. (Examples of questions used to guide the discussions in these meetings are provided in Appendix G.) Additional inputs to the HF Team were received from various individuals and organizations interested in the Team's efforts. With the limited time available, the HF Team did not conduct or sponsor additional research or studies. A list of references containing the major information sources and supporting data may be found in Appendix C.

\footnotetext{
${ }^{5}$ The final team composition listed above varied slightly from the listing contained in the team's charter. The Transport Airplane Directorate hired a human factors specialist after the study was underway, and this specialist was added to the HF Team. One of the independent consultants, whose affiliation was listed as The Ohio State University in the charter, took a position with the University of Illinois before the team finished its work. In addition, the two JAA representatives identified as alternates participated as full team members on an as available basis, and therefore, appear on the report's signature page.
} 


\section{Roadmap to Reading the Report}

The layout of this report is described below. To further assist the time-constrained reader, we offer the following roadmap to the report. A quick synopsis of the report's contents can be obtained from the section entitled "Overview of Findings." For further information about a specific issue area, including details of the team's findings and concerns, turn to the section on that issue area. Finally, a complete listing of the team's recommendations can be found in Appendix B.

Following this introduction section, we present an overview of the HF Team's findings. The "Overview of Findings" consists of a high-level overview of the safety issues arising from our study of breakdowns in flightcrew/automation interaction, why these safety issues exist, and the types of changes we consider necessary to address them. The overview section serves both as a foundation for the detailed discussion of specific issues in later sections, and as a summary and integration of the issue areas and means of addressing them.

Following the "Overview of Findings," we devote a separate section to each major issue area identified during the study. In each of these sections, we explain why the HF Team believes the issue represents a safety concern, and we provide specific examples to illustrate particular problem areas. At the end of each section are the HF Team's recommendations associated with the issue area discussed in that section. The recommendations are stated in a way that is intended to provide a desired objective rather than the specific means for accomplishing that objective. Additional discussion follows each recommendation to provide further detail and to suggest a means for implementing the recommendation, although we recognize that there may be other ways to achieve the desired result.

We recognize that there may not be universal support for all of our recommendations. Therefore, following the sections on specific issue areas, we devote a section to discussing the potential barriers to implementing the recommendations. Also in this section, we present the myths about human factors that tend to pervade the aviation community and impede progress in this important field.

Following the "Potential Barriers" section, we make suggestions regarding the follow-on effort that will be needed to implement the recommendations. We close the report with some concluding remarks that summarize the HF Team's findings and encourage the aviation industry to continue to commit itself to addressing human factors issues.

Appendices include: the team charter statement, a listing of the HF Team's recommendations, a summary of supporting data and references, examples of incidents and accidents involving the flightcrew/automation interface, a list of current Part 25 regulations and advisory material addressing human factors issues, excerpts of narratives from the ASRS, and the questions used to guide the discussions during our meetings with airplane operators and manufacturers. 
Page 22 


\section{Overview of Findings}

The aviation industry has an enviable and well-earned safety record, but this safety record can and should be improved even further. Given that flightcrew error is cited as a primary factor in such a large percentage of accidents involving transport category airplanes, addressing flightcrew error becomes a primary target for improving safety. Recent accidents and incidents highlight difficulties in the interaction between flightcrews and advanced flight deck automation. Recognition of these breakdowns in flightcrew/automation coordination was the major motivation for chartering the HF Team to determine whether the difficulties were associated with specific airplane types or whether there were generic problems associated with the current fleet of transport category airplanes.

When examining the available evidence, the Team found that traditional methods of assessing safety are often insufficient to pinpoint vulnerabilities that may lead to an accident. Consequently, the HF Team examined accident precursors, such as incidents, errors, and difficulties encountered in operations and training. The HF Team also examined research studies that were intended to identify issues and improve understanding of difficulties with flightcrew/automation interaction.

In examining flightcrew error, the HF Team recognized that it was necessary to look beyond the label of flightcrew error to understand why the errors occurred. We looked at the evidence for contributing factors from design, training and flightcrew qualification, operations, and regulatory processes. While the HF Team was primarily chartered to examine the flightcrew interface to the flight deck systems, we quickly recognized that considering only the interface would be insufficient to address all the relevant safety concerns. Therefore, we considered issues more broadly, including issues concerning the functionality of the underlying systems.

From the evidence, the HF Team identified issues that show vulnerabilities in flightcrew management of automation and situation awareness. Issues associated with flightcrew management of automation include concerns about:

- Pilot understanding of the automation's capabilities, limitations, modes, and operating principles and techniques. The HF Team frequently heard about automation "surprises," where the automation behaved in ways the flightcrew did not expect. "Why did it do that?" "What is it doing now?" and "What will it do next?" were common questions expressed by flightcrews from operational experience.

- Differing pilot decisions about the appropriate automation level to use or whether to turn the automation on or off when they get into unusual or non-normal situations (e.g., attempted engagement of the autopilot during the moments preceding the A310 crash at Bucharest). This may also lead to potential mismatches with the manufacturers' assumptions about how the flightcrew will use the automation. 
Flightcrew situation awareness issues included vulnerabilities in, for example:

- Automation/mode awareness. This was an area where we heard a universal message of concern about each of the aircraft in our charter.

- Flight path awareness, including insufficient terrain awareness (sometimes involving loss of control or controlled flight into terrain) and energy awareness (especially low energy state).

These vulnerabilities appear to exist to varying degrees across the current fleet of transport category airplanes in our study, regardless of the manufacturer, the operator, or whether accidents have occurred in a particular airplane type. Although the Team found specific issues associated with particular design, operating, and training philosophies, we consider the generic issues and vulnerabilities to be a larger threat to safety, and the most important and most difficult to address. It is this larger pattern that serves as a barrier to needed improvements to the current level of safety, or could threaten the current safety record in the future aviation environment. It is this larger pattern that needs to be characterized, understood, and addressed.

In trying to understand this larger pattern, the Team considered it important to examine why these vulnerabilities exist. The Team concluded that the vulnerabilities are there because of a number of interrelated deficiencies in the current aviation system:

- Insufficient communication and coordination. Examples include: lack of communication about in-service experience within and between organizations; incompatibilities between the air traffic system and airplane capabilities; poor interfaces between organizations; and lack of coordination of research needs and results between the research community, designers, regulators, and operators.

- $\quad$ Processes used for design, training, and regulatory functions inadequately address human performance issues. As a result, users can be surprised by subtle behavior or overwhelmed by the complexity embedded in current systems operated within the current operating environment. Process improvements are needed to provide the framework for consistent application of principles and methods for eliminating vulnerabilities in design, training, and operations.

- Insufficient criteria, methods, and tools for design, training, and evaluation. Existing methods, data, and tools are inadequate to evaluate and resolve many of the important human performance issues. It is relatively easy to get agreement that automation should be human-centered, or that potentially hazardous situations should be avoided; it is much more difficult to get agreement on how to accomplish these objectives.

- Insufficient knowledge and skills. Designers, pilots, operators, regulators, and researchers do not always possess adequate knowledge and skills in certain areas related to human performance. It is of great concern to this team that investments in necessary levels of human expertise are being reduced in response to economic pressures when two-thirds to three-quarters of all accidents have flightcrew error cited as a major factor. 
- Insufficient understanding and consideration of cultural differences in design, training, operations, and evaluation. The aviation community has an inadequate understanding of the influence of culture and language on flightcrew/automation interaction. Cultural differences may reflect differences in the country of origin, the philosophy of regulators, organizational aspects, or other factors. There is a need to improve the aviation community's understanding and consideration of the implications of cultural influences on human performance.

\section{Based on our investigations and examination of the evidence, these concerns represent more than a series of individual problems with individual, independent solutions. These concerns are highly interrelated, and are evidence of aviation system problems, not just isolated human or machine errors. Therefore, we need system solutions, not just point solutions to individual problems. To treat one issue (or underlying cause) in isolation will ultimately fail to fundamentally increase the safety of airplane operations, and may even decrease safety.}

The HF Team developed recommendations to address the vulnerabilities and deficiencies from a system viewpoint. Our consideration of human performance issues, however, was focused primarily on the flightcrew (although we did consider the operator's, manufacturer's, and researcher's perspective where appropriate). We did not attempt to address human performance issues associated with other personnel involved in the aviation system, such as flight attendants, ground personnel, air traffic services personnel, or maintenance personnel.

Because the system is already very safe, any changes should be made carefully to avoid detracting from existing safety practices. The Team believes we must improve and institutionalize:

- Investments in people (designers, users, evaluators, and researchers). For example, flightcrew training investments should be re-balanced to ensure appropriate coverage of automation issues.

- Processes. It is important to improve how design, training, operations, and certification are accomplished. For example, regulatory authorities should evaluate flight deck designs for human performance problems.

- Tools and methods. New tools and methods need to be developed and existing ones improved to accompany the process improvements.

- Regulatory standards. Current standards for type certification and operations have not kept pace with changes in technology and increased knowledge about human performance. For example, flightcrew workload is the major human performance consideration in existing Part 25 regulations; other factors should be evaluated as well, including the potential for designs to induce human error and reduce flightcrew situation awareness.

This report contains the Team's recommendations for improvements in each of these areas. Implementing the Team's recommendations will not be easy; many of the 
recommendations call for institutional or organizational changes that will generate resistance. However, the Team considers these changes necessary in order to achieve the reduction in the accident rate sought by the public and the aviation community.

While implementing these recommendations, the Team believes it is important to adhere to the following principles:

- Minimize human error. It is impossible to prevent all human error without removing the human flexibility and adaptability that contributes significantly to safety. Moreover, it is the negative consequences of error that we wish to eliminate, not necessarily the errors themselves. However, it is still desirable to minimize errors that are design or system induced.

- Increase error tolerance. The systems should be designed to aid the flightcrew to detect errors when they occur. Also, the systems should be designed such that errors that do occur have bounds on the undesirable consequences that result.

- Avoid excess complexity as perceived by the user. The systems should be designed to support the flightcrew, and should not be perceived as unnecessarily complex.

- Increase system observability, especially by improving system feedback.

- Evaluate new technology or operational changes introduced into the aviation system, especially the flight deck, for their effect on human performance.

- Invest in human expertise. This investment should include flightcrews, designers, operators, regulators, and researchers. We want to reinforce and strengthen the human contribution to safety in a proactive, rather than reactive, way.

The Team recognizes the economic pressures that inhibit making changes that may increase safety when there is not a strong tie to an accident. However, we believe that if action is not taken soon, the vulnerabilities identified have the potential to lead to more accidents and serious incidents. 


\section{Measurement of and Incentives for Safety}

Current measures of safety are typically based on accident rate (accidents per million departures) or number of accidents as the primary measure of safety. This information is useful and important, especially as a means of communicating safety information. Nonetheless, there are limitations to using only these measures, such as the inability to relate accident rates to specific areas of safety vulnerability. Furthermore, there are other means and motivations for assessing safety, related to accident prevention, for which the current measures are insufficient. The aviation community needs indicators of vulnerabilities that can serve as predictors of potential accidents, so that accident prevention does not depend on accidents occurring. Further, the aviation community must be able to evaluate the safety contributions of changes in design, training, operations, or regulatory practices.

The HF Team was tasked to identify potential problems related to flightcrew interfaces with advanced flight deck systems. Yet we heard numerous times opinions to the effect that "there hasn't been an accident in that aircraft caused by that particular design feature (or that training program, or that operational procedure). Therefore, it must be safe." Such a perspective ignores evidence of vulnerabilities, such as incident data, common errors encountered in operations, or difficulties in training that occur on a frequent basis, but that may not yet have resulted in or been identified as a contributing factor in an accident. Yet these other data may represent precursors to accidents. Most accidents have many precursors that might have led one to predict the accident. The challenge is to identify these precursors, minimize their individual risk, implement strategies that protect against broad classes of risk, and assure that specific chains of events containing these precursors cannot link up in unexpected ways that lead to an accident.

When analyzing accidents where pilot error is being investigated as a factor, it is too easy to label the cause as "pilot error." To prevent future accidents, it is critical to examine why the erroneous action or misassessment occurred. There are usually multiple factors that contribute to flightcrew errors, including deficiencies in design, training, manuals, procedures, or other factors (or a combination of factors). In many of the serious incidents and accidents involving flightcrew error, the triggering event initially appears to be minor. But through a series of misassessments and miscommunications between the flightcrew and the automation, the situation deteriorates into an accident or serious incident. With the benefit of hindsight, the chain of events often appears surprising. The information needed by the flightcrew to prevent the incident or accident appears to be obvious or logical. However, it clearly was not obvious or logical to the flightcrew who made the error. ${ }^{6}$

\footnotetext{
${ }^{6}$ For further discussion of this point, refer to Behind Human Error: Cognitive Systems, Computers and Hindsight by David Woods, Leila J. Johannesen, Richard I. Cook, and Nadine B. Sarter. CSERIAC SOAR 94-01, December, 1994.
} 
This type of incident or accident scenario seems to be a potential side effect of very complex systems. Fortunately, accidents are rare, because the deterioration is usually blocked by the expertise of the humans involved or characteristics of the system design. But sometimes circumstances do come together in a way that is not prevented, and an accident results. It is important to investigate the underlying factors and combination of circumstances that lead to a serious incident or accident involving human error in order to prevent it from happening again. This is true for all human errors (e.g., maintenance, air traffic personnel), not just flightcrew errors. There has been a growing trend towards performing this type of analysis by organizations such as the NTSB. The HF Team strongly endorses this trend.

Trying to solve these situations by only changing a particular design feature or providing additional training in a specific area overlooks the interrelated nature of these issues. While such changes may contribute to improving safety, it is important not to assume that a single solution to an individual aspect of an accident is sufficient. It is important to look at the entire set of factors (e.g., training and improved design may both be required; training cannot be viewed as a sole and permanent means to fix vulnerabilities in a design).

It can be very difficult to assess the contribution of specific changes or combinations of changes in design, training, operational procedures, or regulations to the traditional safety measures. For example, it is difficult to measure directly the effect on the accident rate of increasing training time or changing the content of training courses. A measure such as accident rate, while important, is not sensitive enough to give indications of the effects of incremental improvements. Therefore, we need additional measures to serve as safety indicators, especially for flightcrew performance and its contribution to overall system performance and safety.

Defining measures to provide more sensitive indications of system safety will not be easy. Nonetheless, it is imperative that some measures be determined. Currently, economic considerations are often favored when safety effects are hard to quantify, because there is a natural tendency to assume that something that can be easily quantified is intrinsically more important than something that cannot easily be quantified.

In general, the cost of any changes intended to improve safety usually gets more attention and emphasis than the benefit (e.g., accidents prevented), primarily because cost is easy to measure and quantify, and the effect of a change may be somewhat uncertain or hard to measure. One consequence of this difficulty in quantifying the benefit of improvements is that economic pressures reduce incentives for making these improvements, unless the safety improvement is obvious, immediate, quantifiable, or in reaction to an accident. Lack of perceived benefits or other incentives can delay or prevent safety improvements that otherwise might be implemented, and whose beneficial effects might be more apparent if different safety measures were used.

As an example of a situation where economic considerations are sometimes perceived to outweigh safety is the process for deciding whether to incorporate design or product 
improvements that are periodically offered by airplane manufacturers. Some of these improvements are believed by many in the operational community to be significant safety enhancements that would be a wise and justifiable investment. However, without clear economic benefits, improvements are unlikely to be implemented unless there is an accident or serious incident that spurs political pressure or issuance of an airworthiness directive (AD). In addition, the improvements that contribute to safety are sometimes offered together with other features that an operator does not want, making the purchase of the improvement more costly than the operator believes justifiable.

When ADs are issued, the manufacturer often pays for the improvements. Where service bulletins are issued but are not accompanied by an $\mathrm{AD}$, the operators often pay for the improvement. This method of assigning costs can lead to concerns that requiring or mandating improvements developed by manufacturers would discourage them from voluntarily developing improvements that contribute to both safety and economy. Conversely, not issuing an AD may lead to issuance of a service bulletin for which the operator will not or cannot pay. Incentives for some form of cost sharing could be a potentially useful approach to facilitating the incorporation of these types of improvements.

\section{Recommendations}

The Team recognizes the economic pressures that inhibit making changes that may increase safety when there is not a strong tie to an accident. Improved measures of safety may contribute to facilitating the incorporation of safety related improvements, even when there is a significant economic cost.

\section{Recommendation Measures-1}

The FAA should:

- Lead the aviation community to use accident precursors increasingly and consistently as an additional measure of aviation safety;

- Work with industry to establish systems/processes for collecting precursor data and for tracking the influence of system changes (e.g., design changes, training changes) on safety; and

- Work with industry to investigate other means of assessing or communicating safety (e.g., ways of measuring errors intercepted, incidents or accidents prevented, etc.). 


\section{Discussion of Recommendation Measures-1:}

The FAA Associate Administrator for Safety should lead this activity and solicit participation from airlines, airplane manufacturers, and other relevant organizations (e.g., the Flight Safety Foundation, the International Air Transport Association, and ICAO). Information from other industries should also be considered (e.g., nuclear power, railway, maritime, medicine, computer manufacturing, and any other industry using high levels of automation). This activity should be coordinated with the implementation of Recommendation Comm/Coord-3 (in-service data collection).

Many organizations in industry have safety departments that collect and disseminate such information. We recognize and wish to reinforce such activities. We recommend that successful examples of these activities be encouraged in organizations that do not have them already. We also encourage the sharing of information systematically among organizations in industry and government to a larger extent than is being done now.

We recognize that this recommendation will be very difficult to implement, and that the methods for analyzing the resulting data will greatly affect its usefulness. Potential barriers include concerns by industry about inappropriate release and use of information, compromise of competitive advantages, adverse publicity for those operators providing the most effective feedback, and cost. Another inhibitor to the sharing of safety data is resolving the issue of legal immunity for airlines and pilots. Evidence of the magnitude of these barriers is that the initial exchange of safety-related operational data among major U.S. airlines, scheduled to begin January 22, 1996, did not take place, ${ }^{7}$ mainly because of such legal concerns.

It also will not be easy to develop appropriate new measures for expressing the level of safety. Education may be required to expand the view of safety beyond simply the number or rate of accidents.

\section{Recommendation Measures-2}

In accident/incident investigations where human error is considered a potential factor, the FAA and the National Transportation Safety Board should thoroughly investigate the factors that contributed to the error, including design, training, operational procedures, the airspace system, or other factors. The FAA should encourage other organizations (both domestic and foreign) conducting accident/incident investigations to do the same. This recommendation should apply to all accident investigations involving human error, regardless of whether the error is associated with a pilot, mechanic, air traffic controller, dispatcher, or other participant in the aviation system.

\footnotetext{
7“U.S. Airlines Delay Exchange of Safety Data." Aviation Week and Space Technology. January 29, 1996, p 51.
} 


\section{Discussion of Recommendation Measures-2:}

As mentioned previously, it is inappropriate to attribute the cause of an incident or accident to human error and not investigate factors that may have contributed to the error being made. It is important to understand why the erroneous action or misassessment occurred. The FAA and the NTSB currently investigate these contributing factors as part of accident and incident investigations. The HF Team endorses the analysis of contributing factors, and recommends that all organizations conducting accident/incident investigations place even stronger emphasis on this analysis as an integral part of the investigative process.

\section{Recommendation Measures-3}

The FAA should explore means to create additional incentives to improve safety through appropriate design, training, or operational improvements.

\section{Discussion of Recommendation Measures-3:}

The FAA should lead this activity to develop additional incentives, with industry and other government agencies providing inputs and suggestions. It is very difficult to weigh economics against potential safety improvements that do not have a clear and direct safety benefit (unless a change is mandated), and great creativity will be needed to develop ideas for new incentives. As examples of past improvements, flight directors and autopilots were originally introduced to modern aircraft largely through providing the capability for low visibility landings, even though they ultimately had very significant safety benefits across the whole operational envelope. A valuable target for the future might be similar incentives to encourage operators to adopt relevant product improvements.

Such incentives could be financial, operational, or otherwise. Ideas should be solicited from specialists in organizational dynamics, regulatory policy making, and safety culture. Products of this activity might include new guidelines for defining a higher level of safety and agreements as to what incentives could be made available, how they would be funded, how they relate to the desired aircraft modification or capability, and what benefits might be realized.

The HF Team did not underestimate the difficulty of implementing this recommendation. It will be hard to develop effective new ideas. However, the potential benefits could be significant. 


\section{Flightcrew Management and Direction of Automation}

"Why did the FMS drop the fix/restriction? I don't really know."

"I failed to realize that the altitude restrictions are not in effect during a speed mode descent."

"Both of us were engrossed in trying to figure out why this computerized marvel was doing what it was, rather than turning everything off and manually flying (which we finally did) until we could sort things out."

“The captain then said, 'What's going on,' at which point the aircraft was observed 300 feet high; it had entered a subtle climb seemingly on its own accord...This is another case of learning to type 80 words a minute instead of flying the aircraft. The more automation there is in the aircraft, it just means the flightcrew should work that much harder to remain an active and integral part of the loop."

- Quotes from the ASRS database

The HF Team's assessment of flightcrew management of automation issues includes concerns in two major areas:

(1) Pilot understanding of the automation, its capabilities, behavior, modes of operation, and procedures for use; and

(2) Differing pilot decisions about the appropriate automation level to use (if any) in normal and non-normal circumstances. 


\section{Pilot Understanding of the Automation}

Automation surprises, where the automation behaves in ways the flightcrew does not expect or understand, are a too-frequent occurrence on highly automated airplanes. We heard this message in each of our meetings with operators' and pilots' organizations. It was also expressed in many of the research reports examined by the HF Team. Flightcrews are often faced with trying to answer the commonly asked questions about automation behavior, "Why did it do that?" "What is it doing now?" and "What will it do next?" We found that some of the automation surprises reflect an incomplete understanding of either the automation's capabilities and limitations, its display annunciations, or its intended use. Other surprises may reflect differences in the circumstances of use from those envisioned by the system designers.

From our investigations, the HF Team found that many flightcrews have difficulty understanding the autoflight system implementation of concepts such as speed-on-pitch (i.e., speed controlled by varying the airplane pitch attitude) and speed-on-thrust (i.e., speed controlled by varying the engine thrust level), even though these same basic concepts are also used in manual flight. If these concepts and their implementation are not well understood, flightcrews can easily become confused by autoflight system annunciations and behavior.

Complex automation interfaces, large differences in automation philosophy and implementation among different airplane types (including different airplane types from the same manufacturer as well as from different manufacturers), and inadequate training also contribute to deficiencies in flightcrew understanding of automation. An example of one of the HF Team's specific concerns in this area is the use of the flight management system's (FMS) vertical flight path modes. There is a general consensus that these modes are the most difficult for flightcrews to fully understand. Yet some operators provide very little training, if any, on the appropriate use of these modes. In these cases, flightcrews are expected to learn how to use the vertical modes during line operations.

The HF Team is very concerned about both the quality and the quantity of automation training flightcrews receive. (See the sections on "Processes for Design, Training, and Regulatory Activities" and "Knowledge and Skills of Designers, Pilots, Operators, Regulators, and Researchers" for additional discussion of training issues from both a process viewpoint and a knowledge and skills viewpoint.) In terms of overall training philosophy, there were differing views presented to the HF Team regarding training for automation. One view holds that flightcrews should be relieved of the burden of fully understanding system operation or the system's underlying design philosophy. This view ultimately leads to a training philosophy in which flightcrews are trained to respond primarily in a rote manner (i.e., very rigid operating procedures). The contrasting view is that flightcrews should be trained in the underlying principles of the system's design, leaving some of the details to individual good operating practice or technique. 
While the HF Team supports the use of standardized operating procedures as one effective strategy for managing error, we also consider it important for flightcrews to understand the principles and assumptions embodied in the automation design that affect safe operational use, especially where these principles and assumptions may differ from those of the flightcrew. In the absence of this understanding, flightcrews are likely to substitute their own model of how the automation works, based on their observations and assumptions of automation behavior. In some instances, the flightcrew's model will be incomplete or incorrect, leading to confusion and increasing the potential for error. In critical circumstances, such confusion can lead to a hazardous situation or at least make it difficult for the flightcrew to respond in an appropriate manner.

See the section entitled "Flightcrew Situation Awareness" for further discussion of the vulnerabilities in flightcrew situation awareness due to an incomplete understanding of the automation.

\section{Differing Pilot Decisions about Automation Use}

Recent incidents and accidents demonstrate that flightcrews differ in their use of automation when responding to an abnormal situation, and more importantly, may react in ways not foreseen or taken into account during the design, certification, training, and procedure development for these highly automated airplanes. Prior to the advent of reliable and highly capable automation, the typical pilot response to an abnormal situation (e.g., an equipment malfunction or an unexpected event) would have been to turn the automation off and fly the airplane manually. As the automation became more capable and reliable, it became easier and potentially safer to handle some of these situations with the assistance of the automation (e.g., one-engine-inoperative driftdown from cruise altitude, one-engine-inoperative approach or go-around). Other situations (e.g., an unexpected response from the autoflight system) were handled by either turning the automation off or reverting to a lower level of automation.

More recently, there have been situations where flightcrews have either inappropriately continued to use the automation when they found themselves in an abnormal situation or, if the automation was initially off, turned the automation on to try to accomplish a recovery. Examples include:

- Fixation on following the flight director and ignoring airplane attitude. In one particular case, this resulted in a low speed excursion, after which the flightcrew engaged the autopilot to accomplish the recovery.

- Using the autopilot to recover from an overspeed warning rather than resorting to manual control.

- Attempts by the flightcrew to engage the autopilot in the moments preceding the March, 1995, crash of a Tarom A310 at Bucharest as they attempted to recover from an extreme bank angle resulting from a large thrust asymmetry. 
- Engagement of the autopilot by the flightcrew of the A300-600 that crashed at Nagoya, Japan in April, 1994 -- apparently in response to difficulties in maintaining the glide slope following the inadvertent activation of the takeoff/go-around levers.

These types of actions are contrary to current widely held assumptions about pilot behavior that are used in designing, evaluating, training, and operating highly automated airplanes. These assumptions are based on a certain level of basic airmanship, which plays an important role in how the flightcrew interacts with automation. The unexpected pilot behavior evidenced in recent accidents and incidents appears to be the result of many factors, including the increased capability, reliability, and authority of the automated systems, increased flightcrew use of and reliance on such systems, protective features of these systems (real or imagined), automation philosophy (or lack thereof) of the operator, and cultural differences. An additional factor may be that flightcrews are becoming less confident in their own airmanship skills relative to the capabilities they perceive to be present in the automation, particularly in a stressful situation. In some cases, where this perception of the automation's capabilities is particularly inaccurate, it can have potentially hazardous consequences. For example, contrary to the belief of many flightcrews, some autoflight systems will take the airplane outside of the normal flight envelope (e.g., speed below stall warning speed or above the maximum operating limit speed), or attempt maneuvers that would not be expected of a human pilot. These characteristics can have potentially hazardous consequences, especially if the flightcrew is unaware of them.

Unexpected flightcrew actions and changing patterns of flightcrew behavior have implications for the design and evaluation of automated systems. During the design process, designers must make assumptions about the range of behaviors expected of the pilots who will use these systems. Regulatory officials evaluate the designs, again making assumptions about expected pilot behavior. These assumptions appear to be in need of reassessment in light of recent experience.

Degraded or inadequate situation awareness can also influence the flightcrew's decisions regarding the level or mode of automation to use. Confusion over what level of automation has been selected or is actually engaged, or inadequate understanding of the airplane's flight path relative to potential safety threats can lead to inappropriate automation use. These points are further developed in the "Flightcrew Situation Awareness" section of this report.

The HF Team also received several comments regarding mixed-mode flying. Mixed-mode flying combines elements of automatic and manual control such that the airplane is neither completely under automatic control, nor is it solely under manual control (e.g., manually controlling pitch, bank, yaw, and flight path while the autothrottle is engaged). Some operators expressly discourage mixed-mode flying on some airplane types, while others generally encourage its use as a means to retain manual skills proficiency while minimizing workload and taking advantage of partial task automation (e.g., using the autothrottle to maintain speed control). Possible hazards of mixed-mode flying are that it can lead to unintended mode changes or configurations, cause cross-coupling and inappropriate pitch 
or thrust responses, mask trends in the airplane's flight path or energy state, or make it more difficult to discern who (or what) is controlling the airplane. The HF Team considers mixed-mode flying to be appropriate when conducted in a manner consistent with the airplane manufacturer's design intent and assumptions. However, flightcrews should be trained in its advantages, limitations, and proper use. Also, specific procedures should be established and included in training programs. Where mixed-mode flying is not recommended due to potential vulnerabilities, operators should carefully adhere to the manufacturer's procedures or constraints.

The HF Team notes that several operators have recently established a clearly enunciated philosophy regarding automation use and distributed it to their flightcrews. The HF Team supports this practice and believes it to be a valuable foundation for promoting flightcrew understanding of operator policies, procedures, and practices regarding automation use.

\section{Flightcrew Non-Adherence to Procedure}

According to one study of accident prevention strategies, 8 "pilot flying adherence to procedure" could have potentially prevented more accidents than any other single strategy examined. However, the study did not attempt to address the reasons why flightcrews deviate from procedures, nor does it consider the number of accidents or incidents that may have been prevented because the flightcrews deviated from procedures. Also, current methods of assessing system safety do not provide a means to measure the success or failure of any particular accident prevention strategy.

The development of standard operating procedures and flightcrew understanding and adherence to these procedures is an important part of the defense against hazards resulting from flightcrew error. This point is well known; however, the HF Team found weaknesses in several areas relative to current practices for developing and implementing standard operating procedures. Due to the strong link between procedural deficiencies and airplane accidents, the HF Team considers it important to address this issue. The HF Team is particularly concerned about the following types of procedures:

- Procedures used by operators that are inconsistent or conflict with the airplane manufacturer's design philosophy and recommended procedures (e.g., not using autobrakes, flight directors, or other systems/features as designed);

- Procedures that are used as work-arounds for design deficiencies (e.g., flightcrew callout of mode changes as a primary means for providing mode awareness; forbidding programming the FMS below a certain altitude);

- Procedures that are not covered adequately in training (e.g., use of FMS vertical flight path modes);

- Procedures or procedural steps that do not promote understanding of the action(s) that the flightcrew are to undertake, especially for procedural items that do not appear to be directly related to the desired objective (e.g., consequences of activating or not

${ }^{8}$ Accident Prevention Strategies. Boeing Commercial Airplane Group, October, 1993. 
activating the approach mode on certain FMS systems and the use of FMS oneengine-inoperative driftdown procedures);

- Incomplete consideration of the potential for errors and the resulting hazards, especially when using the procedures under varying circumstances (e.g., inappropriate use of the open descent mode at low altitude, changing FMS arrival runway information, and inadvertent deletion of intermediate route or altitude constraints); and

- Procedures carried over from one airplane type to another for standardization, but could have unintended consequences or are otherwise inappropriate for the different airplane type (e.g., not using autobrake capability for rejected takeoffs or not using flight director information when it is readily available and suitable for the task).

\section{Recommendations}

\section{Recommendation AutomationMgt-1:}

The FAA should ensure that a uniform set of information regarding the manufacturers' and operators' automation philosophies is explicitly conveyed to flightcrews.

\section{Discussion of Recommendation AutomationMgt-1:}

The information provided to flightcrews should include:

- The manufacturer's higher level design philosophy (e.g., the reasons for automating particular functions) to the extent that this philosophy could affect operational use;

- The operator's automation philosophy, which should be used as the basis for operator policies, procedures, and practices related to automation use;

- The principles of operation (e.g., operating assumptions used in the design, such as the basis for the computation of vertical flight profiles);

- A description of the envelope protection features, including specific capabilities and limitations, and the situations or flight conditions for which envelope protection is or is not available; and

- Guidance (including rationale) relative to selecting the appropriate level of automation for routine use and for non-routine situations (e.g., when confused by automation response, engine failure in different phases of flight, unusual attitudes, speed excursions (high or low), terrain or collision avoidance, flight path deviations, or unexpected or difficult air traffic clearances or requests).

The operator's automation philosophy should be consistent with the overall design philosophy and principles of operation. Because of differences among manufacturer's 
automation philosophies (and sometimes among airplane types from the same manufacturer), operator's automation philosophies may need to be differentiated by airplane type or significant variant. Standard operating procedures should be consistent with the operator's automation philosophy for each airplane type and should promote understanding of the action(s) expected of the flightcrew and the automation. When developing the operating procedures, consideration should be given to potential sources of error under varying circumstances.

\section{Recommendation AutomationMgt-2}

The FAA should require operators' manuals and initial/recurrent qualification programs to provide clear and concise guidance on:

- Examples of circumstances in which the autopilot should be engaged, disengaged, or used in a mode with greater or lesser authority;

- The conditions under which the autopilot or autothrottle will or will not engage, will disengage, or will revert to another mode; and

- Appropriate combinations of automatic and manual flight path control (e.g., autothrottle engaged with the autopilot off).

Discussion of Recommendation AutomationMgt-2:

Most of this information may be available in current training and operating manuals; however, it is typically scattered throughout several volumes and may not be emphasized to the extent necessary for flightcrews to grasp its practical significance. Current qualification programs may cover this material to some extent, but it is generally not emphasized to the extent the HF Team considers necessary, nor is it integrated with training, simulator, or Line Oriented Flight Training (LOFT) scenarios. The HF Team recommends consolidating this information into clear and concise guidance to promote better flightcrew understanding of the capabilities and limitations of the automation, and, to the extent necessary, incorporating practical demonstrations of its use into training and checking scenarios or events. This recommendation is not intended to encourage rote responses to specific situations, but rather to demonstrate practical cases where safety can be improved by appropriate automation choices.

\section{Recommendation AutomationMgt-3}

The FAA should initiate a review of the autopilots on all transport category airplanes to identify the potential for producing hazardous energy states, excessive pitch or bank angles, subtle departures from the intended flight path, slow-overs, hard-overs, or other undesirable maneuvers. Results of this review should be the basis for initiating appropriate actions, such as design improvements, flight manual revisions, additional operating limitations, or changes in training programs or operational procedures. 
Discussion of Recommendation AutomationMgt-3:

The HF Team considers this review to be necessary due to evidence that assumptions of pilot behavior used in the design and evaluation of autopilots in the current fleet of transport category airplanes do not appear to adequately cover the range of pilot behaviors being encountered in service. Unexpected pilot behavior, coupled with inconsistent autopilot protective features, can and have resulted in unsafe situations. These vulnerabilities should be identified and appropriate action taken to ensure continued operational safety. Although the HF Team examined this issue to some extent, we lacked the resources and expertise to accomplish a thorough review.

This review should be conducted in a cooperative effort with representatives from the FAA Aircraft Certification and Flight Standards Services, airplane manufacturers, avionics manufacturers, and operators. The participation of representatives from the Joint Aviation Authorities (JAA) is also strongly recommended. (See also related Recommendations AutomationMgt-2, SA-1, and SA-2.)

\section{Recommendation AutomationMgt-4}

The FAA should assure that analyses are conducted to better understand why flightcrews deviate from procedures, especially when the procedural deviation contributes to causing or preventing an accident or incident.

Discussion of Recommendation AutomationMgt-4:

In order to fully understand the role of procedural deviations as a contributory factor in accidents and incidents, it is important to determine why the flightcrew deviated from procedures. Simply listing flightcrew procedural deviations as a contributory factor, without determining whether there were more fundamental reasons for the procedural deviations, inappropriately implies that exhorting flightcrews to always follow procedures will prevent these accidents or incidents. In the presence of more systemic problems, such a strategy is destined to fail. The system must be improved, and to do that, one must identify and understand the deficiencies in the system. For example, did the flightcrew deviate from procedures because the procedures were too difficult to understand, had unintended consequences, did not fit the situation, were too ambiguous or contradictory, or because they were incomplete? Or was it that the flightcrew was complacent or used bad judgment because they lacked certain knowledge or skills? Are there features of the flight deck design or the flightcrew interfaces that lead to procedural deviations, either alone or in combination with the recommended procedures?

To find effective ways to prevent procedural deviations from contributing to future accidents and incidents, the HF Team recommends that the aviation community thoroughly assess and understand the reasons behind these deviations. Within the 
limitations of the available data, cases should be studied where procedural deviations prevented or otherwise had a beneficial effect on the outcome of an accident or serious incident.

The HF Team notes that some studies have begun in this area (e.g., by a subcommittee of the ATA Human Factors Task Force) and we support these efforts.

This recommendation is related to Recommendation Measures-2, and the implementation of these two recommendations should be coordinated.

\section{Recommendation AutomationMgt-5}

\section{The FAA should request industry to take the lead in developing design guidelines for the next generation of flight management systems.}

Discussion of Recommendation AutomationMgt-5:

The HF Team identified concerns regarding current FMS designs such as the following:

- The need for standardization of route, leg, and constraint conventions such as waypoint entry conventions, definition, and implementation of vertical profiles (e.g., vertical navigation (VNAV)), etc. to reduce error potential and facilitate easier transitioning between airplane types or derivatives;

- Critical or irrevocable entries should be confirmed before they are executed, as well as providing an "undo" capability when appropriate;

- Response time should be improved when long response times can lead to flightcrew distraction from other essential tasks or cause programming errors;

- Titles of pages and relationships among different pages should be clear and unambiguous so as to facilitate easy access to information;

- Unanticipated dropping of information (e.g., waypoint, altitude constraints) should be addressed when it leads to frequent incorrect path definition or excessive workload in using "workarounds;" and

- Error messages should be meaningful and helpful (e.g., in response to improper entry) and assist the flightcrew in correcting the entry (e.g., "invalid entry" is insufficient, instead provide the appropriate format to use or identify the missing information).

Due to a variety of considerations, manufacturers may be reluctant to change designs that have been in use for years. Updating the FMS interface will require a major commitment by both industry and government and may need to be tied to additional Communication, Navigation, and Surveillance (CNS) or Air Traffic Services (ATS) benefits. The cost of developing, validating, and verifying the software for redesign of such a system is cited as one of the major reasons for maintaining the current general design. However, cooperation 
between the regulatory authorities and industry, with flexibility shown by all parties, can result in a better, more human-centered design, as well as achieving more effective CNS capabilities and operator benefits. 
This page intentionally left blank 


\section{Flightcrew Situation Awareness}

"Needless to say, confusion was in abundance. There are just too many different functions that can control airspeed and descent rates, all of which can control the altitude capture."

"My first priority was data entry rather than situational awareness."

"Had he continued to follow the flight director, we would have had a full power stall in IFR conditions...I believe we are slowly working ourselves into detrimental reliance on FMS/glass cockpits/autoflight systems."

"We missed the crossing altitude by 1000 feet. The captain was...busy trying to program the FMC. Being new in an automated cockpit, I find that pilots are spending too much time playing with the computer at critical times rather than flying the aircraft. No one looks outside for traffic."

- Quotes from the ASRS database

Situation awareness is a widely used term, but its meaning often varies depending on the context in which it is being used. In the context of this report, we use this term to refer to the flightcrew knowing and understanding the present and future status of the airplane and its systems, based on the airplane's state and flight path parameters (e.g., the airplane's position, speed, flight path, energy state, and position of the flight controls) and the status and behavior of the autoflight system relative to the operating environment (e.g., terrain, air traffic clearances, and other traffic). Inadequate assessment, understanding, or monitoring of any of these parameters contributes to deficiencies in situation awareness, and may lead to inappropriate flightcrew actions.

The introduction of the electronic horizontal situation indicator and the navigation display in glass cockpit airplanes has increased flightcrews' ability to maintain lateral, and to a lesser extent, vertical situation awareness. These displays are capable of displaying the airplane's current and future horizontal flight path superimposed on an electronic map. The quality of information provided on these displays, however, depends on how well the flightcrew sets up and manages the display(s). For example, if inappropriate range scales are selected, or necessary navigational information is not properly set up or selected, the advantages of the electronic map display may be negated. Also, in some cases, the very compelling nature of these displays may be leading to complacency and a deterioration in basic position awareness skills. In circumstances where the electronic map is unavailable, or the display has not been properly configured by the flightcrew, there is a potentially a greater vulnerability to a degradation in position awareness than previously existed. 
The HF Team has concerns that incidents demonstrating deficiencies in flightcrew monitoring and awareness of autoflight system modes, airplane energy state, terrain proximity, and airplane systems' status are occurring to an unacceptable extent. An incomplete understanding of flightcrew feedback needs, inadequate integration of warnings and alerts, variation in automation interfaces among different airplanes, and flightcrew non-adherence to procedures also contribute to vulnerabilities in current highly automated airplanes. The HF Team has also identified several specific hazardous states of awareness that are indicative of a loss of flightcrew vigilance or alertness. These concerns are discussed in detail below.

The HF Team emphasizes that these concerns extend beyond deficiencies in crews' monitoring techniques and adherence to procedures. Design and training aspects that influence the ability of flightcrews to maintain situation awareness must also be addressed.

\section{Autoflight System Mode Awareness}

Actions and responses of any autoflight system vary depending on what autoflight mode(s) is active. Being aware of the active mode(s) and understanding the corresponding actions and responses is necessary for proper use of the autoflight system. During the course of this study, the HF Team identified several factors that inhibit crews' awareness, knowledge, and understanding of autoflight system modes:

- Salience of the mode annunciations. Flightcrews must read and interpret from a variety of alphanumeric symbols (e.g., VNAV PATH, VNAV ALT, ALT**, G/S, LOC, THR HOLD, SPD), some of which are present for only a brief period of time, to determine which mode(s) is active or what mode change has occurred. (See Figure 2 for examples of mode annunciation symbology.) For most of the airplane types under study, the mode annunciations appear on the primary flight display (PFD). This information competes for the flightcrew's attention with the generally more conspicuous graphical displays of attitude, speed, and altitude information that also appear on the PFD. Mode changes can easily be missed, even when additional cues are provided (e.g., drawing a box around the new mode and/or using a flashing display for a few seconds), unless a flightcrew member is looking at the display when the change occurs. With the autopilot on, flightcrew members are often not looking at the PFD when a mode transition occurs. In addition, the meaning behind current mode annunciations can be ambiguous because the same mode annunciation may represent a different airplane state or behavior in different situations. Accordingly, having the information available is sometimes insufficient; it must also be salient and unambiguous. 


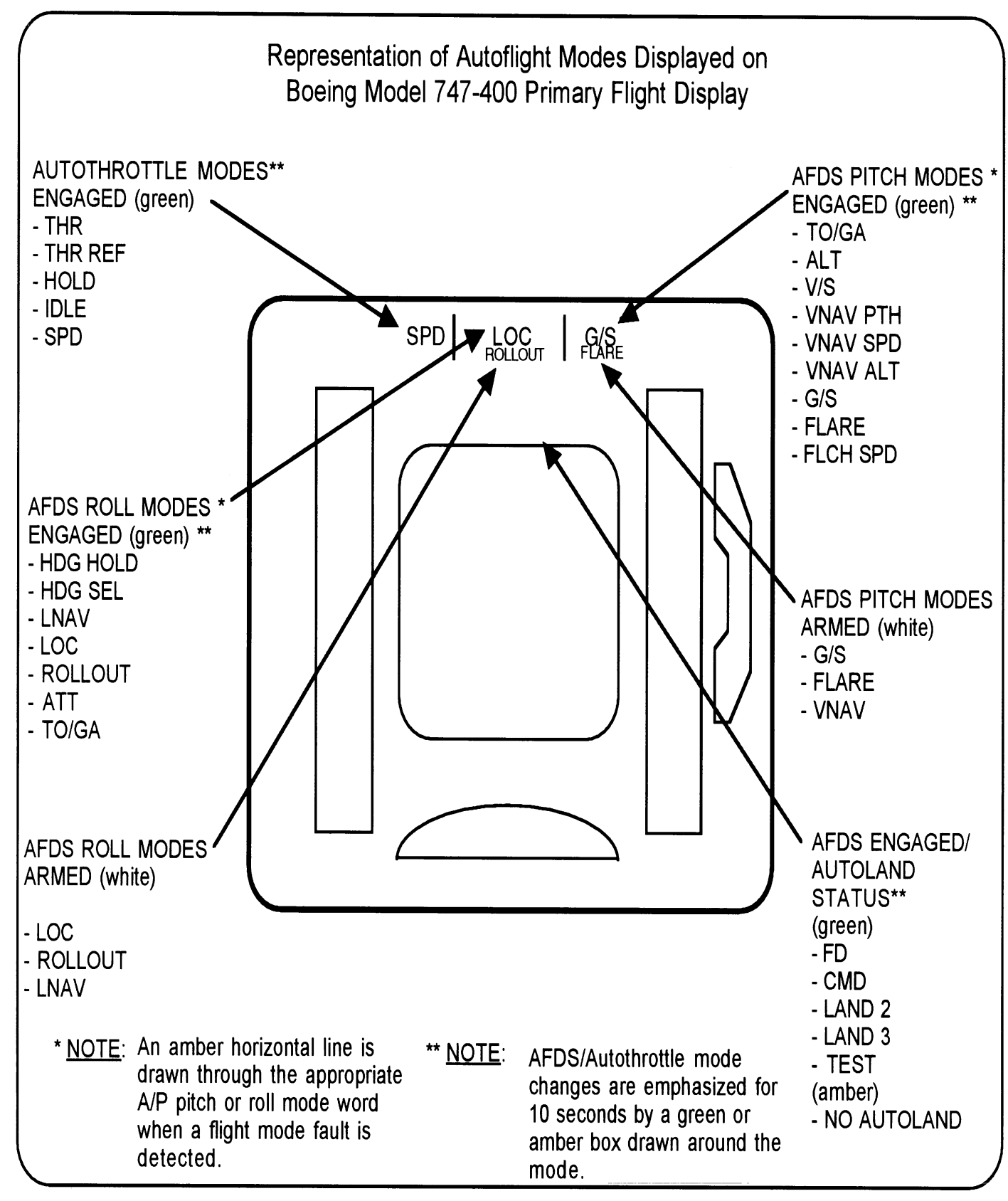

Figure 2

- Methods for monitoring mode information. Standard instrument scan patterns used with older analog instruments may not apply to glass cockpit displays. The HF Team notes that nothing comparable to the standard instrument scan pattern has arisen for these new displays, especially in terms of continuously monitoring mode information. Instead, there are conflicting ideas on how best to maintain awareness of the active mode. For example, some manufacturers and operators recommend that flightcrews call out all mode changes. Other manufacturers and operators find this philosophy too 
burdensome and consider it to be unnecessary and potentially distracting, especially for mode changes that are associated with normal system behavior.

- Indirect mode changes. Mode changes that are not due to a direct flightcrew action are more likely to go unnoticed or create confusion. These indirect mode changes may be the result of previously programmed instructions, an exceedance of the design or flight envelope parameters for the current mode, or they may represent transition states between modes selected by the flightcrew. Because indirect mode changes do not involve either flightcrew input or confirmation at the time of the mode change, flightcrews may be unaware that a mode change has occurred. The mode change may result in significant differences between the flightcrew's expectations and the airplane's actual behavior. An indirect mode change played a role in the September, 1994 airplane stall incident involving a Tarom Airbus A310-300 over Paris-Orly. In that incident, an indirect mode change occurred as a result of an overshoot of the flap placard limit airspeed.

An example of a similar type of mode change on Boeing airplanes is the transition from a vertical navigation path mode to a vertical navigation speed mode when an airspeed tolerance value is exceeded while on the programmed vertical flight path. Only a subtle change in the mode annunciation (the annunciator changes from VNAV PTH to VNAV SPD) informs the flightcrew that the airplane will not fly the commanded profile and will probably not meet the next and possibly subsequent programmed altitude/airspeed constraints.

\section{- Differences in mode nomenclature and display among different airplane types.} Modes intended to accomplish a similar objective may have different names and use different nomenclature for the flightcrew interface. For example, the "open descent" mode on Airbus A320 airplanes performs a very similar function to the "flight level change" on Boeing, Douglas, Fokker, and some other Airbus airplanes. Despite the different nomenclature, these modes operate in basically the same way -- thrust is held constant at a pre-determined value while the autopilot supplies pitch commands to the elevator to fly the commanded airspeed.

In some airplanes, the vertical navigation modes used in connection with the flight management system are referred to as "VNAV." In other airplanes, these modes are called "profile" (PROF) or "managed navigation." Boxes around mode annunciations may mean one thing on some airplanes, and something different on other airplanes. Even the name of the panel on which the mode selectors are located differs from manufacturer to manufacturer. Airbus calls it a flight control unit (FCU), Boeing a mode control panel, Douglas a flight control panel, and Fokker a flight mode panel.

The arrangement of mode annunciations also differs markedly between airplanes. In some airplanes, (like the 747-400 example shown in Figure 2), the current modes are shown in three fields arranged horizontally across the top of the PFD. On other 
airplanes, the mode information is divided into four or five fields and may appear either on the PFD or on a separate flight mode annunciator display, while on still others this information is displayed vertically in the lower left and right corners of the PFD. Not only does the location or number of fields of information differ, but these differences also reflect different philosophies for how the information should be grouped. For example, one philosophy would be to inform the flightcrew what is controlling the airplane's speed, and the lateral and vertical aspects of the flight path. Another philosophy groups the information in terms of the autothrottle, pitch, and heading modes.

These examples are but a few of the many nomenclature, configuration, and display differences between different airplanes that exist throughout the autoflight system. While the safety implications of these differences can be difficult to determine, at the minimum such differences can be confusing to flightcrews moving from one airplane type to another and impose an additional training burden on operators. In non-normal circumstances, a pilot's instinctive reaction, if developed on a different airplane type than the one currently being flown, can lead to an incorrect action.

- Differences in the design implementation of modes that are intended to accomplish the same objective. For example, one airplane may fly a linear path between two altitude constraints, while a different airplane may remain at the altitude of the first constraint until it can fly an idle thrust descent to the second constraint. These differences in design implementation occur not only between airplane manufacturers, but also occur on different airplane types from the same manufacturer. Subtle differences in the way the modes work are not only confusing to flightcrews and air traffic controllers, but also have significant implications for the design of operational procedures and air traffic clearances, since different airplane types may fly different flight paths.

- Proliferation in the number of modes. There was broad consensus among those with whom the HF Team met that there are simply too many different modes, many of which perform similar functions. For example, vertical speed, flight level change, VNAV path, VNAV speed, and Flight Path Angle (FPA) are all different modes that can be used during a descent. Figure 2 shows the number of different modes available on a Boeing 747-400 (and is also representative of other highly automated airplanes). The large number of modes increases the training burden placed on operators and pilots and increases the complexity of the interface, leading to increased risks of flightcrew error.

Reducing the number of modes, however, would not be an easy task. To a large extent, the proliferation of modes is due to the varied needs of the operating environment, different operators, and different operating procedures. It is not that any one operator needs, or even wants, all of these modes. Quite the contrary, the input received by the HF Team indicates that none of the operators who responded to this issue uses or needs every mode that has been provided. However, when considering in 
total the varied requests of different operators, each individual mode is deemed necessary or desirable by one or more operators.

One suggestion offered to the HF Team to reduce the number of modes would remove the annunciation of certain transition states (e.g., altitude capture), since these states are not directly selectable by the flightcrew, do not require further flightcrew action, and are only active for a short period of time. The HF Team disagrees with this approach. The HF Team considers it important to keep the flightcrew informed of any automation state that may result in a change in the airplane's or automation's behavior, or could result in a different response to flightcrew actions.

- Complexity in the flightcrew interface (as perceived by the flightcrew), especially for the vertical modes. We heard many complaints about the non-intuitiveness of existing interface designs. Flightcrews noted that elegant engineering solutions do not necessarily produce user-friendly designs. Of particular concern are the vertical modes because they give flightcrews the most difficulty.

Complexity in the flightcrew interfaces carries the price of increasing the potential for flightcrew error. This potential is increased when actions taken by the autoflight system differ from the actions the flightcrew would take. Remedies are usually obtained through training, standard operating procedures, warnings, alerts, etc. However, these remedies can only go so far -- more attention needs to be paid to this problem in the design of the interface.

- Conflicting information provided by the control panel used for selecting autoflight modes. On some airplane types, push-button mode selectors illuminate or otherwise show they have been selected regardless of whether or not the selected mode is actually engaged. Although flightcrews are trained to refer to the mode annunciators (usually located on the PFD) to identify active modes, some flightcrews look to the mode selector panel for this information, and are vulnerable to receiving incorrect feedback. The distance between the selector knobs and buttons to the PFD annunciators contributes to this tendency for pilots to use the mode selection indicators to provide feedback on the active modes.

\section{Airplane Energy State, Terrain, and Systems Status Awareness}

\section{Airplane Energy State Awareness}

Based on a review of numerous incident and accident reports, the HF Team is concerned that flightcrews may not be provided adequate awareness of airplane energy state, particularly when approaching or trending toward a low energy state. The incorporation of features such as autotrimming, attitude rate or maneuver demand flight control laws, and autopilot modes such as control wheel steering and vertical speed can make it more difficult for the flightcrew to recognize conditions that may lead to low energy states. 
Transport category airplanes are required to have adequate warnings of an impending stall, but at this point the airplane may already be in a potentially hazardous low energy state. Better awareness is needed of energy state trends such that flightcrews are alerted prior to reaching a potentially hazardous low energy state.

\section{Terrain Awareness}

Although the introduction of Ground Proximity Warning Systems (GPWS) in the 1970's greatly reduced the number of controlled flight into terrain (CFIT) accidents, CFIT accidents continue to occur at an unacceptable rate. A high percentage of these accidents occurs in the landing configuration during low visibility non-precision approaches. Also, nuisance warnings and delayed or nonexistent warnings continue to degrade the utility of current warning systems.

The HF Team believes that the improved navigation and communication capabilities of advanced automated airplanes offer the potential for significantly improving the safety of some approaches, especially those into difficult airports lacking ground facilities to provide vertical approach guidance. Operators such as Alaska Airlines are demonstrating the capabilities of FMS lateral and vertical path guidance using required navigation performance (RNP) area navigation procedures at locations such as Juneau, Alaska. American, Delta, and Northwest Airlines are also demonstrating the safety and efficiency of FMS lateral and vertical path guidance at airports such as Eagle, Colorado. The HF Team believes changes can and should be made to current departure and approach procedures where possible to take full advantage of existing airplane and infrastructure capabilities. For the future, increased emphasis should be given to developing and implementing entirely new concepts for aiding flightcrew terrain awareness.

\section{Systems Status Awareness}

In general, the incorporation of electronic system synoptic and warning displays has increased flightcrews' ability to evaluate the status and activity of airplane systems and equipment. However, the HF Team has several concerns with the implementation of these displays on modern transport category airplanes. First, there is a lack of standardization within the industry regarding display symbology, nomenclature, and content. Second, in some airplanes, the complexity and variety of ancillary warnings and alerts associated with major system failures can make it difficult for the flightcrew to discern the primary failure. For example, following certain engine failure events, alerts associated with the enginedriven subsystems (e.g., hydraulic, pneumatic, electric, fuel) may mask the primary failure or distract the flightcrew, making it more difficult to recognize the principle cause (e.g., engine failure). Third, on some airplanes, discrete indications of systems status have been completely eliminated such that the flightcrew must rely solely on the electronic display's warning messages to diagnose a problem. Last, there is a tendency to provide binary state indications (OK or not $\mathrm{OK}$ ) for some parameters, rather than a continuous display of parameter values. Some of these issues are less of a concern on the more recent airplane 
types, and the HF Team hopes to see a continuation of the trend toward including better system failure diagnostic tools.

\section{Variations in the Automation Interfaces Among Different Airplane Types}

In addition to the issue of different nomenclature for essentially the same function, which is discussed above for mode awareness, there is a lack of standardization for basic features such as data entry conventions and display symbology, the location of takeoff/go-around and autothrottle disconnect switches, and the layout of the autoflight system mode selector panel. Examples of the wide variations in the layout of the mode selector panel are shown in Figure 3. Figure 4 provides examples of variations in the location of takeoff/go-around switches and autothrottle disconnect switches, and Figure 5 shows some of the many variations in formatting conventions for navigation position data.

There are also different conventions employed for selecting or engaging modes from the mode selector panel. Pushing, pulling, or twisting the selector knobs or buttons will achieve different results on different airplanes. The vulnerability resulting from all these variations is that flightcrews transitioning between airplane types may find habits and previous training difficult to overcome. Further, the chance for error is increased, especially during stressful situations.

The HF Team is especially concerned about the use of multi-function knobs for flight critical functions and the use of different autoflight controls that have a similar shape, feel, location, and display (e.g., speed and heading control knobs). These design features are contrary to the principles of minimizing the potential for flightcrew error and providing error tolerance. These features make it too easy for a busy flightcrew member to make an error and not realize it until the airplane's behavior becomes sufficiently different from what the flightcrew expects. For example, it is believed by some that the similarity between the display representations of flight path angle and vertical speed played a major role in the Air Inter Airbus A320 accident at Strasbourg, France in 1992, and in several similar incidents.

\section{Warning and Alerting Schemes}

A multitude of warnings and alerts exist in the cockpits of many modern transport category airplanes to notify the flightcrew of potentially hazardous situations. A variety of methods are employed to take advantage of most of the human senses to get the flightcrew's attention, including voice, horns, klaxons, chimes, bells, cavalry charges, buzzers, wailers, clackers, alphanumeric messages, blinking lights, flashing displays, stick shakers, different colors, etc. Many of these warnings have been mandated as a result of safety issues brought to light by specific incidents or accidents. 
Figure 3. Mode Selector Panels

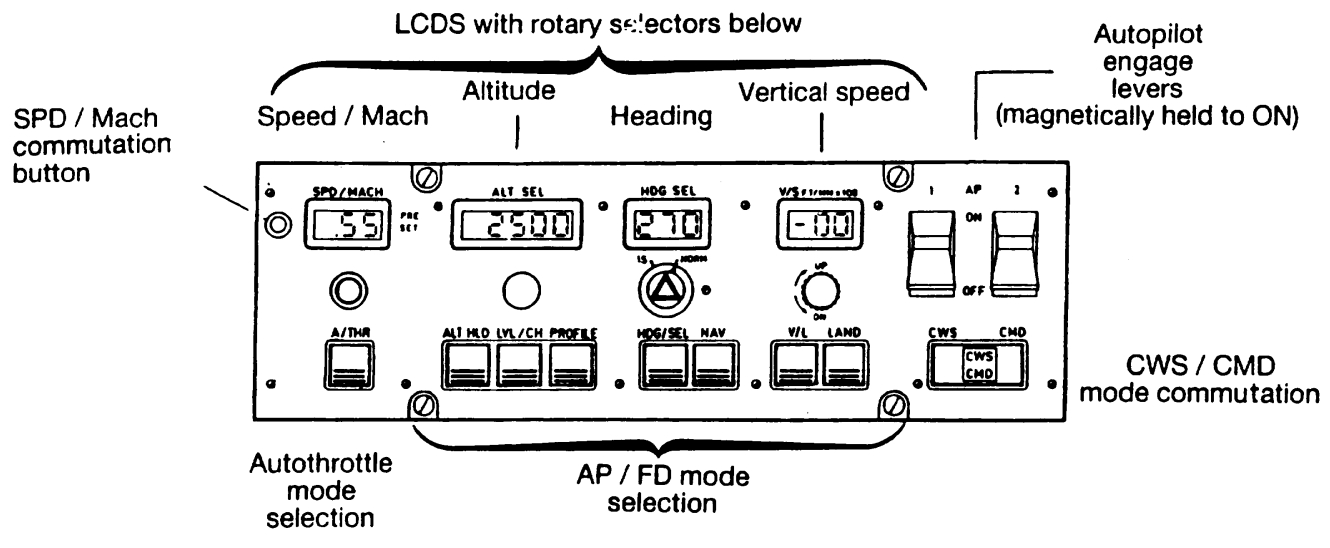

Airbus A310

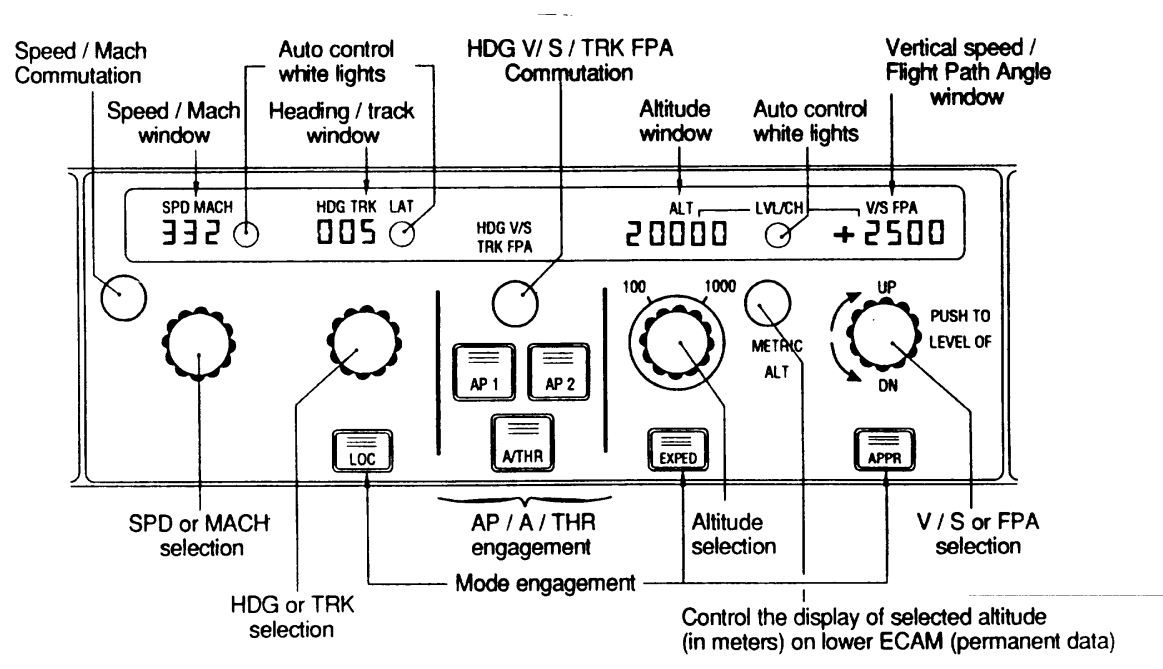

Airbus A320

(essentially the same for A330/A340) 
Figure 3 (continued)

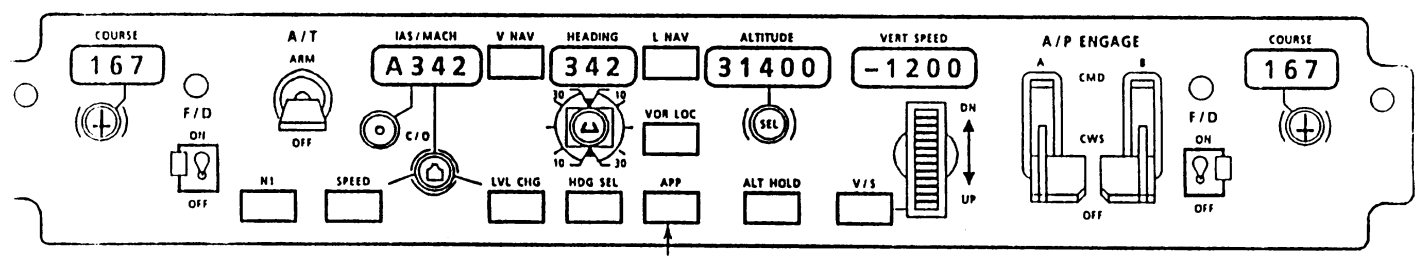

Boeing 737-300/400/500

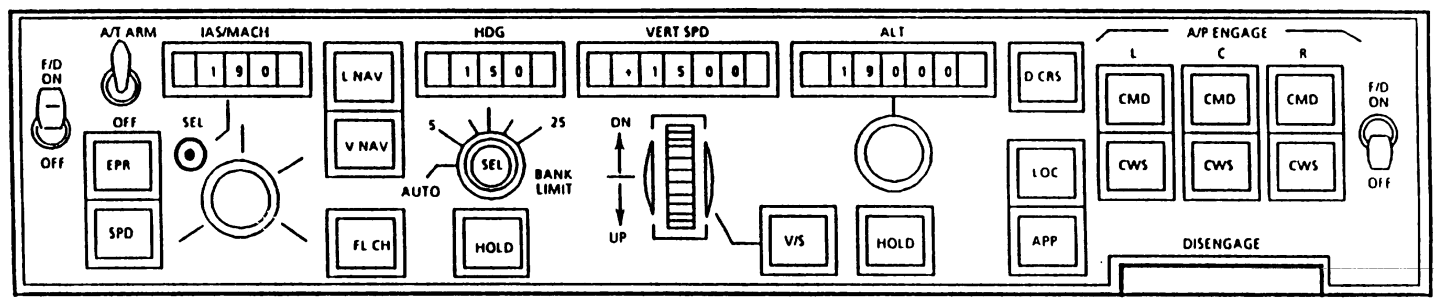

Boeing 757/767

(also Boeing 747-400 with deletion of the four push button selectors associated with the backcourse (B/CRS) and control wheel steering (CWS) options)

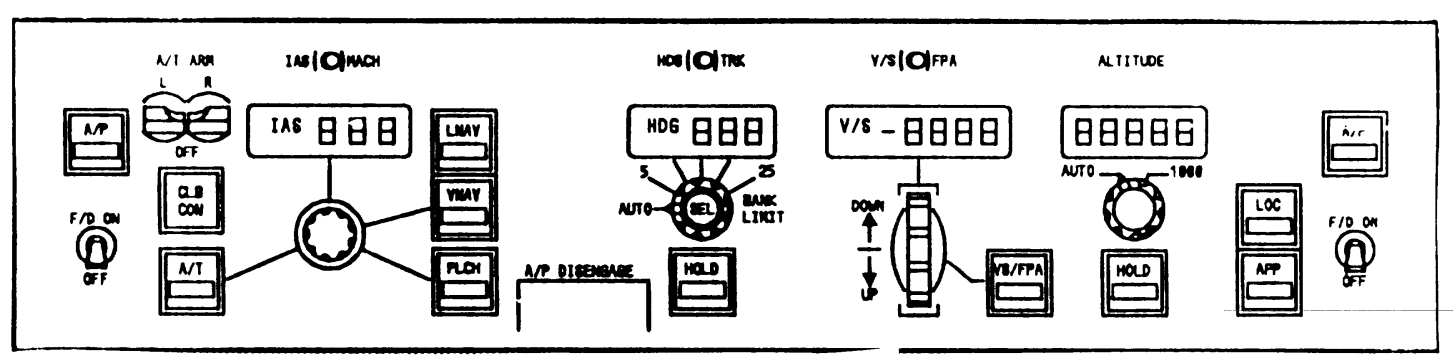

Boeing 777 
Figure 3 (continued)

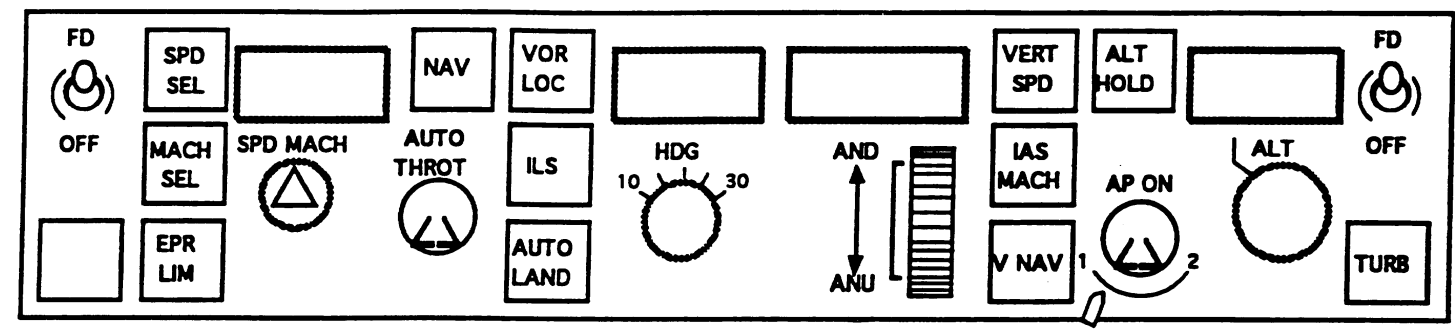

Douglas MD-80/MD-90

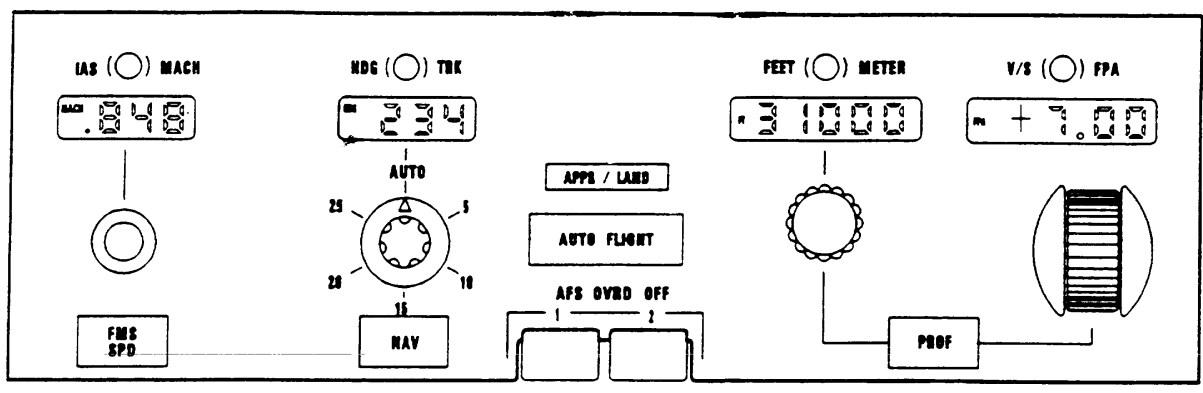

Douglas MD-11

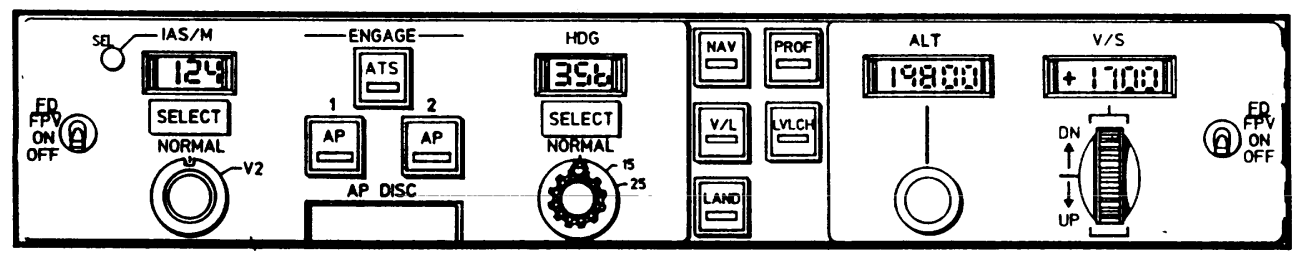

Fokker F28-0100 (F100) 


\section{Figure 4. Takeoff/Go-Around and Autothrottle Quick}

\section{Disconnect Switch Locations}

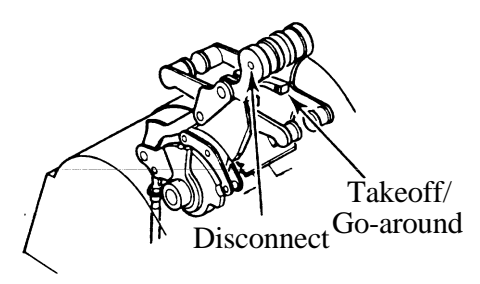

A300-600

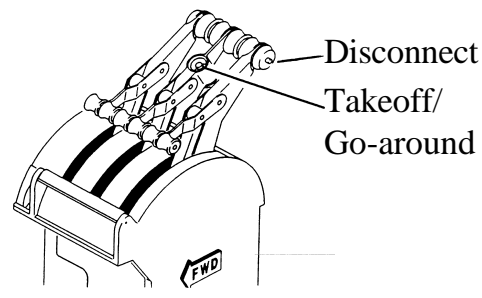

MD-11

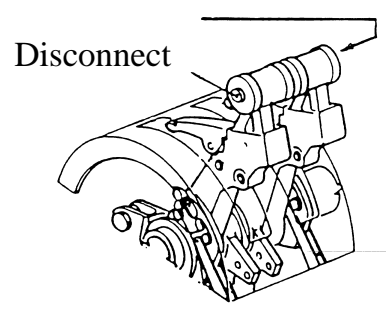

A320

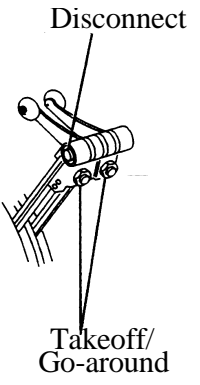

MD-90

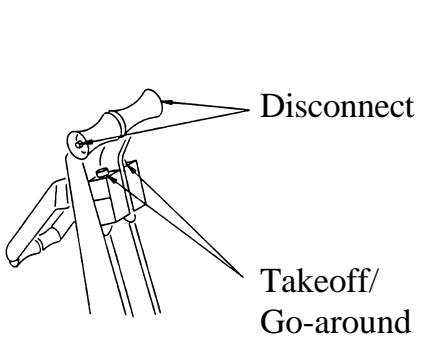

737

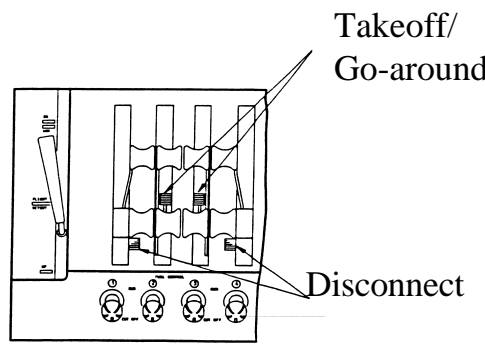

$747-400$
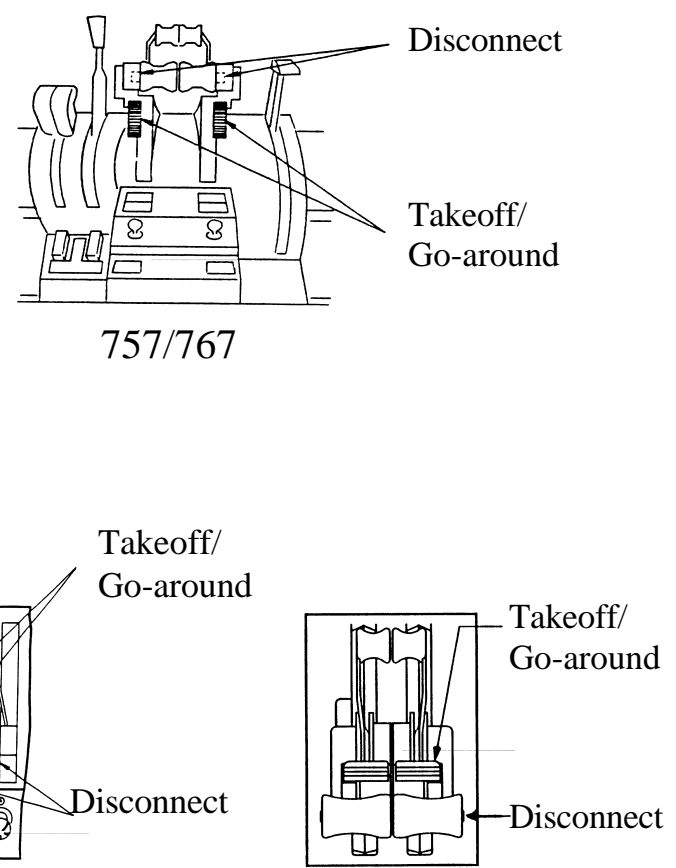

777 
Figure 5. Sample of Various Formatting Conventions

For a Given Geographic Fix

FAA Source Data

(FAA Order 8260.19/7100.11)

FAA to National Flight Data Center

(Ref. FAA Order 7900.2)

U.S. Govt Flt Info Pub Supplement

Aeronautical charts: Jeppesen
5000 00.00/030 0000.00 (deg-min-sec) $500000.00 \mathrm{E} / 0300000.00 \mathrm{~S}$ (if not $\mathrm{N}$ or W)

$50^{\circ} 00^{\prime} 00.00^{\prime \prime} \mathrm{N} 30^{\circ} 00^{\prime} 00.00^{\prime \prime} \mathrm{W}$

N5000.00’W03000.00'

N50 00.0 W030 00.0

- or -

N50 00.00

W030 00.0

$\mathrm{N} 50^{\circ} 00.0$

$\mathrm{W} 030^{\circ} 00.0^{\prime}$

$50^{\circ} 00^{\prime} \mathrm{N}-30^{\circ} 00^{\prime} \mathrm{W}$

(Airport Reference Point)

Arinc 424 specification:

N50 000000 W030 000000

Full FMS Conventions:

N5000.0W03000.0

N50W030

Abbreviated FMS entry format

$5030 \mathrm{~N}$

(Waypoint formats for 5-character unnamed reporting points)

Arinc Communications Addressing

50N030W

and Reporting System entry format

Flight Deck Communications

N50 W030

\section{- Position report}


Although there has been much progress made in integrating, prioritizing, and, when appropriate, inhibiting unnecessary alerts, the HF Team is concerned both that the number and complexity of warnings and alerts has grown too large and that existing warnings and alerts may not always be integrated into a consistent scheme. Multiple warnings and alerts may also mutually interfere or may interfere with flightcrew communication at critical times. Contributing to this problem are FAA regulatory standards that mandate the means by which a specific warning or alert must be implemented, regardless of whether it fits in with the warning or alerting philosophy adopted by the manufacturer. Examples of mandated warning systems that require distinctively different warnings include landing gear, takeoff configuration, overspeed, stall, Traffic Alert and Collision Avoidance System (TCAS), GPWS, and the predictive and reactive windshear alerting systems.

The more unique warnings there are, the more difficult it is for the flightcrew to remember what each one signifies. The result can be a confused and distracted flightcrew precisely at the time when prompt action may be necessary. Inappropriate use of color, sound, etc. may also cause confusion, as may several warnings and alerts going off in unison and perhaps conflicting with one another (e.g., the flightcrew of the Birgenair Boeing 757 that crashed into the sea shortly after takeoff from Puerto Plata, Dominican Republic may have been confused by conflicting stall and overspeed warnings coupled with erroneous airspeed information). Increasing levels of automation coupled with the evolving operational environment (e.g., Data link, the Future Air Navigation System, free flight) and new safety systems (e.g., predictive windshear and enhanced GPWS) make it more critical then ever that advisories, alerts, warnings, and status information be properly integrated.

\section{Feedback Needs}

Empirical research, incidents, and accidents suggest that flightcrews tend to detect unexpected automation behavior in these highly automated airplanes from observations of unanticipated airplane behavior (e.g., speed or flight path deviations or unexpected movement of a control) rather than from displays containing information on automation status/configuration. ${ }^{9}$ Since the information needed by the flightcrew to detect the undesired automation behavior is already available on cockpit displays, this observation suggests that current feedback mechanisms may be inadequate to support timely error detection.

Several incidents and accidents point to other vulnerabilities that are associated with the autoflight system masking system failures or other causes of in-flight upsets. These vulnerabilities result when the autoflight system initially masks the in-flight upset, then suddenly disengages or is unable to maintain control when it runs out of control authority. Because of the masking effect of the autopilot, these situations may not be adequately

\footnotetext{
${ }^{9}$ Sarter, Nadine B. and David D. Woods. 'How in the world did we ever get into that mode?' Mode Error and Awareness in Supervisory Control. Human Factors, 37(1), 5-19, 1995.
} 
addressed by the current autopilot regulatory requirements. Examples that illustrate these vulnerabilities include:

- A China Airlines Boeing 747 in 1985 lost power on one engine during cruise in autoflight. The captain was unaware of the engine failure, in part because the autopilot compensated for the resulting yaw until control limits were reached. Upon disengagement of the autopilot, the resulting transient caused a rapid roll and steep dive angle. The captain was able to successfully regain control of the airplane.

- An American Eagle Aerospatiale ATR-72 crashed near Roselawn, Indiana in 1994 after a severe icing encounter. The autopilot disconnected shortly after the ailerons deflected, initiating an abrupt roll to the right that the flightcrew was unable to arrest.

- A number of high altitude upset incidents have occurred on the Airbus A300-600 in which FMS performance data indicated an altitude capability very near the buffet limit. When turbulence was encountered, the autopilot would disconnect, leaving the flightcrew with an airplane out of trim, near buffet, and with marginal stability. Serious turbulence or flight control-induced "airplane-pilot coupling" incidents have also been encountered on the Douglas MD-11, involving a fatality in one instance. These incidents appear to be exacerbated by high altitude stability characteristics, flightcrew unfamiliarity with these characteristics, and autopilot interactions.

The type of feedback provided to the flightcrew is changing with the evolving technology in both the flight deck interface and the flight control systems. In many areas, tactile feedback is being replaced by visual annunciations. Although the same information may be present, its form has changed. One particular example of this change is illustrated by the use of non-moving autothrottles in Airbus A320/A330/A340 airplanes. In these airplanes the thrust levers do not move in response to changes in thrust commanded by the autothrust system. The tactile cues present in other airplanes (which Airbus suggests may be misleading because the thrust lever position is only an indication of the commanded thrust level) are replaced by additional visual cues (e.g., flight mode annunciations, a speed trend symbol on the PFD, and enhanced presentation of engine parameters) augmented by envelope protection features and aural alerts (on some airplanes) for low energy state. ${ }^{10}$

Another example of a change in the type of feedback provided in the A320/A330/A340 airplanes is the use of uncoupled sidesticks, which do not provide direct tactile feedback of a pilot's control stick inputs to the other pilot, nor feedback as to the position or movement of the flight control surfaces. Because the uncoupled sidesticks make it more difficult to for flightcrews to discern the other pilot's inputs (and there have been cases of inadvertent conflicting flightcrew inputs), there are additional flightcrew coordination issues to address. It is difficult to determine whether the changes in the type of feedback

\footnotetext{
${ }^{10}$ For a discussion of the potential benefits and disadvantages of non-moving autothrottles, refer to SAE Technical Paper Series, number 912225, British Airways Airbus A320 Pilots' Autothrust Survey, by Steve Last and Martin Alder; and National Aerospace Laboratory of the Netherlands, NLR TP 94005, Pilot Performance in Automated Cockpits: A Comparison of Moving and Non-Moving Thrust Levers, by H.H. Folkerts and P.G.A.M. Jorna.
} 
associated with the non-moving autothrottles and uncoupled sidesticks meet (or do not meet) the pilot's needs, however, because of a lack of understanding and consensus of precisely what type and amount of feedback are necessary.

Despite the amount of interest paid to the specific design features noted above, there are other more generic examples of the changing nature of feedback associated with highly automated airplanes that also need further attention. Just as in the examples cited above, however, there is a lack of consensus on their relative importance and potential effects. Nonetheless, one example is the increased use of the visual channel to sense the present and future flight path through display annunciations rather than the tactile sensations of the movements of the control column and thrust levers. We also heard from several operators that the use of autoflight systems has increased the need for verbal communications between flightcrew members, because it can be more difficult for pilots to discern the inputs and the intentions of the other pilot. Often, these inputs affect the future flight path of the airplane rather than the current flight path, and the delay between the action and the effect raises coordination issues. Another example is provided by the trend in modern autopilot design to use very gradual flight path changes for improved passenger comfort. The situation may be further aggravated by quiet flight decks, where auditory cues (e.g., those associated with engine thrust changes) are not as noticeable.

Other automation issues, such as flightcrew complacency and over-reliance on automation, should also be considered in examining flightcrew feedback needs. An inflight upset of a Boeing 747 operated by Evergreen International Airlines in 1991 (and other similar incidents) highlighted the vulnerabilities related to these issues when accompanied by a hard-to-detect automation failure. In the 1991 incident, an autopilot failure caused a departure from the desired flight path in the form of a slow roll that was below the threshold for flightcrew perception. Outside visual references were also unavailable. The flightcrew first became aware of the resulting flight path deviation and excessive bank angle when the inertial navigation system FAIL lights illuminated. They then noted that the instruments indicated a bank angle in excess of 90 degrees.

The HF Team concluded that there is a lack of credible data and consensus regarding what constitutes effective feedback and how best to provide it. We found strongly held, but differing opinions regarding the proper balance between visual, aural, and tactile feedback under different situations. Additional work needs to be done to understand and objectively evaluate flightcrew feedback needs.

\section{Hazardous States of Awareness}

Inattention, or decreased vigilance, is often cited in ASRS reports, and has been a contributor to operational errors, incidents, and accidents. Decreased vigilance manifests itself in several ways, which can be referred to as hazardous states of awareness. These states include: 
- Absorption. Absorption is a state of being so focused on a specific task that other tasks are disregarded. Programming the FMS to the exclusion of other tasks, such as monitoring other instruments, would be an example of absorption. The potential for absorption is one reason why some operators discourage their flightcrews from programming the FMS during certain flight phases or conditions (e.g., altitudes below 10,000 feet).

- Fixation. Fixation is a state of being locked onto one task or one view of a situation even as evidence accumulates that attention is necessary elsewhere or that the particular view is incorrect. The "tunneling" that can occur during stressful situations is an example of fixation. For example, a pilot may be convinced that a high, unstabilized approach to landing is salvageable even when other flightcrew members, air traffic control, and cockpit instruments strongly suggest that the approach cannot be completed within acceptable parameters. The fixated pilot will typically be unaware of these other inputs and appear to be unresponsive until the fixation is broken. Fixation is difficult to self-diagnose, but it may be recognizable in someone else.

- Preoccupation. Preoccupation is a state where one's attention is elsewhere (e.g., daydreaming).

Decreased vigilance can be caused or fostered by a number of factors, including:

- Fatigue. Fatigue has been the subject of extensive research and is well recognized as a cause of decreased vigilance.

- Underload. Underload is increasingly being recognized as a concern. Sustained attention is difficult to maintain when workload is very low.

- Complacency. Automated systems have become very reliable and perform most tasks extremely well. As a result, flightcrews increasingly rely on the automation. Although high system reliability is desired, this high reliability affects flightcrew monitoring strategies in a potentially troublesome way. When a failure occurs or when the automation behavior violates expectations, the flightcrew may miss the failure, misunderstand the situation, or take longer to assess the information and respond appropriately. In other words, over-reliance on automation can breed complacency, which hampers the flightcrew's ability to recognize a failure or unexpected automation behavior. 


\section{Recommendations}

\section{Recommendation SA-1}

The FAA should require operators to increase flightcrews' understanding of and sensitivity to maintaining situation awareness, particularly:

- Mode and airplane energy awareness issues associated with autoflight systems (i.e., autopilot, autothrottle, flight management system, and fly-by-wire flight control systems);

- Position awareness with respect to the intended flight path and proximity to terrain, obstacles, or traffic; and

- Potential causes, flightcrew detection, and recovery from hazardous pitch or bank angle upsets while under autopilot control (e.g., wake vortex, subtle autopilot failures, engine failure in cruise, atmospheric turbulence).

\section{Discussion of Recommendation SA-1:}

This recommendation is intended as a near-term temporary solution until these issues are more comprehensively addressed in design, flightcrew qualification/training, and operational procedures. In coordination with the FAA, airplane manufacturers and operators should develop and issue additional guidance emphasizing the importance of maintaining situation awareness in highly automated airplanes. This guidance should include discussion and examples of monitoring techniques and the potential hazards associated with inadequate monitoring or understanding of autoflight modes, airplane energy state, position and flight path, and the potential causes and characteristics of inflight upsets that may initially be masked or otherwise exacerbated by the autoflight system. Examples should be provided of problems encountered in incidents, accidents, inservice difficulties, and training. Examples of items that flightcrews should be made aware of include:

(1) The lack of low speed protection features in many autopilots when in any vertical mode;

(2) Situations in which uncommanded or indirect mode changes may occur, and the implications of those mode changes; and

(3) Situations that can result in hazardously low energy states when using the control wheel steering autopilot mode on airplanes with a conventional control system or during manual flight of airplanes with a fly-by-wire control system when the particular implementation of these systems results in neutral longitudinal speed stability (i.e., stick force versus speed). 
The information provided should take into account the clarity and completeness of existing flightcrew operating manual (FCOM) information available to flightcrews and should emphasize critical FCOM information related to major areas of vulnerability (as determined from incidents, accidents, etc.). This information should be provided through existing methods for increasing flightcrews' awareness of specific safety issues. Examples include manufacturers' publications (operator letters, bulletins, and periodicals) or special topic training aids (e.g., Windshear Training Aid, Takeoff Safety Training Aid), operators' publications (safety bulletins, newsletters), and regulatory agency advisories (e.g., FAA Flight Standards Information Bulletins, Handbook bulletins).

\section{Recommendation SA-2}

The FAA should require operators' initial and recurrent training programs as well as appropriate operating manuals to:

- Explicitly address autoflight mode and airplane energy awareness hazards;

- Provide information on the characteristics and principles of the autoflight system's design that have operational safety consequences; and

- Provide training to proficiency of the flight management system capabilities to be used in operations.

Discussion of Recommendation SA-2:

This is a follow-on recommendation to Recommendation SA-1 and is intended to address the same issues on a longer term basis. (See also Recommendation Knowledge-2.)

Operators should be required to incorporate the information developed in response to Recommendation SA-1 into their initial and recurrent training programs as well as into appropriate operating manuals. In addition, the HF Team considers it important for operators and flightcrews to understand the manufacturer's underlying design principles for the automation, including both higher level philosophy (e.g., the reasons for automating a particular function) and lower level principles and characteristics that have operational safety consequences (e.g., the basis for the computation of vertical flight profiles or one-engine-inoperative driftdown profiles). Operating procedures should, as appropriate, be consistent with the underlying automation design principles.

Flightcrews should be given sufficient training on using the FMS to ensure proficiency at least for those capabilities used in normal day-to-day operations. The HF Team considers the practice of expecting flightcrews to acquire these basic skills while flying the line to be inappropriate. 


\section{Recommendation SA-3}

The FAA should encourage the aviation industry to develop and implement new concepts to provide better terrain awareness.

Discussion of Recommendation SA-3:

Continued vulnerabilities to controlled-flight-into-terrain accidents demonstrate the need for further improvement in this area. The objective of this recommendation is to encourage timely development of better defenses against this class of accidents. New approaches are needed to supplement or replace the current ground proximity warning systems, such that earlier indications and warnings of potential collisions with terrain are provided and nuisance warnings are minimized.

A potential approach currently being proposed uses terrain databases in conjunction with accurate position information (e.g., from the global navigation satellite system), prediction algorithms for the airplane's future flight path, graphical terrain depiction on an electronic display, and suitable flightcrew alerting. The HF Team supports this approach, but candidate proposals should be carefully evaluated to ensure proper integration with other flight deck systems and displays, and that human performance issues and other potential hazards (e.g., errors in terrain databases) are satisfactorily addressed.

\section{Recommendation SA-4}

The FAA and the aviation industry should develop and implement a plan to transition to standardized instrument approaches using lateral navigation (LNAV) and vertical navigation (VNAV) path guidance for three-dimensional approaches. The use of approaches that lack vertical path guidance should be minimized and eventually eliminated.

\section{Discussion of Recommendation SA-4:}

This recommendation is intended to reduce the vulnerability to controlled-flight-intoterrain accidents, especially those associated with approaches lacking suitable approach guidance. To accomplish the goal of this recommendation, a suitable existing or newly established working group should be tasked to recommend an implementation plan and schedule to the FAA. The working group should include at least representation from FAA Aircraft Certification, Flight Standards, and Air Traffic Services, operators, airplane and avionics manufacturers, pilots, and other affected parties. 


\section{Recommendation SA-5}

The FAA should encourage the exploration, development, and testing of new ideas and approaches for providing effective feedback to the flightcrew to support error detection and improved situation awareness.

\section{Discussion of Recommendation SA-5:}

The FAA should encourage, either through research, technical committees, or other collaborative processes, the development of new approaches, tools, and criteria for improving feedback in the cockpits of highly automated airplanes under normal, abnormal, and emergency conditions. This effort should address:

- Flightcrew information needs;

- How to provide better feedback of airplane energy state trends;

- Issues related to the value of specific types of feedback (e.g., when is tactile feedback necessary?); such as

- Whether, and under what circumstances, one feedback channel can be substituted for another (e.g., visual for tactile);

- Overloading of feedback channels (e.g., guidance on the maximum acceptable number of discrete auditory alerts);

- How automation can potentially mask situations that may develop into problems;

- Changes in flightcrew information needs and feedback effectiveness in going from normal to abnormal to emergency conditions (e.g., investigate issues such as display de-cluttering, integration of warnings and alerts);

- Masking of abnormal situations by the autoflight system;

- Improved methods of presenting vertical flight path information to the flightcrew;

- How to reveal transitions across modes (show events, targets, and indirect mode transitions);

- How to show the future airplane behavior (reveal what should happen next and when);

- How to reveal patterns (pilots should be able to scan at a glance and pick up possible unexpected or abnormal conditions, rather than have to read and integrate each individual piece of data to make an overall assessment);

- How to provide flightcrews with feedback to help them understand the behavior of autoflight systems, especially with respect to vertical navigation (i.e., what it is doing now and what it is going to do in the future); and 
- How to provide better feedback on the activities of the autoflight system, particularly when:

(1) The autoflight system takes an action not explicitly directed by the flightcrew (e.g., a mode reversion);

(2) The autoflight system overrides, denies, or otherwise inhibits an action commanded by the flightcrew; and

(3) The autoflight system is about to take an action of interest to the flightcrew.

The process should include prototyping, testing, and widespread adoption of successful innovations to aid awareness and monitoring, where better error detection is one criterion for success. From this effort, the FAA should pursue internationally harmonized guidelines for incorporating more effective feedback mechanisms related to both present and future operating environments.

\section{Recommendation SA-6}

The FAA should encourage standardization, as appropriate, of automation interface features, such as:

- The location, shape, and direction of movement for takeoff/go-around and autothrottle quick disconnect switches;

- Autoflight system mode selectors and selector panel layout;

- Autoflight system modes, display symbology, and nomenclature; and

- Flight management system interfaces, data entry conventions, and nomenclature.

Discussion of Recommendation SA-6:

The FAA should encourage appropriate standardization of automation interface features by supporting recently initiated efforts in industry technical committees and exploring incentives for standardization (and possibly disincentives for inappropriate differentiation) that would lead or assist in the development of guidelines and standards. These guidelines and standards should also address the use of multi-function controls and differentiation of controls by location, shape, and feel.

Standardization is not intended to substitute for human-centered design, but implemented correctly, it can reduce the potential for flightcrew error. It can also reduce the training burden for transitioning flightcrews and improve the reliability of proper human response, particularly when reacting instinctively in critical situations. One potential pitfall of standardization that should be avoided is to standardize on the lowest common denominator (e.g., disabling the autobrakes on airplanes that have this feature because it is not included on all airplane types). Another potential pitfall is that inappropriate 
standardization, rigidly applied, can be a barrier to innovation, product improvement, and product differentiation. In implementing this recommendation, these potential pitfalls should be recognized and avoided. It may be appropriate to interpret this recommendation as a request for consistency, rather than rigid standardization.

\section{Recommendation SA-7}

The FAA and the aviation industry should update or develop new standards and evaluation criteria for information presented to the flightcrew by flight deck displays and aural advisories (e.g., primary flight displays, navigation/communication displays, synoptics showing system states).

\section{Discussion of Recommendation SA-7:}

The objective of this recommendation is to encourage the industry to adopt standard methods of displaying information to the flightcrew on electronic displays (e.g., speed and altitude tape displays, map symbols, attitude information for unusual attitudes, traffic displays, systems displays). Consideration should also be given to new communication/navigation system elements to be incorporated into cockpits, such as data link, RNP, and enhanced GPWS.

Feedback issues associated with implementation of this recommendation should be addressed in a coordinated manner with the effort recommended in Recommendation SA-5.

\section{Recommendation SA-8}

The FAA should ensure that flightcrews are educated about hazardous states of awareness and the need for countermeasures to maintain vigilance. The FAA should encourage operators to:

- Develop operational procedures and strategies to foster attention management skills with the objective of avoiding hazardous states of awareness; and

- Develop techniques to apply during training to identify and minimize hazardous states of awareness.

Discussion of Recommendation SA-8:

This recommendation is intended to be a near term means of addressing the issues associated with hazardous states of awareness. Existing knowledge regarding states of awareness and attention management skills should be used to educate operators and to facilitate development of the training techniques and operational procedures and strategies referred to in the recommendation. For example, Crew Resource Management (CRM) training could include methods for recognizing hazardous states of awareness in other 
flightcrew members (e.g., fixation, absorption) as well as methods for combating these states.

\section{Recommendation SA-9}

The FAA should sponsor research, or assure that research is accomplished, to develop improved methods for:

- Evaluating designs for susceptibility to hazardous states of awareness (e.g., underload, complacency, absorption); and

- Training to minimize hazardous states of awareness.

Discussion of Recommendation SA-9:

This recommendation addresses the hazardous states of awareness issue from a longer term perspective than Recommendation SA-8. Further research on the issue should be sponsored by the FAA to develop criteria, tools, and methods for use in designing systems that minimize susceptibility to hazardous states of awareness, evaluating the success of these designs, and for developing training techniques or system designs that recognize and minimize these states. 


\section{Communication and Coordination}

The HF Team examined several areas within the aviation system where there is insufficient communication and coordination that can affect the safe operation of highly automated airplanes. Insufficient communication and coordination have led to incompatibilities between the capabilities of highly automated airplanes and the air traffic service environment, and has inhibited the sharing of in-service data to identify vulnerabilities before they result in an incident/accident. Both inter- and intra-organizational communication difficulties within the FAA can impede both FAA and industry personnel from performing their respective roles in a consistent and ideal manner. Lack of coordination has also resulted in or contributed to a proliferation of technical committees dealing with identical (or nearly identical) issues, and research that is either incomplete or does not get applied.

\section{Incompatibility Between Airplane Capabilities and the Air Traffic Service Environment}

"I do not believe that ATC controllers understand the operation of computer driven aircraft..."

"Controllers need to understand the increase in workload that is placed on a 2-man crew using an FMC when giving restrictions and holding instructions... We are plagued with late clearances, frequent changes..."

"Simple changes to [ATC] procedures would help cut out workload so we could keep our heads out of the cockpit and still use the computer..."

- Quotes from the ASRS database

In many ways, advanced cockpit automation has greatly added to the flightcrew's ability to operate safely within the confines of the air traffic environment. Complex departure and approach paths, altitude constraints, en route navigation, etc. can be pre-programmed, reducing flightcrew workload and making it easier, for the most part, to conform to air traffic clearances. Certain features have been added, such as the electronic horizontal situation indicator (i.e., "moving map" display), that assist the flightcrew in visualizing and understanding the implications of these clearances.

We were provided with numerous examples, however, that provide evidence of incompatibilities between highly automated airplanes and the air traffic service environment. In the HF Team's discussions with airplane operators, pilot groups, and 
airplane and avionics manufacturers, there is broad consensus that these incompatibilities represent a significant issue impacting the safety and efficiency of current operations. For example, late changes to approach and landing clearances can create potentially unsafe high workload situations for the flightcrews of highly automated airplanes as they attempt to reprogram the revised arrival information. Or flightcrews may be forced to revert to lower levels of automation, thereby negating any advantages that may have been available through use of the full automation capabilities.

As another example, some established approaches and departures are either incompatible with highly automated airplanes or do not allow optimal use of the automation. "Slamdunk" approaches, which involve high rates of descent in the last stages of the approach, present problems for any airplane, highly automated or not. For example, approaching San Francisco International Airport, it is not uncommon to be held at altitudes over 7000 feet on the downwind leg, then be requested to turn onto the final approach leg, and land with very little distance in which to accomplish the descent. The HF Team believes that such procedures need to be carefully reviewed in order to provide the proper balance between safety and capacity issues.

In general, problem areas fall into one of three classifications:

(1) Clearances that present difficulties for any airplane, but are particularly difficult for highly automated airplanes, such as:

- Flight paths near the limit of the airplane's performance capability (e.g., "slam dunk" approaches);

- Last minute changes in identifying the runway to use for takeoff or landing; and

- Late clearances for higher (or lower) altitudes during climb (or descent) or for crossing constraints.

(2) Clearances that were developed for and based on the capabilities of older airplanes, and may be difficult to perform using the advanced cockpit automation, such as:

- Tracking outbound on a Very High Frequency Omnidirectional Radio Range (VOR) radial;

- Back course approaches;

- Tuning and listening to the Automatic Terminal Information Service (ATIS) in the rare instance when the ATIS frequency coincides with an Instrument Landing System or VOR frequency rather than a communication frequency; and

- A go-around with an altitude, heading, or flight track that is complex and differs from the published missed approach. 
(3) Clearances that do not take advantage of the unique capabilities of FMS-equipped airplanes, such as:

- Headings to intercept radials at an arbitrary point or flight over radio navigational aids versus direct routings to a waypoint;

- Fuel- and airspace-inefficient climb and descent trajectories versus VNAV trajectories that permit efficient, coordinated arrival and departure paths; and

- Non-precision approach concepts with numerous step down fixes versus three-dimensional LNAV/VNAV arrivals and departures that provide vertical path guidance, and LNAV/VNAV approaches to a runway end rather than to arbitrary points away from the landing flight path.

An additional concern with certain air traffic procedures has arisen recently with the development of highly accurate navigation information (e.g., the Global Positioning System (GPS)). For example, some procedures that may have provided appropriate separation between airplanes on intersecting arrival and departure paths may no longer be appropriate in a GPS environment. The high degree of precision provided by modern navigation systems using GPS may actually increase the chance of collision if there is a procedural failure or human failure in the use of these procedures. Examples include the use of a common fix for arrival and departure procedures where aircraft are pointed directly at each other during climb and descent, or oceanic tracks where aircraft are assigned clearances to fly exactly the same oceanic track for long distances. These air traffic procedures and routes should be re-evaluated and modified or eliminated.

Incompatibilities between airplane capabilities and the air traffic services environment have resulted in inappropriate altitude, speed, and heading assignments, increased controller and flightcrew workload, degradation of flightcrew situation awareness, and inefficient use of fuel and airspace. Additionally, air traffic procedure demands, if not well coordinated with the users of the air traffic system and the airplane manufacturers, add undue complexity to airplane autoflight system designs, operational procedures, and training because of the variety of procedures that are developed without regard to airplane system design consequences. Resolving this issue presents significant challenges on the national level, but will be even more difficult when the international variation in air traffic systems is considered.

Nevertheless, these concerns must be addressed. Early implementation of new CNS and air traffic management concepts (e.g., increased use of direct routings, RNP, and free flight), both in the U.S. National Airspace System and internationally, can play an important role in resolving some of these incompatibility issues.

\section{Insufficient Communication About In-Service Experience}

The aviation industry has an enviable overall safety record. In achieving this safety record, the risk of accidents due to "simple" types of failures (e.g., equipment, mechanical, or 
structural failures from which recovery is impossible) has been greatly reduced in the current generation of transport category airplanes. Most of the accidents now result from a number of factors converging in a particular way (i.e., an "accident chain" ${ }^{11}$ ). Because accidents are so infrequent, accident data are insufficient to provide an adequate source of information for making further safety improvements. (See the section entitled, "Measurement of and Incentives for Safety," for further discussion of this point.)

Errors, incidents, and other in-service events provide vital data that can also be used to prevent future accidents. As can be seen from the list of incidents and accidents provided in Appendix D, many of the accidents examined by the HF Team were preceded by incidents involving similar circumstances. As one example, three years prior to the China Airlines A300-600 accident at Nagoya, Japan, an A310 was involved in a serious incident of a similar nature. As another example, the A320 accident at Strasbourg, France was preceded by several incidents that pointed to the possibility for confusion over the similarity between the vertical speed and flight path angle annunciations.

During the HF Team's discussions with the various segments of the industry, strong concerns were voiced about the lack of communication of in-service events data. Although some sharing of data takes place, and there are systematic data collection systems that deal with a portion of the available information (e.g., ASRS, British Airways' Safety Information System), there is a need for a system-wide process for collecting, analyzing, and reporting data to appropriate parties. The Department of Transportation and the FAA have recognized this need and, with support from the aviation industry, have outlined steps toward accomplishing this goal. ${ }^{12}$ The HF Team endorses this approach, but notes that timeliness is critical and that the resulting process must be designed to adequately address human performance issues.

The HF Team also noted that information on difficulties encountered in operational service or in training that could affect flight safety is not systematically being passed on to flightcrews. Flightcrews may also be unaware of the particular circumstances involved in relevant accidents and major incidents. The HF Team considers it especially important that flightcrews be made aware of this type of information since, as end users in this system, they are very important links in the safety chain. As an example, prior to the 1993 landing accident of a Lufthansa Airbus A320 at Warsaw, it was not widely recognized that when landing with flaps "Full," there are certain conditions in which the spoilers may not deploy on landing, even if the pilot manually moves the speedbrake control to the deploy position.

Another example is a subtle and not widely known characteristic of the rudder throw limiter on the Douglas MD-80. Following an engine failure or other thrust asymmetry, it may be necessary for the pilot to first relax full rudder pressure, momentarily center the rudder pedals, and then reapply full rudder pedal deflection in order to gain full rudder travel authority. The consequences of this characteristic for some engine failure scenarios were not widely known by MD-80 pilots, and may still not be known to pilots at some

${ }^{11}$ Accident Prevention Strategies. Boeing Commercial Airplane Group, October, 1993.

${ }^{12}$ Aviation Safety Plan. U.S. Department of Transportation, February, 1996. 
operators. Nevertheless, it was reported to be a factor in an incident at one U.S. airline. That airline subsequently incorporated information about this characteristic into manuals and procedures. It is unknown how many other operators have addressed this issue. This is an example of the type of information that should be widely and quickly shared between operators, manufacturers, pilots, and regulatory authorities to help prevent recurrence of potentially avoidable incidents and accidents.

In our meetings with operators, the operators noted that manufacturers frequently requested data from them, but did not always share data that was in the manufacturer's possession. Operators are often told that the particular problem they reported was unique, implying that the operator may have been at fault when the problem may actually have been a generic one. In turn, manufacturers claimed a paucity of data from operators. Without sufficient data from the operators, manufacturers cannot identify and fix problems.

The FAA requires air carriers and manufacturers to report equipment failures, malfunctions, and defects, but does not require other types of events to be reported. In some areas, FAA reporting requirements are very outdated. For example, current requirements do not specifically address difficulties experienced in the flightcrew/automation interface. As another consideration, FAA enforcement responsibilities often inhibit the collection and sharing of a large and important segment of in-service data, particularly as it relates to flightcrew performance. Liability concerns are another inhibiting factor.

\section{Deficiencies in Information Provided to Flightcrews in Charts, Approach Plates, Instrument Procedures, Meteorological Data, and Notices to Airmen (NOTAM)}

Information provided to flightcrews in charts, approach plates, instrument procedures, meteorological data, and NOTAMs is sometimes difficult to read and understand, and the information is not presented in a prioritized manner. Difficulties in reading and understanding the charts may have been a contributory factor in the December, 1995 accident of an American Airlines Boeing 757 near Cali, Columbia.

The HF Team found that NOTAMs are perceived as being particularly difficult to read and understand. Pilots must often look in several different locations to find the relevant information, abbreviations and terms, etc. As a result, important information can easily be missed. Moreover, the system is inconsistent internationally, and is not well suited to the needs of flightcrews of highly automated airplanes.

\section{Communication and Coordination Deficiencies Within the FAA}

The FAA is staffed with highly skilled and dedicated employees. However, the HF Team found that links between FAA organizations are sometimes too weak, such that many FAA personnel are unable to take full advantage of expertise outside of their own 
organization. For example, many Aircraft Certification and Flight Standards Services personnel rarely have contact with other FAA offices with which they should be routinely communicating. Also, difficulties were observed in the coordination of FAA research activities with the needs of FAA certification or operations specialists, and in the dissemination of research results to the appropriate specialists.

Due to the make-up of the HF Team, Team members have first-hand experience of communication and coordination difficulties between the Aircraft Certification and Flight Standards Services. Certification personnel may be unaware of some of the particulars of the operational environment, including the capabilities and limitations of current line pilots and the environment in which they operate. Flight Standards personnel may be unaware of airworthiness certification requirements or the assumptions about the operational environment made during certification. Therefore, it is extremely important for specialists from these organizations to constantly interact with each other. Inadequate communication and coordination between these groups can result in inconsistencies between the airworthiness assumptions made during certification and the operational suitability of a product in service.

Good communication and coordination are especially difficult for supplemental type certification projects when the project's Aircraft Certification Office (ACO) does not have an Aircraft Evaluation Group (AEG) associated with it, or ACO personnel are not used to working with an AEG. Another area in need of improvement is the lack of formal involvement by appropriate ACO personnel in the Flight Operations Evaluation Board (FOEB) and the Flight Standardization Board (FSB). The FOEB's principal task is to develop the Master Minimum Equipment List, which addresses the acceptability of operations with inoperative equipment. The FSB sets flightcrew qualification standards. Participation by relevant specialists from the ACO is necessary to identify and explain the assumptions made during the type certification approval process. Currently, participation of ACO representatives in the FOEB and FSB is infrequent due primarily to a lack of resources. Similarly, the involvement of AEG personnel in airworthiness certification efforts is often resource-limited.

The HF Team also found deficiencies in communication and coordination within and between other FAA organizations. Too often, FAA offices operate independently of each other, providing different levels of service and interpretive guidance to applicants. The Directorate system has helped to remedy this situation to some extent, but it has not eliminated the problem. Applicants continue to complain about uneven treatment by different FAA offices, and the HF Team was provided similar comments.

\section{Coordination of Technical Committees}

There are too many technical committees working independently on the same, or very similar, issues with little coordination between them. These groups often have some differences in their charters, but also have many common interests. In many instances, these groups fail to communicate with each other on common issues. For example, flight 
management system issues are being discussed by the Airlines Electronic Engineering Committee, an Air Transport Association of America (ATA) task force, the Radio Technical Commission for Aeronautics, the International Civil Aviation Organization (ICAO), and three Society of Automotive Engineers (SAE) committees (S-7, G-10, and G-13). As another example, within SAE alone, there are three separate committees (G-10, A-4, and S-7) working on Head-up displays. None of these committees are coordinating with the FAA/JAA all-weather operations harmonization effort, which is an important customer for SAE's efforts. Limited regulatory and industry resources make it difficult to support this proliferation of independent committees dealing with common or related issues.

\section{Coordination and Communication Between Research Community and End Users}

The research community has devoted significant attention to human factors. For a variety of reasons, however, there has not been a good record of applying relevant research results related to flightcrew performance and the flightcrew-automation interface. Some research results go unused simply because those who can apply them are unaware of the research. Other research goes unused because the results are incomplete, or they are in an unusable form. Vital pieces of information may be missing, or the research may not have been carried far enough to use it in a commercial application. Finally, industry is sometimes reluctant to incorporate technology and other research results that were not developed in-house. These difficulties in applying human factors research results have contributed to the inadequacies of the data, tools, and guidance available to designers, operators, and regulators, particularly evaluation tools and methods for evaluating human performance.

The HF Team found that many communication breakdowns are occurring in the research project definition and results transfer process. Not only are potential users sometimes unaware of relevant research results, but also researchers are not always aware of the needs and constraints of airplane design, operation, and certification. The HF Team believes that researchers and research sponsors need to become more actively involved in seeking out practical research needs and constraints, and in supporting the transfer of results. Regulators/designers/operators should ensure that their needs and constraints are communicated and that processes are established to disseminate and use applicable research results.

The HF Team also found that measures of success for research projects are often not oriented toward technology transfer. Success appears to be more often determined by publishing a paper, holding a symposium, or obtaining continued funding, rather than practical application.

Another concern of the HF Team is that some of the research being done in flight deck human factors consists of separate projects that are not well coordinated. Although there may be organizational and ad hoc ties between researchers, no single organization 
identifies and tracks high-level research needs on a national or international level. For example, the issue of mode awareness has been recognized for several years. Yet, no organization has identified the high-level research (and any other) needs that would provide the complete results, guidelines, and data necessary to resolve this problem from a design, regulatory, training, and operations perspective. As a consequence, there are examples, such as the many individually very good and relevant projects addressing mode awareness, that have not been planned and coordinated in such a way to ensure that the issue will be fully addressed.

\section{Recommendations}

\section{Recommendation Comm/Coord-1}

The FAA should identify existing air traffic procedures that are incompatible with highly automated airplanes. These incompatible procedures should be discontinued or modified as soon as feasible.

\section{Discussion of Recommendation Comm/Coord-1:}

This recommendation is intended to provide a short term solution to the incompatibility problems that currently exist between highly automated airplanes and the air traffic system. In cooperation with system users, the FAA should identify and resolve any particularly difficult or troublesome procedures. Example candidates for evaluation include complex departures or arrivals into major hubs, excessive descent gradients, VOR radial intercepts or crossing constraints that are not well suited to FMS operations.

With ATA support, the FAA should consider requesting the ATA/FAA Flight Management System Task Force to identify incompatible procedures as candidates for discontinuation or modification.

\section{Recommendation Comm/Coord-2}

The FAA should task an existing advisory group or, if necessary, establish a new forum to ensure coordination between the design of air traffic procedures and the design and operation of highly automated airplanes.

\section{Discussion of Recommendation Comm/Coord-2:}

This recommendation is intended to provide a long term solution to current and future incompatibilities between highly automated airplanes and the air traffic service

environment. Representation in this forum should include airplane operators, airplane and 
avionics manufacturers, pilot groups, air traffic service providers and regulatory authorities, as well as other users of the air traffic system and suppliers of airplane and air traffic systems equipment as necessary. This forum should coordinate closely with other national and international bodies dealing with airspace and air traffic procedure issues to ensure that overlap, duplication of effort, and conflicting solutions are minimized.

International participation is appropriate to address the incompatibility issue on a global basis.

This forum should be used to ensure that, within the constraints imposed by other airspace users, air traffic procedures make optimum use of advanced airplane automation in terms of safety as well as economic efficiency. Airplane automation should be designed to allow the flightcrew to easily comply with air traffic procedures and vice versa.

Implementation of the airplane automation should be such that different airplanes performing the same procedure do so in a similar manner for both safety and system efficiency reasons. For example, when flying from one altitude constraint to the next in VNAV, all airplanes should be capable of satisfying critical constraints in a consistent manner (e.g., by using the same type of flight path between altitude constraints). This would aid both flightcrews and air traffic personnel relative to their expectations of the airplane's flight path.

Coordination efforts similar to what the HF Team is recommending on a system-wide basis have been undertaken on a limited scale in response to the challenges of certain new capabilities (e.g., data link, free flight). The HF Team is concerned that these coordination efforts are not institutionalized and are not occurring on an international basis such that criteria, equipment, and procedure changes are systematically evaluated for potential incompatibilities. Also, efforts that end when the new capabilities are introduced cannot resolve problems that may arise on a continuing basis as the CNS systems evolve.

\section{Recommendation Comm/Coord-3}

The FAA should lead an industry-wide effort to share safety information obtained from in-service data and from difficulties encountered in training. This effort should be capable of assisting in the identification and resolution of problems attributed to flightcrew error.

\section{Discussion of Recommendation Comm/Coord-3:}

The HF Team recognizes that many issues must be resolved for industry-wide data sharing to occur, especially on an international basis. The HF Team notes both the significant progress that has been achieved thus far on this very important safety initiative and the commitment from the aviation community to implement it. The HF Team strongly supports this effort. We recommend that these efforts be accelerated. As noted in this report, problems currently attributed to flightcrew or other human errors are frequently 
associated with underlying deficiencies in design, flightcrew qualification and training, operational procedures, or other sources, and should not be focused exclusively on flightcrew performance.

Apart from the legal issues that must be surmounted, the HF Team notes that the success of this initiative relies on the quality of information produced, the quality of the analysis performed, and the communication of this information to those who can take meaningful action.

For the future, improved data collection techniques should be considered, such as internal and external video cameras, to record information that is currently unavailable from flight data, cockpit voice, and quick access recorders. Such information could include a more complete picture of mode annunciations, display states, and other visual feedback available to the flightcrew, as well as external factors not easily captured on an electronic data bus.

Because it will probably be some time before a program is fully implemented, the HF Team recommends that the FAA consider expanding the use of the Aviation Safety/Accident Prevention (ASAP) system to fulfill some of the these objectives. The FAA's Rotorcraft Directorate is currently using this system to track significant in-service problems. ASAP information can be made available to authorized users with a computer and a modem, including manufacturers and operators.

\section{Recommendation Comm/Coord-4}

The FAA should require operators to have an appropriate process, with demonstrated effectiveness, for informing flightcrews about relevant accidents, incidents, in-service problems, and problems encountered in training that could affect flight safety.

\section{Discussion of Recommendation Comm/Coord-4:}

This recommendation addresses the HF Team's concern that information about known safety problems may not be brought to the attention of flightcrews, or that such safety information may not be sufficiently emphasized. Operators should have an effective process, coordinated with the FAA, for accomplishing this task. In order to assure effectiveness, operators should demonstrate that relevant safety information is effectively conveyed, understood, and put to use by flightcrews. Implementation of this process could lead to changes in training, operational procedures, standard operating practices, policies, etc. following an accident, incident, in-service-problem, or problem encountered in training. When changes are made, the reasons for the changes should be explained to flightcrews.

Operators may incorporate this process into their existing programs or they may use or develop new means of communicating this information to flightcrews. This 
recommendation is primarily directed at Part 121 and/or Part 135 operators; however, this process may also be useful for Part 91 operations.

\section{Recommendation Comm/Coord-5}

The FAA should encourage the redesign and modernization of the information provided to the flightcrew in notices to airmen (NOTAMs), charts, approach plates, instrument procedures, meteorological data, etc. The information should be prioritized and highlighted in terms of urgency and importance, and presented in a clear, well-organized, easy-to-understand format suitable for use with current and future airplanes.

Discussion of Recommendation Comm/Coord-5:

Information to flightcrews should be presented in an easy-to-read format with important information highlighted. For example, information on ground navigation equipment outages that could affect FMS navigation or cause map shifts should be suitably emphasized.

Because the problem this recommendation addresses is international in scope, an internationally harmonized solution should be obtained. This effort should be addressed by a working group consisting of the developers, distributors, and users of this information.

\section{Recommendation Comm/Coord-6}

The FAA should improve and increase interaction between the Flight Standards and Aircraft Certification Services.

\section{Discussion of Recommendation Comm/Coord-6:}

Increased involvement of the AEG in type certification and supplemental type certification projects is needed to ensure that operational suitability is adequately addressed. This is particularly true for projects involving ACOs that are not co-located with an AEG, or for avionics projects approved on Part 23 airplanes that are later extended to Part 25 air carrier airplanes. Human factors issues may be more readily identified and resolved if the AEG is involved to provide a better understanding of the operational environment in which the airplane will be operated.

Increased participation by ACO specialists is needed in FOEBs and FSBs to improve the quality and efficiency of the process used to develop Master Minimum Equipment Lists and flightcrew qualification criteria. 
The HF Team realizes that organizational changes currently being planned or implemented within the FAA may affect the implementation of this recommendation. Without presupposing the effects of potential organizational changes, one way to implement this recommendation would be to form a group of appropriate Flight Standards and Aircraft Certification personnel to develop guidelines for improving interaction between the two services. This group should have representation that is balanced and cognizant of the difficulties in existing communication and coordination between the Services. All Flight Standards and Certification Service personnel should be informed of the group's goals and progress, and be given an opportunity to provide input.

\section{Recommendation Comm/Coord-7}

The FAA and industry should improve the coordination and distribution of tasks undertaken by federal advisory committees and industry technical committees to reduce overlap and avoid duplication of effort.

\section{Discussion of Recommendation Comm/Coord-7:}

The FAA and industry should identify current working groups and technical committees, their membership, and the tasks they have been assigned. A team approach is needed to update charters for these groups such that overlap and duplication of effort are minimized. Groups working on similar or related tasks should be aware of each other, and formal lines of communication and coordination should be established between them.

\section{Recommendation Comm/Coord-8}

The FAA should improve communication about research programs, research results, and advances in technology to appropriate FAA personnel.

\section{Discussion of Recommendation Comm/Coord-8:}

FAA personnel should be made aware of research programs, research results, and technology advances that are relevant to their area(s) of expertise. There are many ways this recommendation could be accomplished, but an important consideration is that it be institutionalized. Means of accomplishing this recommendation could include any or all of the following: FAA or outside briefings, training and education programs, newsletters, membership in technical or professional organizations, subscriptions to technical or industry journals, and partnerships with NASA, academia, and industry.

The implementation of this recommendation should be integrated with the implementation of Recommendation Knowledge-13. 


\section{Recommendation Comm/Coord-9}

The FAA should hold research funding sponsors and researchers accountable for supporting the transfer of research results.

Discussion of Recommendation Comm/Coord-9:

In providing funding for research, the FAA should require contractual obligations for qualified reviews and for supporting the transfer of research results to specified target customers, which may not be the same as the funding organization. Technology transfer requires additional efforts and resources beyond the research itself (although it should not be considered to be a completely independent activity). Resources for transferring the results should be considered as high a priority as conducting the research, and the program should be considered incomplete until the results have been reviewed for potential transfer. The FAA should encourage other funding organizations to adopt similar criteria.

\section{Recommendation Comm/Coord-10}

The FAA should assure strategic leadership and support establishment of a coordinated research portfolio in aviation human factors on the national and international levels.

\section{Discussion of Recommendation Comm/Coord-10:}

Better coordination should be established between the FAA (including each of the major Services), NASA, the Department of Defense, the aviation industry, academia, and possibly the National Science Foundation and National Research Council to provide strategic leadership in aviation human factors on the national level. This activity should coordinate the appropriate international human factors activities (government, industry, academia) to provide strategic leadership on the international level. Representation in this activity should include a mix of skills and backgrounds, including strong representation from manufacturers and operators.

This effort should be coordinated with the National Plan for Civil Aviation Human Factors to assure a well-planned research portfolio. Responsible organizations must have sufficient authority, both in terms of funding and program planning, to ensure that a coordinated national research program can be effectively managed. There must be accountability for developing and implementing a relevant and effective research portfolio as well as facilitating the successful transfer of results. The activity should include a means to stay abreast of relevant research efforts conducted in other industries or areas and ensure that results are considered for their applicability to aviation. 
The research portfolio should reflect a balance of appropriate factors, such as long term and short term needs, and operational, engineering, and scientific goals. The costs and benefits of each project should be assessed in terms of how the project fits into the overall portfolio and the expected value of the potential results. A well-coordinated research portfolio would avoid unnecessary duplication of effort and to ensure that a critical mass of research activities is constantly being undertaken to resolve particular problems. 


\section{Processes for Design, Regulatory, and Training Activities}

This section addresses concerns the HF Team has regarding the processes used for design, regulatory, and training activities for the airplane types in our charter.

\section{Design}

"Human-centered automation: Automation designed to work cooperatively with human operators in the pursuit of stated objectives.

- Dr. Charles Billings ${ }^{13}$

"Recognize the pilot's world and design the airplane to fit it. A more human-centered design is needed.”

- A major U.S. airline, identifying one of their top concerns to the HF Team

\section{Improvements necessary in the application of human-centered design}

The development and introduction of advanced automated systems have increased the efficiency, precision, and safety of airplane operations. For the present and projected airplane environment, current glass cockpit airplanes are easier to operate, in most respects, than their immediate predecessors (e.g., DC-9, B727, etc.). However, while these highly automated aircraft are generally easier to operate in normal circumstances, or in non-normal circumstances that are provided for in failure scenarios addressed by the design (e.g., automatic electrical system reconfiguration following an electrical generator failure), operations can become very confusing if the expected response does not occur, or if a novel malfunction or unusual combination of malfunctions occurs. The flightcrew must be able to understand the automation's status and behavior, especially during unusual or demanding situations.

Current automated cockpit systems have a high level of both autonomy and authority and the systems have become more complex and numerous. However, the objectives of their inner functioning may not always be obvious to the flightcrew. The HF Team heard evidence of this during our meetings with the operators (and pilot groups). As discussed in the section on "Flightcrew Management and Direction of Automation," we found that pilots are still asking questions such as "What's it doing now?" "Why did it do that?" and "What will it do next?" in reference to the actions of cockpit automated systems. We

\footnotetext{
${ }^{13}$ Billings, Charles E., Human-Centered Aircraft Automation: A Concept and Guidelines. NASA Technical Memorandum 103885, August 1991.
} 
heard directly from one operator about the concern that "some recent airplane designs are not pilot-centered." Another operator stated that one of their most important concerns is having the airplane do something unexpected (e.g., not knowing what mode the airplane is in, uncommanded mode changes, or unannunciated mode changes). The ASRS contains numerous examples of breakdowns in interactions between the flightcrew and the automation. (Refer to Appendix F for examples of pilot reports extracted from the ASRS.) While flightcrew qualification and training can address some of these issues, it is noteworthy that these questions are also being asked by flightcrews who may have years of experience and thousands of hours of experience in a particular airplane type. In such instances, it is clear that training is not the only answer.

Each airplane manufacturer has a different philosophy regarding the implementation and use of automation. However, there is general agreement that the flightcrew is and will remain ultimately responsible for the safety of the airplane they are operating.

The way pilots operate airplanes has changed as the amount of automation and the automation's capabilities have increased. Automation has both provided alternate ways of accomplishing pilot tasks performed on previous generations of airplanes and created new tasks. The pilot has become, in some circumstances, a supervisor or manager of the automation. The increased use of and flightcrew reliance on flight deck automation makes it essential that the automation act predictably with actions that are well understood by the flightcrew.

The HF Team believes that flight deck automation must provide the flightcrew with appropriate information about its intended course of action. The system must support the flightcrew's ability to maintain a high level of awareness about the automation status, behavior, intention, and limitations in order to allow flightcrews to reliably and efficiently coordinate their activities with the system.

Moreover, the automation must be designed to function in a manner that directly supports flightcrews performing their tasks. If these human-centered design objectives are not met, the flightcrew's ability to properly control or supervise system operation is limited, leading to confusion, automation surprises, and unintended airplane responses.

During our visits to the airplane manufacturers involved in this study, we saw evidence that they utilize human-centered design principles to varying degrees when developing a flight deck design. But our Team also found evidence that points to areas where the application of these principles by each manufacturer could be improved. The HF Team examined how the manufacturers address human factors issues in the design process, and noted the following:

- Automation design principles are often not defined, documented, or distributed to appropriate design, test, or training personnel.

- Some flightcrew cognitive tasks are not comprehensively identified or considered in the design. 
- Flightcrew information and feedback requirements are not always clearly identified or given high priority in making design tradeoffs.

- During the design process, flightcrew task allocation is not clearly identified either between flightcrew members or between the automation and the flightcrew. This can result in imbalances between tasks allocated to the pilotnot-flying versus pilot-flying.

- Designers sometimes make flight deck and display design decisions based on subjective assessments in balancing flight test pilot input, chief pilot or project pilot input, operator input, and economic input instead of being data- or service-history driven.

The HF Team supports the concept that the pilot's interface with the system, including task needs, decision needs, feedback requirements, and responsibilities, must be primary considerations for defining the system's functions and logic, as opposed to the system concept coming first and the user interface coming later, after the system's functionality is fully defined. ${ }^{14}$ The HF Team's assessment of recent designs found numerous examples where application of human-centered design principles and processes could be better applied to improve the design process and final product. The HF Team believes that without more effort in this area we will continue to see pilot-automation communication breakdowns resulting in potential future automation related incidents and accidents.

\section{Importance of recognizing human factors as a core discipline}

Although each manufacturer utilizes human factors specialists to varying degrees, they are typically brought into the design effort in limited roles or late in the process, after the operational and functional requirements have been defined. When joining the design process late, the ability of the human factors specialist to influence the final design and facilitate incorporation of human-centered design principles is severely compromised. Human factors should be considered on par with other disciplines involved in the design process.

Further discussion of related issues can be found in the section on "Knowledge and Skills of Designers, Pilots, Operators, Regulators, and Researchers."

\section{Regulatory Processes}

"The next step is obvious: we must include Human Factors requirements into the certification processes of people, procedures, and technology, so that Human Factors issues are considered at the time when we are defining the blueprint of our system, before

\footnotetext{
${ }^{14}$ Riley, Victor, What Avionics Engineers Should Know about Pilots and Automation. Honeywell Technology Center.
} 
it is operational and not after. This is, in my view, a cost-effective approach to anticipate human error rather than regretting its consequences."

Mr. Jack Howell, Director, Air Navigation Bureau, ICAO, addressing the Opening Session of the Third Global Flight Safety and Human Factors Symposium, Auckland, New Zealand

\section{Improvements necessary in the type certification process}

Whereas incorporating human-centered design principles is important, so is the regulatory evaluation of the resulting systems to assure consideration of those principles. Current regulations and associated guidance material do not provide criteria that encourage or require manufacturers to develop and follow a flight deck design process that comprehensively addresses human performance considerations. In addition, except for flightcrew workload, the existing regulations and advisory material do not provide regulatory authorities with the criteria and methods they need to conduct an evaluation of human performance issues associated with the design (refer to the section on "Criteria, Regulatory Standards, Methods, and Tools for Design and Certification"). Often, evaluation of the overall flight deck design by regulatory officials is primarily conducted near the end of the design cycle, during flight testing. This occurs late enough in the design process that it is often difficult to make desirable design changes that have been identified during the evaluation.

Flight test evaluation is able to address many human performance concerns, but cannot address them all. In some cases where it is considered too expensive to change the design, a procedure is developed to address the concern. An effort must be made to minimize this method of fixing vulnerabilities in the design. The concern here is that the "fix" may mask the real problem, and if this operational procedure should be revised or eliminated sometime in the future, the original design problem may become a hazard. Clear and concise regulatory criteria and methods used during the flight deck certification process would help in defining the boundary between unsatisfactory and unsafe features, and thereby reduce this concern.

Another concern is that the personnel doing the evaluations may not have up-to-date information necessary to make the evaluation. Refer to the section on "Knowledge and Skills of Designers, Pilots, Operators, Regulators, and Researchers.”

In summary, the HF Team considers that the current type certification (TC) process does not adequately incorporate human factors considerations in the design and evaluation of flight decks.

\section{Improvements necessary in the supplemental type certification process}

Any individual or company can apply to modify an existing type-certified airplane through the supplemental type certificate (STC) process. These individuals or companies are not 
necessarily cognizant of design decisions made by the airplane manufacturer regarding the flight deck design philosophy, nor of the operating assumptions used. Just as a particular STC applicant may be unfamiliar with these important considerations, so may regulatory personnel located in an ACO other than the one responsible for issuing the original type certificate (i.e., ACOs located outside the home region of the airplane manufacturer). Therefore, the potential exists for an applicant to propose and receive approval for a flight deck modification that is not in accordance with the manufacturer's flight deck design philosophy and operating assumptions. The HF Team is concerned about the potential safety impact of such modifications.

One major airplane manufacturer suggested that they should be included in the review process for proposed modifications to its flight deck design. This suggestion addresses the concern that a good (i.e., safe) design may be corrupted via an STC that does not fully consider the original design assumptions or characteristics. While the HF Team does not believe the airplane manufacturer should necessarily be included in reviewing proposed flight deck modifications, (i.e., have a "vote" in the approval process), this deficiency in the method by which certain STC applications are currently reviewed and approved by the FAA has been noted, and the HF Team concurs with this assessment.

The current review process within the FAA requires that all ACOs notify the TAD of proposed STC modifications to transport category airplanes. The TAD Standardization Branch (ANM-113) is tasked with conducting reviews of the modifications and following up on those that appear to be major or otherwise significant changes to the original type design. The HF Team is concerned that this process is a weak defense against possible design incompatibilities. The large quantity of proposed certification projects does not always allow adequate review by the Standardization Branch staff. In addition, the description of the project sent to the TAD takes place in summary form that sometimes fails to fully identify the proposed modification, much less describe it in detail. Also, notification of the modification sometimes occurs after the approval process is well underway, or sometimes even after it has been completed.

Developing and documenting the intended functionality, philosophy, and design decisions of the original flight deck design through a formalized process would help ACO personnel during the review and approval process for proposed STC modifications by giving them a basis for comparison. A similar process applied to STC modifications would also minimize potential additional design incompatibilities with the original design when subsequent modifications to the STC are proposed.

\section{$\underline{\text { Inconsistent regulatory results }}$}

A lack of objective, measurable regulatory standards, processes, and tools for evaluating human performance can contribute to inconsistent regulatory results, because certification personnel then use subjective judgment that can vary between individuals. This can lead to different regulatory decisions and inconsistent means of compliance being imposed on operators and manufacturers. This issue can arise on different airplane types for the same 
manufacturer, and for different manufacturers. It can even occur on the same airplane type when modifications are certified at different ACOs. In the absence of clear, objective, quantifiable standards, individual's opinions become the standard against which the design is measured.

\section{Inflexibility in adapting to new technologies and inappropriate use of precedence in certification discourages use of updated knowledge}

The Team heard from operators and manufacturers that the FAA and other regulatory authorities should allow more flexibility in adapting to "real world problems and new technology. They claim that current regulatory standards can inhibit the introduction of new technology. One example of the type of problems that can occur is given by a recent attempt by one operator to gain approval for the use of GPS for navigation. The proposed regulatory criteria to integrate the GPS sensor into the existing navigation system resulted in standards the applicant believed were unjustified, inappropriate, and too costly. The regulatory basis proposed by the cognizant ACO was based on an inflexible FAA policy that was intended to ensure a minimum level of safety. The applicant ultimately canceled the project. Yet there was general agreement that using GPS for area navigation and instrument precision approaches (the applicant's goal) would have had many benefits, including increased safety. The HF Team believes that improving the certification process and revising existing criteria and methods (or developing new criteria and methods) could assist the applicant and the regulatory community to achieve the goal of incorporating desirable new technology, while maintaining or increasing aviation safety.

In addition to the inflexibility of the current rules to adapt quickly to new technologies, applicants often successfully use the argument of "we certified it that way before, why can't we do it that way now?" (so called "grandfather rights"). While in some cases this can be a valid argument, in many other cases it is not. Using precedence in this way can inhibit the use of updated knowledge of potential safety problems, regardless of what certification criteria were applied in the past. Unfortunately, depending on the proposed modification, this use of precedence can have potential adverse safety implications. The HF Team believes that a clear and consistent policy is needed regarding the use of precedence in certification.

\section{Training}

"One of the myths about the impact of automation on human performance is -- as investment in automation increases, less investment is needed in human expertise. In fact, many sources have shown how increased automation creates new knowledge and skill requirements."

- Dr. David Woods 
In our investigations, we heard from operators that the subtle nature and complexity of automated flight decks result in the flightcrews needing additional knowledge about how the different automated subsystems and modes function. Industry investigations have shown that the complexities of the automated flight decks make it easy for pilots to develop oversimplified or erroneous mental models of system operation, particularly mode and transition logic. Training departments tasked with developing and teaching flightcrews how to manage the automated systems in differing flight situations confirm this finding. Many sources offered incidents where pilots were having trouble getting a particular mode or level of automation to work successfully, and where they persisted too long in trying to make the automation carry out their intentions instead of switching to another means to accomplish their flight path management goals. We heard how the new knowledge and skill demands are most needed in unusual situations where different or extraordinary factors push the chain of events beyond the routine. It is just those circumstances that are most vulnerable to a breakdown in reliable human-automation performance through a progression of misassessments and miscommunications. Contrary to the content of some qualification programs, the HF Team believes it is important for flightcrews to be prepared by their training (as opposed to "picking it up on the line"), so that they will be prepared to successfully cope with probable, but unusual situations.

For training managers and departments, the result is the need to address training demands that may need to be fit into a small and shrinking training footprint. Various strategies have been developed to cope with this situation. For example, one strategy is to focus transition training on a basic set of modes and leave alternative methods to be mastered during line operations. This can lead to training those parts of managing automated systems that are the easiest to learn, while deferring the more complicated functions, and functions where vulnerability is higher, for individuals to learn later on their own. This method is suitable only if the airplane can be safely operated with the set of skills mastered and:

- If the basic skills provide a coherent base that permits learning the more difficult skills, and

- If there is an environment that assures mastery of necessary advanced skills before they are operationally needed (e.g., oceanic environment, autoland, etc.).

Another strategy is to teach rote step-by-step procedures backed up by manuals or quick reference guides. Training organizations typically are justifiably uncomfortable with this method and therefore try to go beyond rote training as much as time and resource limits allow.

Regardless of methods, pilots must have the opportunities to practice what they have learned in realistic operational settings through Line Operational Simulations (LOS) and LOFT scenarios, or Initial Operational Experience (IOE). The HF Team believes that it is important for the industry to get better utilization from limited training time available and 
the limited testing or assessments now conducted (e.g., checks, Line Operational Evaluation, etc.).

As training footprints shrink, or as more knowledge or skill items must be addressed, it becomes increasingly important to assure that critical knowledge and skills are mastered.

The industry wants and should have increased freedom to focus limited training resources on areas that yield high training effectiveness (as in certain aspects of the Advanced Qualification Programs (AQP) under development). However, this flexibility should not be used to reduce training and practice, but instead it should be used to better address high priority training needs in areas where service experience indicates vulnerabilities.

Economic pressure to take AQP benefits in productivity improvements (reaching the same goal faster) rather than in safety or quality improvements (more effective training) should be resisted, as long as high priority training is going unaddressed (e.g., automation management). Trying to squeeze more yield from a shrinking investment in human expertise will not help prevent the kinds of incidents and accidents that are currently being labeled as human error.

\section{Recommendations}

\section{Recommendation Processes-1}

The FAA should task an aviation industry working group to produce a set of guiding principles for designers to use as a recommended practice in designing and integrating human-centered flight deck automation.

\section{Discussion of Recommendation Processes-1:}

The objectives of these guiding principles would be to provide a framework for design engineers to incorporate human-centered design principles into future flight deck designs. The effort should include representation from the airplane and equipment manufacturers, operators, flightcrews, and human factors specialists from industry and the research community.

The HF Team suggests that such an effort consider the following criteria and principles:

- Flightcrew-centered design should be explicitly addressed.

- Design principles should be documented and available to designers.

- Designers should be knowledgeable about applicable human factors principles and guidelines.

- Human factors expertise should be represented on the design team. 
- Pilot opinion as a basis for design decisions is necessary, but is not sufficient by itself. Structured scenarios using suitable subject pilots should be used to investigate flightcrew performance issues.

- Human factors should be considered early in the design process and should be part of the entire design process.

- Flightcrew functions, tasks (including physical and cognitive tasks) and associated information requirements should be explicitly identified as part of the design process.

- Designs should accommodate the range of expected pilot behaviors.

- Salient feedback on automation status and behavior is necessary.

- Design, training, and operations should be tightly coupled.

- Absolute evaluation criteria should be developed and used in addition to relative criteria.

- A formal systematic process should be developed for evaluating the flightcrew/automation interface.

- Manufacturer/operator/user communication is necessary (not just at the management level).

- International/cultural effects should be considered in the design.

\section{Recommendation Processes-2}

The FAA should establish regulatory and associated advisory material to require the use of a flight deck certification review process that addresses human performance considerations.

\section{Discussion of Recommendation Processes-2:}

An FAA/JAA working group comprising FAA/JAA certification engineers, flight test and operational (i.e., from AEG) pilots, human factors specialists (from FAA, JAA, industry, and any other interested parties), and other industry personnel should be formed to implement this recommendation.

New regulations should be developed to require reviews throughout the development and certification period. The regulatory and advisory material, based on the following principles, should be applicable to new and amended type certificates (consistent with the requirements of Part 21 of the FAR) as well as supplemental type certificates:

(a) The flight deck certification process should validate the overall integrated design of the flight deck including:

- Operational acceptability of flight deck displays, 
- Ease of use, and understandability of system operating modes and logic,

- Control layout and labeling,

- System state and mode feedback,

- Compatibility between various systems in the flight deck,

- Flight deck layout ergonomics,

- Potential for error and/or susceptibility for inducing error, and

- Consistent use of:

- color

- nomenclature

- method of operation

- control laws

- data sources
- symbology

- controls

- alerts, voice syntax

- processing algorithms

- data reference

In addition, the process should verify adherence to human-centered design guiding principles that may be adopted or developed by the airplane manufacturer.

(b) Consideration of human factors and human performance in the certification process should begin at the early stages of a system design, be iterative, continue through all stages of design, and involve HF expertise. Periodic certification and operational reviews should be conducted to detail how human performance considerations are being taken into account in the design.

Figure 6 presents a graphical representation of an example design process showing a typical point where some aspects of human performance/human centered design principles should be included in the design decision process.

(c) Applicants should demonstrate that a design is acceptable for use by flightcrews flying in the expected operational environment. Criteria for acceptability of these demonstrations should consider actual pilot performance using similar equipment (when such information is available from service history). This should consider pilot behavior from pertinent operating environments, cultural backgrounds, experience levels, and flightcrew qualification profiles. 


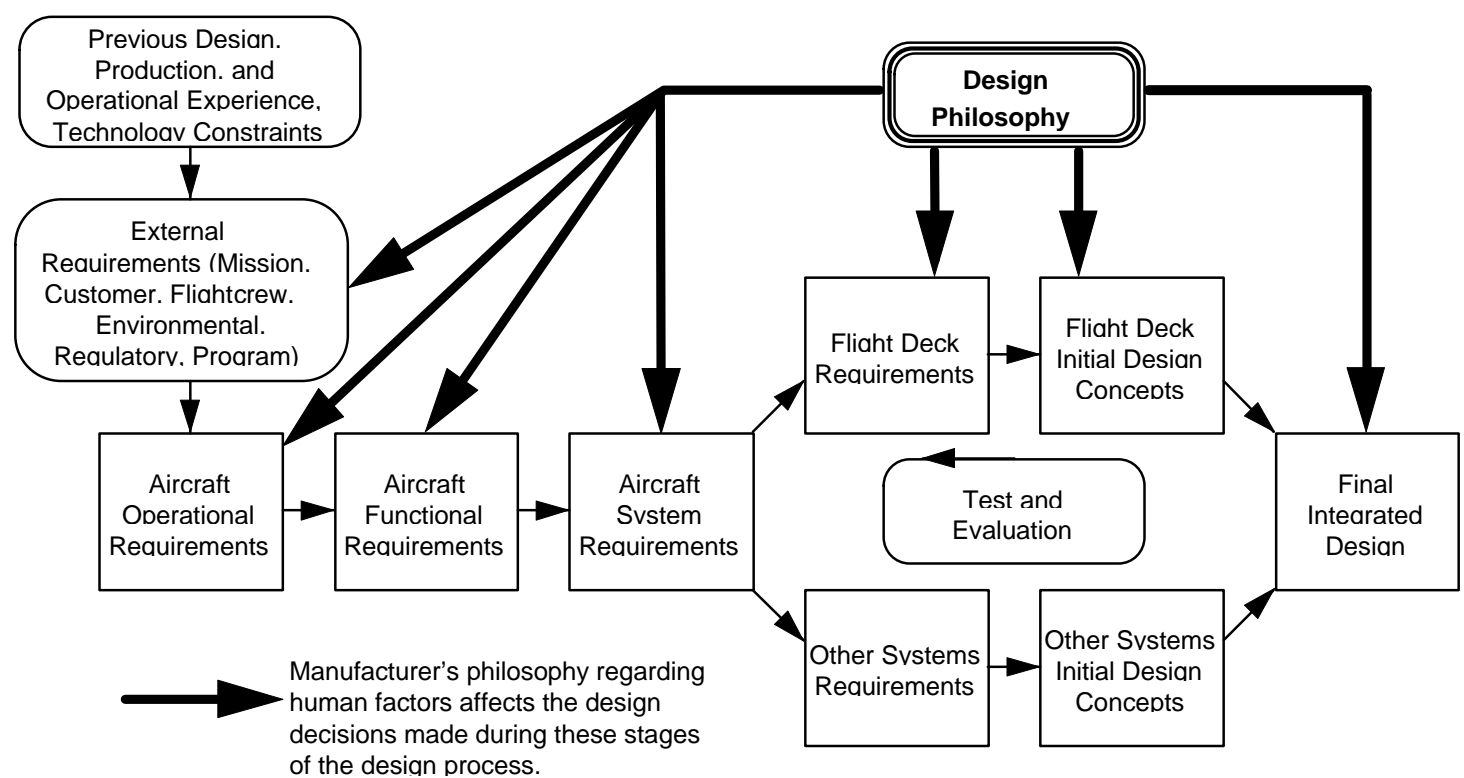

Figure 6

Example Design Process Incorporating Human Factors Design Principles ${ }^{15}$

(d) Assumptions made for the certification of previous airplane types should be reviewed, and not necessarily assumed as precedents for future developments. New designs need to be reviewed in light of:

- Flightcrew performance in the air traffic system environment(s) in which the aircraft is expected to be operated,

- Expected pilots' behavior patterns, and

- The addition of new equipment (via new TC, amended TC, or STC) for which there is no service history.

(e) The flight deck design must support the flightcrew in their primary task of flying the airplane. Identifying flightcrew tasks and information requirements is important so that designers and evaluators can ensure that design objectives are met. To do this, the following steps are important:

- Flightcrew cognitive and physical tasks should be identified to some appropriate level of detail.

- Flightcrew information and coordination requirements should be identified.

\footnotetext{
${ }^{15}$ Palmer, Michael, T., William H. Rogers, Hayes N. Press, Kara A. Latorella, and Terence S. Abbott. A Crew-Centered Flight Deck Design Philosophy for High-Speed Civil Transport (HSCT) Aircraft. NASA Technical Memorandum 109171. January, 1995.
} 
- Flightcrew task allocation should be identified between flightcrew members and between the automation and the flightcrew.

(f) Applicable aspects of the flight deck certification process should be performed for STC applications that introduce changes to the flight deck. Regulatory authorities should evaluate the proposed modifications to ensure that the airplane manufacturer's original design philosophy and operating assumptions, considered during the original type certification process, are not adversely affected. The TAD Standardization Branch, ANM113, should be responsible for ensuring that the ACO that issued the airplane's TC is appropriately included in the review of the proposed modification. In the case of an airplane manufactured outside of the United States (airplanes certified under $§ 21.29$ ), ANM-113 should ensure that the cognizant foreign airworthiness authority is appropriately involved in the review. In addition, the appropriate AEG should be included in the review to ensure that operational considerations are adequately addressed.

Criteria should be developed for conducting STC reviews in a standardized manner. In addition to the criteria discussed in Paragraphs (a) - (e) above, the review should consider the following issues depending on the modification:

- Operator workload;

- Access to controls;

- Flightcrew ability to view and understand displays;

- Acceptability of feedback and mode awareness; and

- Error potential.

This process (along with other existing and new HF-related criteria) would provide HF tools that could help in minimizing the subjective nature of the current certification process and reducing future potential design feature vulnerability that may not otherwise have been identified.

If new or updated regulatory criteria are adopted, there should be a parallel development of associated advisory material. The regulatory and advisory material must be written in a way that can be practically applied by the applicant, and are clear about what the applicant must do, when the applicant is finished, and what criteria will be used to judge acceptance. The material should address acceptable processes that can be used, including methodology, rather than specific design requirements. The proper roles, safety objectives, and the relationships between associated airworthiness and operating rules must also be respected. In addition, we recommend that the standards and criteria be harmonized internationally. 


\section{Recommendation Processes-3}

The FAA and the aviation industry should investigate the use of innovative training tools and methods to expand pertinent safety related knowledge of flightcrews on a continuing basis. The FAA and the aviation industry should explore incentives to encourage continued training and education beyond the minimum required by the current regulations.

\section{Discussion of Recommendation Processes-3:}

The FAA, operators, pilots' associations, training organizations, airplane manufacturers, and other interested parties should implement this recommendation through existing working groups addressing training issues (e.g., the ATA training committee).

The HF Team suggests consideration of the following principles for improving the training process:

(1) Invest in more line-oriented practice and address areas of known vulnerabilities.

- Create a larger set of line-oriented scenarios to practice.

- Update these scenarios regularly to reflect the latest information about vulnerabilities from incident reporting systems or other sources.

- Expand scenarios to focus more on unique error-vulnerable situations.

(2) Invest in more coaching and less pass/fail testing.

- Improve the debriefing of flightcrew performance after simulator sessions, IOE, proficiency checks, etc. (e.g., standardization of instructor debriefs, video replays).

- Focus more on practicing how to manage the different automated systems in different circumstances, especially the judgments that have to be made on transitioning between different levels of automation (e.g., when to turn it off or on, or to change to a different level or mode).

- Encourage initial/recurrent assessments or checks to be more "learning oriented." Emphasis should be focused so that learning becomes the primary objective rather than passing or failing. In addition to using time better, such a system might incorporate progressive assessment of individual elements/maneuvers or event sets. Assessment may also provide for levels of individual performance based on a graduated scale, rather than an "all or nothing" grading system that may diminish opportunities for learning. Although qualification processes must also recognize and provide for those instances when there is unsatisfactory performance, different grading scales might be possible (e.g., a limited number of repetitions permitted to achieve acceptable performance).

(3) Support exploration 
- Use automation surprises that occur on the line as subsequent training opportunities to learn more about the automation and how to manage it.

- Support follow-up of automation surprises in a simulator environment in LOFT scenarios or line operational evaluations.

- Provide more opportunities to learn and practice, especially how to handle surprising situations.

- Identify and correct oversimplifications in pilots' mental models of system functions.

- Promote understanding rather than using rote training.

(4) Create an environment that rewards and supports continual learning.

- Treat mistakes and errors as opportunities for learning.

- Allow sufficient time for questions and thorough understanding.

- Challenge flightcrew members to further develop their skills through the use of appropriate incentives.

Initial and recurrent training should provide a clear understanding of operationally relevant automation principles and ensure user proficiency for the cockpit automated systems, including how these systems are used in conjunction with other systems (e.g., autopilot use during engine failure). (Refer to the section on "Knowledge and Skills of Designers, Pilots, Operators, Regulators, and Researchers" for discussion of initial and recurrent training.) The HF Team recognizes that initial, recurrent, differences, and transition training programs are limited in the amount of knowledge that can be taught because of the short periods of time available for such training. As automatic systems become increasingly complex, the range of features available for use by the flightcrew grows. Even if every system feature is covered and practiced during initial, recurrent, differences, or transition training, it is not certain that the pilot will necessarily retain all of the information. Continuous learning is one way to help ensure that pilots have the knowledge they will need in order to effectively manage and use the automation in a wide range of situations.

There are other areas related to automation where continuing education would also be beneficial. These areas include, for example, meteorology, principles of modern navigation system functions, aeromedical knowledge of fatigue and error vulnerabilities, advanced functions of specific systems like ground sensing and anti-skid in adverse conditions, advanced FMS applications, and training aids for CFIT and windshear. 


\section{Criteria, Regulatory Standards, Methods, and Tools for Design and Certification}

This section addresses the HF Team's concerns regarding inadequate criteria, regulatory standards, methods, and tools for design and certification of highly automated airplanes.

\section{Inadequate Criteria, Regulatory Standards, Methods, and Tools for Evaluating Flightcrew Performance}

In examining flightcrew errors, the HF Team looked at design, training and flightcrew qualification, operations, and regulatory processes, to understand factors contributing to the errors. Often, flightcrew errors can be easily identified in hindsight, and it can be postulated that many of the errors are predictable and are induced by one or more factors related to design, training, procedures, policies, or the operating environment. The more difficult task is to anticipate these errors and take preventive corrective action prior to occurrence of a potentially hazardous situation. The HF Team believes it is necessary to improve the ability of airplane manufacturers, airworthiness authorities, and operators to detect and eliminate design characteristics (or features) that create predictable errors.

Current regulatory criteria do not comprehensively address the evaluation of flight deck designs for their contribution to flightcrew error or to human performance problems that contribute to flightcrew error. Nor do adequate criteria, methods, and tools exist for designers and regulators to use to conduct such evaluations. To address some of deficiencies, this section focuses on issues and recommended changes that should be made in the criteria, standards, methods, and tools used in the design and certification processes.

Recent accidents, such as the Air Inter Airbus A320 accident near Strasbourg, provide evidence of deficiencies in design and certification. Although that accident highlighted weaknesses in several areas, it particularly highlighted the potential for apparently minor features or characteristics to play a significant role in an accident. In this instance, inadvertently setting an inappropriate vertical speed because of similarities in the way flight path angle and vertical speed are displayed on the FCU may have been an important factor in the accident. Although this issue was raised during the certification approval process, it was believed that the flight mode annunciations and PFD would compensate for any confusion caused by the FCU display, and that the flightcrew would use appropriate procedures to monitor the airplane's vertical path, terrain clearance, and energy state. This belief appears to have been incorrect.

Under current standards, potential flightcrew error and its consequences are not evaluated as extensively as flightcrew workload. The HF Team considers flightcrew error analysis (i.e., a process to find and eliminate predictable, design-induced error traps for flightcrews, and to 
identify consequences of flightcrew error) to be as fundamental to successful flight deck evaluation as workload analysis. Identifying designs that can induce flightcrew errors having undesirable consequences early in the design and certification processes would allow appropriate corrective action to be undertaken at a stage when cost and schedule pressures are less daunting. In addition to the A320 FCU design, other examples where flightcrew error analysis may have identified design features that have been implicated in serious incidents or accidents are: flightcrew awareness that the autopilot is approaching its control authority (B747 China Air over the Pacific Ocean ${ }^{16}$ ) and autopilot designs that allow pilot input to inadvertently create large out-of-trim conditions (A300-600 accident at Nagoya, Japan).

The HF Team identified many examples where automation interfaces are awkward and may be susceptible to flightcrew errors that have potentially undesirable consequences. One example frequently cited is the FMS interface. In some operational scenarios, the amount of headdown time required to operate the FMS is a major concern, because it can impair the flightcrew's ability to maintain flight path vigilance and traffic avoidance during critical phases of flight. Although there are many justifiable reasons for pilots to spend head-down time to use the FMS, undesirable reasons include difficulty in using the interface or difficulty in finding information in the FMS. Also, error messages like "invalid entry" for an entry format error do not help the flightcrew to understand and correct the error (Refer Recommendation AutomationMgt-5).

Other examples of interfaces that have the potential for flightcrew error are the mode selector panels which require the flightcrew to select the mode on one cockpit panel and refer to another cockpit area to confirm that the mode has actually been activated. As previously mentioned in this report, separation of the control (in this case, the mode selector control), from the display that shows the result of changing the control (the flight mode annunciation on the PFD), leaves the flightcrew vulnerable to misunderstanding which mode is active.

The FARs and associated advisory material have failed to keep up-to-date with current technology in many areas, including knowledge and awareness of human factors considerations. For example, most modern transport category airplanes have caution and warning systems that include distinct aural tones or other attention-getting sounds that complement the visual alerts as well as voice alerts for time critical warnings. Section 25.1322 currently addresses only visual alerting criteria, rather than the minimum standards that should be applied to modern transport category airplanes.

In consideration of the preceding discussion, the HF Team believes that appropriate criteria, standards, methods, and tools should be developed, including revising or updating existing material. Development of this material is necessary to provide design and certification personnel with the information necessary to allow them to identify and address areas where flight deck designs predictably produce flightcrew performance problems that can adversely affect safe flight.

\footnotetext{
${ }^{16}$ While this event did not involve a glass-cockpit airplane, the principle involved with autopilot awareness applies to the glass-cockpit airplanes within the HF Team's charter.
} 


\section{Recommendations}

The HF Team recommends consideration of new and revised regulatory criteria in some areas that have not kept pace with advancing technology and human factors knowledge. If new or updated regulatory criteria are adopted, there should be a parallel development of associated advisory material. The regulatory and advisory material must be written so that they can be practically applied by the applicant, and are clear about what the applicant must do, when the applicant is finished, and what criteria will be used to judge acceptance. The material should address acceptable processes that can be used, including methodology, rather than specific design requirements. The proper roles, safety objectives, and the relationships between associated airworthiness and operating rules must also be respected. In addition, we recommend that the standards and criteria be harmonized internationally.

\section{Recommendation Criteria-1}

\section{The FAA should require evaluation of flight deck designs for susceptibility to design- induced flightcrew errors and the consequences of those errors as part of the type certification process.}

\section{Discussion of Recommendation Criteria-1:}

As stated earlier, flightcrew errors occur for many reasons and have many potential contributing factors. It is impossible to prevent all human error without removing the human flexibility and adaptability that contributes significantly to safety. Moreover, it is the negative consequences of error we wish to eliminate, not necessarily the errors themselves. However, it is still desirable to minimize errors that are design or system induced. Therefore, as part of the certification process, the HF Team recommends that the FAA require evaluation of flight deck designs for susceptibility to design-induced flightcrew errors, and for the consequences of flightcrew errors that do occur. Flightcrew performance considerations such as workload and situation awareness should be evaluated for their contribution to error.

To implement this recommendation, we recommend that the FAA convene a working group with representatives from the Aircraft Certification Service, (including engineering, human factors, and flight test pilot expertise), Flight Standards Service, other airworthiness authorities (e.g., JAA), industry, and the research community to:

- Determine the acceptability of existing analysis tools and methods,

- Identify what changes should be made to existing standards and criteria, and take action to make those changes,

- Determine what new criteria and methods are needed, if any (we believe that some will be needed), and

- Recommend any appropriate research to develop tools and methods as needed. 
For existing regulations, several changes are already being considered to address human error. Currently, an Aviation Rulemaking Advisory Committee (ARAC) Harmonization Working Group (HWG) is considering revisions to $\S 25.1309$, including a proposal to require the consideration of flightcrew and maintenance errors. However, the analysis of human error is much broader than the scope of $\S 25.1309$. Therefore, the HF Team recommends that the $\S 25.1309 \mathrm{HWG}$ retain the existing regulatory language related to flightcrew error contained in $\S 25.1309$ and defer further regulatory changes in the area of flightcrew error to the new working group identified in this recommendation. Similarly, changes to $\S 25.1329$ are being considered to include human error concerns. We recommend that changes to existing regulations and development of any new regulations and advisory material be approached in an integrated fashion, rather than independently.

This will not be an easy recommendation to implement, since current methods may be inadequate to address all concerns. Further, the development and application of human error analysis methods and criteria acceptable to the FAA and industry may take a great deal of time and effort. However, the implementation of this recommendation should be started as soon as possible and it should be done in a consistent and integrated way with the implementation of Recommendation Processes-2.

\section{Recommendation Criteria-2}

The FAA should prepare and distribute interim guidance material that updates current autopilot certification policy.

\section{Discussion of Recommendation Criteria-2:}

Interim certification policy guidance is needed until current activity to update $\S 25.1329$, $\S 121.579$ and associated guidance material is complete.

Specifically, the HF Team believes that the following areas should be addressed by interim guidance:

- Pilot/autopilot interactions that create hazardous out-of-trim conditions;

- Autopilots that can produce hazardous energy states and may attempt maneuvers that would not normally be expected by a pilot; and

- Improved airplane flight manual wording regarding the capabilities and limitations of the autopilot. 


\section{Recommendation Criteria-3}

The FAA should task an appropriate Aviation Rulemaking Advisory Committee Harmonization Working Group (HWG) with updating the autopilot regulatory standards (14 CFR 25.1329). This HWG should include specialists knowledgeable in human factors methods and skills from both industry and the regulatory authorities.

\section{Discussion of Recommendation Criteria-3:}

The HF Team recommends that the AWO HWG be tasked with updating and revising $\S 25.1329$ and its associated advisory material to address multiple concerns and considerations. Specialists knowledgeable in human factors methods and skills from industry and the regulatory authorities should be added to the AWO HWG to help them effectively address the human performance concerns identified by the HF Team. Future rulemaking should address advances in technology and knowledge of human factors considerations, including:

- Envelope protection

- Intuitiveness (user friendliness)

- Autopilot mode complexity

- Flight mode annunciation

- Proliferation of autopilot modes

\section{Recommendation Criteria-4}

The FAA should revise/update the following specific FARs and associated advisory material:

\$ 25.1322 Warning, caution, and advisory lights: Revise to reflect the current and anticipated design practice for modern transport category airplanes.

\$ 25.1335 Flight Director: Revise to reflect the current and anticipated design practice for modern transport category airplanes.

$\$ 121.703$ Mechanical reliability reports: Revise the requirements to also include reporting of significant flight deck automation failures and/or anomalies that adversely affect safe flight path management. Reinforce the Aviation Rulemaking Advisory Committee (ARAC) activity in this area.

\section{Discussion of Recommendation Criteria-4:}

The FAA should task an existing or new working group to revise/update the FARs and associated guidance material listed in the recommendation. 
Section 25.1322: Current glass cockpit airplanes have sophisticated caution and warning systems (e.g., Engine Indicating and Crew Alerting Systems and Electronic Centralized Aircraft Monitoring). These systems incorporate distinct aural tones that complement the visual alerts as well as voice alerts for time critical warnings. Additionally, these systems include alerting and display priority logic and inhibit logic for certain phases of flight. The manner in which warning, caution, advisory, and procedural information is presented to the flightcrew is critical to safe operation. Section 25.1322 only addresses requirements for visual alerting criteria. The regulation should be revised to address the current and anticipated design practice for transport category airplanes, (i.e., aural tones, voice alerts, display priority logic, etc.).

Section 25.1335: Section 25.1335 requires the flight director system to provide a means to indicate to the flightcrew its current mode of operation. The regulation should be updated to require consideration of the interface between the flight director and autopilot. A minimum set of functional modes should be addressed, including performing basic airmanship tasks such as turns to a heading, climbing, descending, capturing an altitude, lateral and vertical navigation guidance, and envelope protection.

Section 121.703: Section 121.703 requires operators to report certain types of mechanical systems failures, inflight fires, and structural integrity problems. Significant flight deck automation failures or anomalies are not addressed (e.g., dual unrecoverable FMS resynchronization during oceanic flight, navigational display map shifts during critical phases of flight). These failures have potential negative safety impacts, but operators are not required to report such automation anomalies to the FAA. Reporting of significant automation failures or anomalies, in conjunction with other sources of information (e.g., ASRS reports) could help to identify potentially unsafe or undesirable design features. This information could also be used as an additional measure for assessing system safety (refer to the section on "Measurement of and Incentives for Safety"). 


\section{Knowledge and Skills of Designers, Pilots, Operators, Regulators, and Researchers}

“...reliance on automation and glass cockpit flying (especially long haul flights where pilots might only get 2 to 3 landings per month) causes a degradation in basic skills.”

"The pilot may be certified but is seriously lacking in actual automation skills."

"Crew training is insufficient to deal with all possibilities and modes of operation."

"...very few of the decision makers at the airline are sufficiently educated in flight deck automation issues.”

Representative comments from line pilots, source ALPA

The HF Team identified that, generally, the knowledge and skills of the people who make up the aviation system are excellent. However, we also identified some areas where the knowledge and skills are insufficient, especially with respect to human factors. While the findings below may not apply to each individual in the groups examined, the HF Team found these weaknesses for each of the groups as a whole. The following groups (or portions thereof) would benefit from improvement in the following knowledge and skill areas:

- Designers: Human-centered design principles, knowledge of the actual operating environment, human factors (beyond ergonomics), human performance (especially cognitive engineering) guidelines, methods, and research results;

- Pilots: Basic airmanship, unusual attitude recovery, CRM, team decision making, awareness of operational aspects of aircraft design philosophy, automation and mode management;

- Airline/operator management: Human performance considerations in areas such as flight deck automation, operational considerations related to the design philosophy of aircraft, design of procedures, checklists, manuals, and LOFT scenarios;

- Air traffic service personnel: Capabilities and limitations of FMS-equipped aircraft, line operations considerations;

- Regulators: Human performance evaluation methods, criteria, guidelines, and research results; identification of research requirements; operational knowledge about how the airplane will be flown; and

- Researchers: Operational, design, evaluation, and regulatory considerations that shape research needs and opportunities.

\section{Airplane Flight Deck Designers}


All manufacturers stated that they include human factors expertise on design teams, but the HF Team found that these HF specialists had varying amounts of authority and their participation was typically limited. Recently there has been an industry-wide growing awareness of the need to treat human factors as a valid discipline in the design for new systems (also see the section "Processes for Design, Regulatory, and Training Activities").

The HF Team found different definitions of "human factors specialist" being used within the aviation industry. In some cases, formal training or background in relevant areas was required (e.g., experimental psychology, industrial engineering, human/computer interaction), and in other cases, human factors expertise was related primarily to experience in piloting and flight test.

The HF Team also found that, in some cases, design decisions appear to be based on an engineering design perspective, rather than how a flightcrew will use a system in an operational environment. For example, the mode definitions on certain airplanes appear to be more intuitive to a controls engineer designing a system with specific and limited concerns in mind than to a pilot operating the system. This is likely to be a contributing factor to the difficulty pilots have in understanding the autoflight modes. Likewise, pilots express numerous concerns about the difficulty in using current flight management systems, and they often mention that these systems appear to be designed without considering important flightcrew operational needs, which leads to an increased potential for flightcrew errors.

In yet other cases, the human factors aspects considered in the design were primarily ergonomic considerations (e.g., physical layout, reachability of controls, legibility). While ergonomic considerations are certainly necessary, they are not the only human factors issues that should be considered. The cognitive requirements of the flight deck tasks and functions often are not considered adequately or explicitly, and system designers who do not have human factors skills may not find optimum or even adequate design solutions. For example, consider the "undo" function available for modern personal computer systems. This capability is often not available to pilots using modern FMSs. The advantages of such features are well known, but frequently are not included in modern flight deck designs. There are several reasons why such features are not included, many related to cost, but a contributing factor is insufficient knowledge about how to design systems to be human-centered.

The Team found that none of the four airframe manufacturers or the avionics manufacturer that we visited distributed a comprehensive, written set of human-centered design principles to their design teams for use in the design process. ${ }^{17}$ All the manufacturers use design principles, as embodied in their flight deck designs. The concern is that these principles are sometimes implicit, rather than commonly understood, agreed upon, and applied consistently by a flight deck design team.

\footnotetext{
${ }^{17}$ It should be noted that, since our visit, two airframe manufacturers have written a set of design principles and distributed them publicly.
} 
In some cases, designers do not know or use research and technology results related to human performance, because they neither know where to find such results, nor have they been educated or trained to use them, or the results are not in a form they can directly apply. This is also a communication and coordination issue; see the section "Communication and Coordination" for related discussion.

\section{Pilots}

Based on the incident data, accident data, and pilot and operator input evaluated by the HF Team, we have concerns about pilot basic airmanship skills and general airmanship knowledge in several areas. One area is the degradation of manual flying skills of pilots who use automation frequently, or who participate in long-haul operations, and therefore do not have the opportunity to perform manual takeoffs and landings more than a few times a month. It is also rare for pilots to experience the edges of the flight envelope, or receive training on special issues such as high altitude stability and handling qualities. Yet there have been incidents in both the MD-11 and the A300-600 of high-altitude upsets where the autopilot disengaged for various reasons, including turbulence, resulting in pilots taking over control of an out-of-trim aircraft in a flight regime with which they were not very familiar.

A second area of concern is in the skills needed to perform recovery from unusual aircraft attitudes. Pilots at many airlines are not required to perform recoveries from most types of unusual attitudes in training or on checkrides. While a significant number of pilots have a military background where they were trained or have experience with acrobatic maneuvers, it is less common to have such a background than it used to be. In many cases, even former military pilots have not performed such maneuvers for a long time. Yet inadequate response to unusual attitudes has been implicated as a possible contributing factor in several accidents (ATR-72 crash near Roselawn, possibly the B737 accidents near Colorado Springs and Pittsburgh). There is enough concern in the aviation community that the National Transportation Safety Board (NTSB) has made several recommendations regarding training in recovery from unusual attitudes. Several airlines and organizations such as Flight Safety International have instituted advanced maneuver training, including unusual attitude recovery, and the FAA has issued Flight Standards Handbook Bulletin for Air Transportation Number HBAT 95-10 providing guidance for Selected Event Training (voluntary flight training in hazardous inflight situations not specifically identified in FAA regulations or directives). The HF Team endorses this trend.

Yet another concern is in the flightcrew management of the flight deck and its automated systems. The previous discussions of issues with situation awareness (see the section "Flightcrew Situation Awareness") and management and direction of automation ("Flightcrew Management and Direction of Automation") make it clear that management of the flight deck is a fundamental skill area for flightcrews of modern transport aircraft. It was suggested to the HF Team that the notion of basic airmanship should be expanded to explicitly include management of the flight deck systems, including the automation -- and we agree. Flightcrews should explicitly receive instruction and practice in when and how to:

(1) appropriately use automation; 
(2) transition between various levels of automation,; and

(3) revert to manual flight.

Other important knowledge and skill areas for flightcrews are flightcrew resource management (already underway in many airline training programs), understanding of decisionmaking processes (including team decision making and handling unanticipated events), workload and attention management, and understanding of other human cognitive processes (especially cognitive biases and limitations as they apply to flightcrew problem solving in airline operations). While excellent examples of training programs incorporating these concepts can be found, some airlines still do not adequately address CRM, especially with respect to the use of meaningful LOFT scenarios. This is especially true for instances where LOFT scenarios do not accommodate the operating environment in which flightcrews may experience difficulty (e.g., oceanic operations, international operations, adverse weather scenarios, etc.), or where LOFT scenarios do not effectively use the time allocated.

A primary mechanism for flightcrews to gain knowledge and skills, is, of course, the operators' training programs. Yet the operators often believe that training must be focused on ensuring that the flightcrews pass their checkrides, and that the checkride criteria do not include or emphasize some of the skill areas mentioned above, such as management of automation or other known problem areas of line operation. Moreover, checkrides often test for maneuvers that are not considered to be as important as the skills mentioned above, or are maneuvers performed on a frequent basis in line operations (e.g., Instrument Landing System (ILS) approaches, autoland, etc.) that may not serve as the best use of training or evaluation time. In addition, the maneuvers included in checkrides should be evaluated for continued relevance, be phased out, or be conducted in a manner that reflects the way they could be encountered in unusual circumstances during line operations (e.g., stalls, steep turns).

Based on inputs from pilots and airline training departments, the emphasis of checkrides and the criteria used no longer necessarily reflect the best balance of knowledge and skills needed to safely conduct line operations. AQP is a move in the positive direction of encouraging more line-oriented training and evaluation as appropriate, and of encouraging inclusion of CRM in training programs. The HF Team strongly supports this trend with the qualification identified below.

There appears to be continuous and intense economic pressure to decrease (or at least not increase) the time required for training. In fact, one of the incentives for the airlines to incorporate $\mathrm{AQP}$ is the potential for increased time periods between required recurrent training. The HF Team supports the intent of AQP and updating of FAR 121 Subparts N and $\mathrm{O}$ to improve the efficiency of training, but is concerned about any decrease in investment in pilot expertise. The HF Team is concerned that the economic benefits of AQP may be receiving emphasis over the need for enhancing safety. A careful re-examination of the balance among content, length, and type of training is needed (also see the section "Processes for Design, Regulatory, and Training Activities"). This re-examination should be done to assure that each qualification program covers important skills needed for line operations (e.g. basic 
airmanship, management of automation) and minimizes the repetition of maneuvers or skills successfully demonstrated in day-to-day operations.

\section{Airline/Operator Management}

The Team found that decision makers in operators' organizations sometimes appear to be concerned with short term costs with regard to flightcrew training and equipment purchases, rather than being sufficiently sensitive to the long-term need to invest in building or enhancing flightcrew skills and knowledge and updating flight deck equipment. Also, because safety benefits may sometimes be difficult to see or quantify, concerns over costs tend to take priority in decisions. An example of where this concern is perceived to affect decisions about equipment purchases is in the choice of whether to buy flight deck system enhancements offered by the manufacturers (also see the Section "Measurement of and Incentives for Safety").

An area where management is perceived to lack knowledge or commitment is in identifying for line pilots the flight deck design philosophies for the aircraft in their fleet. Many airlines have reduced their engineering or operations departments to the point that meaningful contributions to flight deck design philosophy during acquisition of new aircraft is limited. As mentioned previously, the airline flightcrews do not get trained in the design philosophy, yet understanding the operational assumptions embodied in the design could reduce the potential for automation surprises. Of course, as mentioned above, the airplane manufacturers have not explicitly communicated and distributed their design philosophy. Rather, at least until recently, the operator had no choice but to infer the philosophy from its implementation in the flight deck.

Training department managers and other appropriate management should be aware of these design philosophies as they relate to operational use and how they relate to the operator's philosophy on using automation. This information affects the content of training programs and manuals and the design of procedures. Ideally, the operators would work closely with the airplane manufacturers, so that the operations philosophy and the flight deck design philosophy are consistent and compatible.

\section{Air Traffic Service personnel}

The HF Team heard numerous concerns from pilots and airlines that air traffic controllers and other air traffic service personnel are not sufficiently knowledgeable about modern aircraft or seemed to misunderstand or ignore the capabilities, limitations, operational procedures, and constraints of FMS or autoflight system equipped aircraft. ATS clearances and procedures have not been kept current with flight deck evolution. FMS-friendly procedures or clearances are lacking, even though the same air traffic objectives could be easily achieved by taking full advantage of the capabilities of highly automated aircraft (e.g., use of direct routing to an intercept waypoint versus a vector to intercept and track outbound on a navigation aid radial). 


\section{Regulators}

FAA regulatory personnel would benefit significantly from greater knowledge and expertise in human factors and, in some cases, from increased operational and technical knowledge about the airplane types for which they are responsible. Certification of modern automated aircraft and evaluations of flightcrews increasingly involve considerations related to the interaction between human(s) and machine(s). Members of teams who conduct certification evaluations, such as flight test pilots, inspectors, Aircrew Program Managers and ACO engineers, are not necessarily trained human factors specialists, nor is human factors expertise necessarily part of these teams. This lack of training and expertise contributes to insufficient quality and inconsistent regulatory results in the certification process with respect to flightcrew performance issues.

- Certification flight test pilots. Certification flight test pilots bear a large share of the responsibility for providing the flightcrew perspective during the flight deck certification process. Because of the lack of objective criteria and methods (or lack of knowledge of the methods and criteria that do exist) they must often base their assessment on subjective evaluation of the displays, controls, and system operation. While it is true that their subjective evaluation generally reflects good judgment, it does not represent an objective, systematic evaluation of human performance for the target user population (i.e., the "typical" line pilot), nor does it always address the operational environment expected in service. Because most of these pilots are highly experienced, the results are generally very good. However, because experience varies, the results of the certification process may also vary. We heard from manufacturers that they saw differences and inconsistencies in certification results, depending on who was making the decision (also see the section on "Processes for Design, Regulatory, and Training Activities").

In addition, the flight test pilots do not always evaluate some important aspects of flight deck operation from the perspective of a line flightcrew. They may evaluate operation for a pre-defined high workload situation, such as one pilot incapacitated, but may not necessarily consider effects of pilot-flying/pilot-not-flying coordination used in service (e.g., monitored approach, International Relief Officer duties, etc.). As a result, designs are not always evaluated for flightcrew coordination in the operational environment in which they will be used.

- Aircraft Evaluation Group (AEG) pilots. Adequate training is not always available for the operational evaluations that these pilots are required to perform. For example, AEG pilots routinely are asked to provide operational judgments on characteristics such as nonnormal procedures and related handling qualities when new or modified aircraft are proposed for U.S. airline operations under Part 121. Yet, these same pilots may not have been trained or type rated in the airplane types for which they are responsible. In addition, they may be lacking in recent experience on those types for which they are rated or they

\footnotetext{
${ }^{18}$ Notice of Proposed Rulemaking 94-35, Docket No. 27993.
} 
may not have experience with the aircraft in an operating environment. Also, the AEG pilots have skill needs in human factors similar to the certification test pilots.

- Other Flight Standards personnel._The level of relevant technical expertise of inspector personnel in many Flight Standards District Offices (FSDOs) is generally insufficient and has been decreasing relative to levels historically achieved. Unlike most authorities' pilots worldwide, FAA inspectors no longer operationally fly in line operations even though regulatory authority exists for gaining valuable experience in this manner. In addition, inspectors may lack some specific relevant skills. For example, inspectors may not have experience in the class of aircraft for which they are responsible, such as glass-cockpit airplanes, or they may not be familiar with operations (e.g., oceanic) for which they have responsibility. This may adversely affect their ability to assess and apply human performance considerations, even if HF training was provided. For example, they may inappropriately apply assumptions about older airplanes to highly automated airplanes.

In addition, regulators often do not know about or use research and technology results related to human performance, because they neither know where to find such results, nor have they been educated or trained to use them, or the results are not in a form they can directly apply. As with designers, this is a communication and coordination issue, as well as a knowledge and skills issue associated with insufficient training.

\section{Researchers}

Just as designers and regulators lack experience and knowledge with research results, researchers are often unaware of the needs and constraints of the operational and certification community. As discussed in the section on "Communication and Coordination," researchers need to seek out such information, and regulators and designers need to clearly describe their needs and requirements, and follow up research efforts to help assure its relevance and eventual use. 


\section{Recommendations}

\section{Recommendation Knowledge-1}

The FAA should encourage flight deck design organizations to:

(1) Make human factors engineering a core discipline of the flight deck system design activity, and

(2) Ensure that the design team has sufficient human factors and operational knowledge and expertise by:

- Distributing guiding principles for flightcrew-centered design (as described in Recommendation Processes-1) to all design team members;

- Including human factors expertise as part of the design team;

- Assuring that each relevant member of the team has at least a basic knowledge of human factors, in order to understand and communicate human performance issues and human-centered design considerations; and

- Assuring that flight deck design team members have relevant operational knowledge.

\section{Discussion of Recommendation Knowledge-1:}

Organizations responsible for overall flight deck design or the design of systems used in a flight deck should make certain that design teams have appropriate expertise and knowledge of human factors. The human-centered design principles of the organization (or of the flight deck in which the system will be integrated) should be explicit and distributed to the design team members. Individuals with human factors expertise should be a fundamental part of the design team, just as human factors should be a core discipline of the design activity. The design team members who are not human factors specialists should be sufficiently trained in human factors to understand and communicate human performance issues and design considerations. All relevant design team members should be provided access to the human factors literature and existing guidelines in a way that makes the information accessible and easy to use.

The design team members should have sufficient operational knowledge to incorporate considerations of the operational environment into the design and evaluation process. Suggested ways of accomplishing this would be to encourage or require regular jumpseat observation (even more than is now done), encourage more interaction with a range of pilots who have recent, representative line experience, and include structured evaluations by operators or line pilots during the design process. 


\section{Recommendation Knowledge-2}

The FAA should reassess the requirements that determine the content, length, and type of initial and recurrent flightcrew training. Ensure that the content appropriately includes:

- Management and use of automation, including mental models of the automation and moving between levels of automation;

- Flightcrew situation awareness, including mode and automation awareness;

- Basic airmanship;

- Crew Resource Management;

- Decision making, including unanticipated event training;

- Examples of specific difficulties encountered either in service or in training; and

- Workload management (task management).

The FAA should work with industry to develop guiding principles and associated advisory material for training, operational procedures, and flightcrew qualification for the areas listed above.

\section{Discussion of Recommendation Knowledge-2:}

It may or may not be necessary to increase the amount or length of initial or recurrent training provided to flightcrews, but the balance among the content, length, and type of training should be re-examined in light of lessons learned about how flightcrews interact with modern transport airplane flight decks. The content, length, and type of training should suitably reflect the philosophy and features of the particular airplane design.

Content of initial and recurrent training: The content of training should include a large set of regularly updated, realistic LOFT scenarios, regular updates on specific in-service/training difficulties and other relevant in-service experience, and available safety information (e.g., the Takeoff Safety, CFIT, and Windshear Training Aids). In addition, the FAA should require operators' initial and recurrent training programs to address:

- Management and use of automation

See Recommendations AutomationMgt-1 and AutomationMgt-2 for specific items to be covered under this topic.

- Flightcrew situation awareness

See Recommendation SA-2. 
- Basic airmanship

This area should cover skill degradation and advanced maneuvers training, including unusual attitude recovery, high altitude handling qualities, full stalls, ${ }^{19}$ engine failure immediately after takeoff or during approach, minimum control speeds (see

Recommendation Knowledge-3). It should also include the idea that basic airmanship in advanced flight decks requires the flightcrew to be flight deck managers, involving workload management. While this has always been an element of good piloting, it is even more critical in advanced flight decks.

\section{- Crew Resource Management}

Many airlines are already doing an excellent job of incorporating CRM into their training curricula; this should be reinforced and encouraged in all operators. The HF Team supports the proposed regulatory requirement for CRM training by all Part 121 operators and applicable Part 135 operators.

- Decision making, including unanticipated event training

This area should cover general decision making skills and fundamentals, including team decision making and cognitive biases, as an important part of the content of a training curriculum. Flightcrews also should be trained in dealing with unanticipated events, (e.g., subsystem failures not covered by checklists) and how to use multiple checklists, especially during high workload conditions.

- Examples of specific difficulties encountered either in service or in training

See Recommendation Comm/Coord-4 for items that should be covered under this topic.

Length of training: In addition to assuring that the length of the training is sufficient to cover the appropriate content, the FAA should require the length of training to be based on the need applicable to a given airplane type, recognizing that length may vary for different airplane types. It is not necessarily appropriate for the training time to be equivalent for different flight deck designs and different automation philosophies.

Moreover, the training should also be adapted to the background of the pilot (e.g., glass vs. non-glass experience). As an example of one activity at one airline to address this issue, Delta Airlines has produced a training video designed to help pilots transitioning from non-glasscockpit airplanes to glass-cockpit airplanes. The HF Team endorses the trend to tailor the training to fit the background of flightcrews.

Type of training: A variety of training tools and methods should be considered for long-term expansion of flightcrew knowledge, including personal computer-based training tools as an alternative, inexpensive means of providing information and encouraging exploratory learning

\footnotetext{
${ }^{19}$ The HF Team intends this recommendation to include training for full stalls in simulation, and the simulation models must realistically reflect the actual airplane behavior to be effective for training. The training of full stalls in actual flight is not necessarily appropriate.
} 
by flightcrews. In addition, the FAA and the airplane operators should explore incentives to encourage practicing and training beyond the minimum required. See Recommendation Processes-3 for related discussion.

This assessment and redefinition of content, length, and type of training should be accomplished as soon as possible, and should be based on a set of guiding principles. Principles advocated by the HF Team include:

- Assure consistency of flightcrew qualification internationally, to the extent possible.

- Not all aircraft are equal - be ready to increase the length and emphasis on training when there is a large difference between the flight deck with which the pilot is familiar and the one for which the pilot is being trained.

- Better match Standard Operating Procedures and flightcrew qualification assessment with basic type design philosophy.

The HF Team supports the intentions behind AQP, such as training to proficiency, training and evaluation as a flightcrew, etc. We agree that it is important to avoid training the flightcrews to perform in a rote manner; rather, encourage them to understand the underlying principles behind the system design (see related Recommendation SA-2). However, the HF Team believes that care must be taken to assure that AQP advantages are applied to safety improvements where vulnerabilities still exist, rather than exclusively to economic advantage (e.g., single visit, lengthened evaluation periods, etc.).

The guiding principles and associated advisory material called for in this recommendation should be explicitly written down, distributed to appropriate organizations, and reflected in training courses, training processes, and operational procedures.

\section{Recommendation Knowledge-3}

The FAA should strongly encourage or provide incentives to make advanced maneuvers training an integral part of the training curriculum, especially in recurrent training.

Discussion of Recommendation Knowledge-3:

We recognize that several operators are already conducting this training, and the FAA has issued a bulletin containing guidance for implementing advanced maneuvers into a training program. We strongly support those actions and consider this to be a sufficiently important issue to have a separate recommendation to reinforce its implementation.

Incentives could include alternate means of addressing certain required maneuvers if the objective is obtained through the advanced maneuvers training. For example, if the advanced maneuvers training includes high bank angle recovery and full stalls, the traditional requirement for steep turns and approach to stall maneuvers could be considered to be completed. 
The HF Team's assumption is that most, if not all of this training would be done in training simulators. We are not advocating, for example, that line pilots perform full stalls in the airplane. These maneuvers could be done in a high-fidelity simulator.

Insufficient simulator fidelity might be an issue in implementing this recommendation. For example, the aerodynamic models of many simulators do not accurately reflect the behavior of the aircraft under all the desired conditions. However, it is desirable for line flightcrews to be exposed to as much of the flight envelope as possible so that in unusual circumstances, it is probable that at least one flightcrew member has relevant background or training and can make constructive contributions to detecting and resolving the unusual situation.

\section{Recommendation Knowledge-4}

The FAA should reassess recency requirements for flightcrews involved in long haul operations. Consider providing incentives and alternative methods for flightcrews to practice takeoffs and landings, and perhaps arrival and departure procedures that are infrequently used.

\section{Discussion of Recommendation Knowledge-4:}

Primary responsibility for implementing this recommendation lies with the FAA Flight Standards Service, working with operators. In assessing the recency requirements, they should consider the use of automation (i.e., how many operations are done manually versus using autopilot).

\section{Recommendation Knowledge-5}

The FAA should reassess the airman certification criteria to ensure that pilots are released with a satisfactory level of skills for managing and using automation. Since current training is often oriented toward preparing pilots for checkrides, the airman certification criteria should be reassessed to ensure appropriate coverage of the topics listed in Recommendation Knowledge-2.

\section{Discussion of Recommendation Knowledge-5:}

Airman certification criteria should be redefined so that release of flightcrew members to the line only occurs when they demonstrate satisfactory skills in managing and using the automation (also see Recommendation SA-2), rather than expecting them to learn these skills during line operations. Correspondingly, initial and recurrent qualification courses should be oriented to support these criteria. In addition, the use of LOFT scenarios in these courses should reinforce demonstration of key automation skills. 


\section{Recommendation Knowledge- 6}

Operators should ensure that flight safety and training managers are appropriately educated about human factors considerations, particularly with regard to automation.

Discussion of Recommendation Knowledge-6:

Pertinent managers should be informed about human factors considerations, especially those managers responsible for procedures, manuals, and training program design. Managers in technical organizations making decisions about airplane purchases should be informed about automation design for the different aircraft, and its potential effects on flightcrew qualification, training, and operations. The FAA should encourage this education process.

\section{Recommendation Knowledge-7}

The FAA should improve the education of Air Traffic Service personnel about the capabilities and limitations of highly automated airplanes.

Discussion of Recommendation Knowledge-7:

FAA Air Traffic Services should work with operators to improve training and/or familiarization mechanisms for air traffic service personnel to better understand what the flightcrews of highly automated aircraft must do to accommodate ATS procedures. For example, jumpseat observations are already permitted for air traffic personnel; this participation should be more strongly focused towards helping relevant ATS personnel understand the capabilities of glass-cockpit airplanes in the specific environment intended.

\section{Recommendation Knowledge-8}

The FAA should provide appropriate regulatory personnel with a guide or roadmap to current Federal Aviation Regulations, advisory material, policy memoranda, and other guidance material dealing with human performance related to the flightcrew-vehicle interface. The FAA should ensure that this material is used in aircraft certification projects, airline qualification program assessments, and airman qualification.

\section{Discussion of Recommendation Knowledge-8:}

As a first step towards increasing the knowledge of certification personnel in the area of human factors, the Aircraft Certification and Flight Standards Services should specifically identify currently existing regulatory tools and criteria related to human factors. These tools include the FAR, the Joint Aviation Requirements (JAR), and associated guidance material (e.g., ACs, JAA advisory material, policy memoranda). A synopsis of these tools and criteria 
should be provided to ACOs, AEGs, and FSDOs as guidance for use in emphasizing humanfactors-related reviews of existing or planned projects.

\section{Recommendation Knowledge-9}

The FAA should develop a systematic training program for appropriate Aircraft Certification and Flight Standards Services personnel to provide initial and recurrent training in the area of human factors as it relates to certifying new products and evaluating flightcrew performance. The training should include instruction on:

- Insight into the relationship among the flightcrew, the flight deck design, and the operational environment;

- Flightcrew information processing;

- Workload, human error, and situation awareness;

- Other flightcrew performance issues, including fatigue, CRM, and attention management;

- Design and evaluation of flight deck displays;

- Aircraft control laws and feedback systems;

- Human-automation interaction;

- Human-centered design principles and guidelines; and

- Ergonomics -- fitting the design to the user.

\section{Discussion of Recommendation Knowledge-9:}

The training program should be regularly updated and periodic refresher training should be provided. A program of continuing education to provide awareness of the current status and ongoing work/progress in this area should also be developed, along with methods for distribution. This training should include relevant human factors theory and practical applications, as well as guidance to resources (especially people) to help resolve human factors issues in the evaluation process. The training program should give regulatory personnel a solid foundation on which to ensure that HF concerns are understood during the regulatory process.

As regulations and policy/guidance material are created and updated with regard to human factors, the need for regulatory personnel awareness and training becomes even more critical. This training should be required for FAA flight test pilots, flight test engineers, and certification personnel involved in the approval of systems affecting the flightcrew interface, and appropriate Flight Standards personnel (e.g., AEGs, FSB members). 


\section{Recommendation Knowledge-10}

The FAA should appropriately staff the standards organizations and aircraft certification offices with human factors expertise and integrate personnel with such expertise into certification teams, participating and applying their expertise in the same manner as other certification team members (e.g., airframe, flight test, systems and equipment, propulsion).

Discussion of Recommendation Knowledge-10:

One way to implement this recommendation would be for the Aircraft Certification Service to appropriately staff the standards organizations and ACOs with human factors expertise. The roles and responsibilities of the FAA human factors specialists should include:

- Review, critique, and assess the manufacturer's flight-deck-related certification plans;

- Participate in selected development activities to assure adequacy of the design;

- Review and assess flight deck relevant reports of tests and analyses submitted by manufacturers;

- Participate in development of flight deck certification requirements;

- Participate in certification testing; and

- Define criteria and performance measures.

\section{Recommendation Knowledge-11}

The FAA should increase Aircraft Certification and Flight Standards Services personnel's knowledge about each other's roles and responsibilities. In particular, increase certification pilots' and engineers' knowledge of line operations considerations, and Aircraft Evaluation Group personnel's knowledge about airworthiness certification considerations.

Discussion of Recommendation Knowledge-11:

Recommended ways to accomplish the objectives of this recommendation include developing guidelines and necessary procedures to:

- Expand the jumpseat authority for certification test pilots to improve their knowledge of line operations, possibly to include permitting certification test pilots and selected Flight Standards pilots (e.g., AEG pilots) to serve as flightcrew members in actual line operations. This area was considered to be extremely important by the team members. 
- Assign appropriate ACO engineers to temporary duty at Certificate Management Offices (CMO) for the purpose of exposing them to airline operations and Flight Standards duties. As part of this assignment, ACO personnel should be given temporary cockpit jumpseat authority so they could observe first hand the day-to-day operations of an airline from the flightcrew's point of view.

- Assign Flight Standards personnel from AEGs and CMOs to temporary duty at an appropriate ACO. The purpose would be to expose these personnel to certification duties.

\section{Recommendation Knowledge-12}

The FAA should improve the knowledge of personnel in Aircraft Certification and Flight Standards Services about processes for identifying and communicating requirements for research (either specific studies required or identification of areas of concern).

Discussion of Recommendation Knowledge-12:

The FAA should coordinate and provide documentation to each of their personnel about the process for identifying and communicating requirements for research. It will require more than documentation to succeed in fostering this communication, however. Significant guidance and encouragement from appropriate management personnel will be necessary. (See related Recommendation Comm/Coord-8.)

\section{Recommendation Knowledge-13}

The FAA should encourage researchers to learn more about industry and FAA's research needs and about operational considerations in aviation.

\section{Discussion of Knowledge-13:}

The research community consists of many types of research labs in a variety of locations, including the FAA, NASA, other government research laboratories, universities, and industry. Each organization will require its own type of education. There are several ways to implement this recommendation. For example, in many instances, researchers addressing flight deck problems should have exposure to aircraft flight deck operations. To the extent practical and feasible, the FAA and airlines should facilitate simulator or jumpseat observation. As discussed in Recommendation Comm/Coord-8, communication with operators and airframe and avionics manufacturers should be encouraged so that researchers learn more about their needs. Certification and flight standards management should foster the processes of communicating research needs, and provide guidance for doing so. 


\section{Cultural and Language Differences}

\section{Culture}

Culture consists of the norms, attitudes, values, and practices that members of a nation, organization, profession, or other group of people share. In a survey of pilot attitudes towards automation, very large national cultural differences were found..$^{20}$ The observed differences do not imply that one national culture is superior to another or that there are "culturally correct" attitudes regarding automation. Rather, they suggest that the automated systems reflect the culture in which they were designed and that these systems may be received and used quite differently in other cultures.

Two dimensions of culture that have been isolated in research have been found to affect the way flightcrews manage automation and accept and practice concepts such CRM. ${ }^{21}$,22 Powerdistance refers to the nature of relations between leaders and subordinates, where subordinates in high power-distance cultures tend to accept and expect autocratic leadership and are generally unwilling to question the acts or decisions of leaders. Individualism-Collectivism reflects the extent to which an individual's behavior is defined and influenced by others. Both of these dimensions represent a perspective with which to view the way flightcrews interact with automation, which they may regard as an electronic crewmember.

A specific area where observed national cultural differences may have an effect is whether the pilots will turn the automation on or off (or increase/decrease the level of automation) when they are confronted by non-normal situations. A related issue is whether flightcrews will disengage the automation instead of reprogramming when changes in the flight path are desired (such as an approach or runway change) under high workload conditions, or when the flightcrew members are confused by or unable to get desired results from the autoflight system. For example, while $52 \%$ of the pilots surveyed agreed that programming the FMS should be avoided under high workload conditions, the variation across national cultures was between $35 \%$ and $64 \%$. There was an even larger difference among national cultures in the perception that the operator's organization expects flightcrews to always use automation, varying from $32 \%$ to $84 \%$ agreement. ${ }^{23}$ Consistent with these survey results, we found from our meetings with airlines and pilots that different operators have different approaches to the

\footnotetext{
${ }^{20}$ Sherman, PJ \& Helmreich, RL. (1995). Attitudes toward automation: The effect of national culture. In Proceedings of the Eighth International Symposium on Aviation Psychology (pp. 682-687). Columbus, OH: Ohio State University.

${ }^{21}$ Hofstede, G. (1980). Culture's consequences: International differences in work-related values. Beverly Hills, CA: Sage.

${ }^{22}$ Merritt, AC \& Helmreich, RL. (1996). Human factors on the flight deck: The influence of national culture. Journal of Cross Cultural Psychology. 27, 6-25.

${ }^{23}$ Helmreich, RL, Merritt, AC, and Sherman, PJ (1996). The Flight Management Attitudes Questionnaire: An international survey of pilot attitudes regarding cockpit management and automation. Study in progress. Austin, Texas: NASA/University of Texas/FAA Aerospace Crew Research Project.
} 
use of automation and that their training and procedures reflect these differences. These differences are undoubtedly influenced by both organizational characteristics and culture.

Given the evidence that culture does influence flightcrew members' use of automation, the pilot model used by regulatory officials to represent the range of expected pilot behaviors should take these cultural effects into consideration. (See the Section "Flightcrew Management and Direction of Automation" for other discussion regarding flightcrew use of automation, and its evaluation by certification and operational specialists.) Similarly, the autoflight system designers' assumptions should be consistent with how the pilots will use the automation or there is an increased potential for flightcrew error. Technical specialists at the manufacturers expressed surprise at some ways that automation is used in line operations, indicating a less-than-complete understanding of operating practices.

Organizations may develop unique cultures, just as nations do. Organizational cultures generally reflect national culture, but the history, practices, and management of organizations are also factors. In addition, there can be distinct subcultures within organizations. For example, in terms of both attitudes regarding automation and acceptance of CRM concepts, significant differences have been found among the different airplane fleets of individual operators. ${ }^{24}, 25$ The management of particular fleets influences the norms of these subcultures within the operator's organization. While the HF Team did not extensively explore this issue, the influence of organizational leadership and culture, operations management, and corporate culture have been recognized as important factors in the flightcrew's approach to operating the aircraft. ${ }^{26}$

Yet another cultural factor that may influence automation usage is age. The HF Team heard several comments about how younger pilots tend to be more comfortable with the automation and programming the FMS, sometimes preferring to use a higher level of automation than may be considered appropriate for the circumstances. We even heard some younger pilots being referred to as "Nintendo kids." While it is important to understand the potential influence of this factor, it is important not to overgeneralize the issue of age, because many exceptions to the generalization exist.

These are only a few of the possible cultural factors that influence the coordination between the flightcrew and the flight deck. The HF Team assessment of this area was very limited, but was enough to recognize that cultural effects can be important -- and at present, we do not understand as much as we should about their effects.

\footnotetext{
24 ibid.

${ }^{25}$ Helmreich, RL \& Foushee, HC (1993). Why Crew Resource Management? Empirical and theoretical bases of human factors training in aviation. In EL Wiener, BG Kanki, and RL Helmreich (Eds.), Cockpit Resource Management (pp. 3 - 45). San Diego: Academic Press.

${ }^{26}$ Proceedings of the FAA Office of System Safety Workshop on Flight Crew Accident and Incident Human Factors. June 21-23, 1995.
} 


\section{Language}

An issue related to but distinct from culture is that of language. English is the standard language of aviation, and airplane systems interfaces, manuals, procedures, and interactions with ATS are usually in English (although there are some exceptions). The HF Team heard concerns related to the use of these systems, documents, and procedures by pilots and controllers for whom English is not their native language, and who had varying levels of competency in English.

In general, concerns were raised that misunderstandings may occur when non-native English speakers must use English. Several examples where these misunderstandings may occur include:

- Situations involving a mix of native and non-native English speakers, among flightcrew members on the flight deck and between flightcrews and controllers;

- English-language-based flight decks operated by flightcrew members whose native language is not English. This is exacerbated by abbreviations and cryptic messages on caution and warning systems, flight mode annunciators, control display units, etc.; and

- Non-English-based flight decks (e.g., Russian aircraft based on the Cyrillic alphabet) being operated by flightcrew members whose native language is not the same as the one on which the flight deck is based.

Using ICAO standard phraseology may help reduce the potential for miscommunications. However, the ICAO phraseology standards are outdated for many new types of operations now occurring in the aviation environment (e.g., sidestep approaches, converging approaches, FMS approaches, RNP).

\section{Recommendations}

\section{Recommendation Culture-1}

The FAA should ensure that research is conducted to characterize cultural effects and provide better methods to adapt design, training, publications, and operational procedures to different cultures. The results of the research should also be used to identify significant vulnerabilities, if any, in existing flight deck designs, training, or operations, and how those vulnerabilities should be addressed.

\section{Discussion of Recommendation Culture-1:}

Understanding of the problems and issues in this area is limited but growing. There is a need to accelerate that growth, and proceed with implementation of methods to incorporate the 
knowledge. The results of research in this area must be transitioned to the target customer community as soon as possible.

There may be resistance to this recommendation by organizations who believe their culture or practices are under question.

\section{Recommendation Culture-2}

The FAA should encourage simplified flight deck messages, training, manuals, and procedures with clearer meaning to non-native English speakers. The FAA should encourage the use of internationally understood visual symbols and pictures where appropriate, rather than verbal descriptions or directions.

\section{Discussion of Recommendation Culture-2:}

The FAA should work together with industry to implement this recommendation. They should encourage the use of simplified messages on caution and warning systems, and corresponding clear and simple procedures.

\section{Recommendation Culture-3}

The FAA should provide leadership to update ICAO phraseology standards and to encourage their use.

Discussion of Recommendation Culture-3:

The FAA should work with ICAO to assure that the ICAO phraseology is updated where appropriate, especially for new types of navigation procedures and approaches. The updated phraseology should be adopted for standard use to reduce the potential for miscommunications. ICAO phraseology that does not need updating should be adopted for standard use as soon as possible without waiting for the updates to be developed.

\section{Recommendation Culture-4}

The FAA should promote timely and clear communications between flightcrews and Air Traffic Services through:

- Accelerated efforts for transmission of information via datalink, as appropriate (e.g., Automatic Terminal Information Service (ATIS), weather, pre-departure clearances (PDC));

- Assuring clear and intelligible transmission of ATIS and clearance information where datalink is unavailable or unsuitable; and

- Standard procedures and taxi routes. 


\section{Discussion of Recommendation Culture-4:}

The FAA should encourage the transmission of ATIS, weather, PDC, and other appropriate information by datalink. The FAA should also assure that ATIS and clearance information is communicated clearly and intelligibly from Air Traffic Services; that is, the transmission of information should be done slowly and distinctly enough for comprehension by all flightcrews, including those whose native language is not English. In addition, the FAA should encourage the use of standard taxi routes to facilitate clear communication between traffic services on the ground and flightcrews. 
This page intentionally left blank 


\section{Potential Barriers to Implementation of the Recommendations}

Implementing the HF Team's recommendations will be challenging. Many of the recommendations call for changes that will generate resistance. In this section, we identify potential barriers to implementation of the team's recommendations, so that the barriers can be addressed.

We believe the following barriers may exist to varying degrees for all the recommendations:

Resources. Considering that resources (i.e., people, money, and time) are always in short supply, there must be a very compelling reason to invest resources in a particular activity.

Liability. Incorporating improvements that can be linked to a safety issue may lead to legal concerns over liability issues, reducing the incentives for making the improvements.

Resistance to change. There is a natural tendency to resist many types of changes, especially if individuals, groups, or organizations feel threatened. For each of these recommendations, it will be important to communicate the intent and potential safety benefits.

Turf protection. This is another common response to change, especially change that is potentially major.

Defensiveness. Another common response is defensiveness against perceived criticism.

Finger pointing. Problems are typically identified as the other party's fault, particularly when system-related deficiencies are involved. We observed several cases of finger pointing by different groups who provided input to this study.

Perception of too much FAA participation and scrutiny. We heard many concerns about increased and inappropriate regulatory oversight.

Misunderstandings about Human Factors. There are many misunderstandings about human factors, what it means, and what it involves. A few that we encountered are:

- There is a single, agreed-upon definition of human factors. We found that it was difficult to find a commonly agreed-upon definition. For example, we found that some people use "human factors" and "crew resource management (CRM)" to mean the same thing. Human factors is much broader than CRM, although CRM is certainly an important part of human factors. 
- There are no special skills or training required to do human factors work (corollary: We're all human, so we can all do human factors). There is a common misperception that anyone can "do human factors" (whatever is meant by that -- see previous bullet) because they are human. Therefore, for example, they can design or evaluate a display or interface based on personal preferences. This perception of human factors overlooks the vast amount of objective, systematic methods and data developed from theoretical and empirical human factors efforts done for a variety of applications. Knowledge of these methods and data is important for appropriately applying human factors. A related and important point that is often overlooked is that subjective opinion or judgment may differ significantly from objective performance results. Judgments and opinions can be very valuable for gaining insight, but are not satisfactory substitutes for objective performance data.

- Experienced pilots are the same as human factors experts. As mentioned in the last point, human factors work requires special knowledge and skills. Piloting skills are equally valuable but are different from human factors skills.

- Test pilots and line pilots can do equivalent functions. Line pilots are not trained in the skills necessary to assess the acceptability of systems across the range of potential failure scenarios, but they can provide valuable insight into how the systems will be used in the operational environment. On the other hand, test pilots are specially trained in evaluation skills, but may lack the operational experience to consider the full range of behaviors that are exhibited by different line pilots.

- It's easy to know when the pilot's information or mission requirements are met -- we know it when we see it. This misperception is similar to the assumption that subjective opinion or judgment is sufficient to determine that requirements are met. As said before, while useful, subjective data are insufficient. In addition to subjective judgment and opinion, the HF Team advocates using objective, systematically acquired data for making design and evaluation decisions.

- Human factors professionals can "human factor" the interface after the design is finished. As discussed earlier in the report, designing a system to be human centered requires consideration of human performance in defining the functionality of the system. Simply putting a well-designed interface on the system after its functionality is already defined is insufficient. For example, improving flight mode annunciation alone without reconsidering the definitions of the autoflight modes will not solve the mode awareness vulnerabilities.

- There is a simple, single-point solution to every human factors problem. As mentioned earlier, the issues we identified are highly interrelated. It is unrealistic to assume that simple, single-point solutions will usually solve human factors problems.

\footnotetext{
${ }^{27}$ What Avionics Engineers Need to Know About Human Factors. Victor Riley, 1995 Digital Avionics Systems
} Conference. 
- We don't need to fix the design - just train the pilots more. Training should not be used as the solution for inadequate design, although sometimes the only short-term approach to dealing with design problems is through training. However, longer-term solutions for improved design should also be pursued.

- Current experience is always applicable to new technology. While sometimes true, it is risky to assume that new technology will have the same influence on human performance as current experience with current technology.

- HF evaluation is a democratic process. Just because more than half of a number of evaluators (or test subjects) have a certain opinion or judgment does not necessarily make that judgment the "right" answer from a human performance perspective.

- Existence of a HF department means HF is a part of design process. The existence of a human factors department does not ensure that human factors have been adequately considered as a core discipline in design (or in other relevant areas, such as training or certification). 
This page intentionally left blank 


\title{
Follow-On Effort and Implementation of the Recommendations
}

\begin{abstract}
Although a great deal of effort has gone into the HF Team's activities, the really difficult tasks lie ahead. The recommendations contained within this report potentially affect many areas within the FAA and industry, including aircraft design and certification, flight operations, flightcrew training, air traffic control, and research and development. Implementation of these recommendations will require a commitment by the affected organizations and individuals. Without commitment, this effort could be soon forgotten, the report put on a shelf, and the status quo maintained.

Therefore, rather than being viewed as the end of a project, this report represents the beginning of what must be a long term commitment by the FAA and the aviation community that will, if successfully implemented and managed, help reduce the accident rate and improve the safety of air transportation. As the first phase of this effort comes to a close, and to assure necessary improvements in the safety of air transportation, it is important that the FAA commit the necessary resources to implementing the recommendations presented in this report.
\end{abstract}

\section{What's Next?}

Since so many FAA organizations are affected by the recommendations, a means of tracking and guiding the implementation of the recommendations will be necessary. Therefore, the HF Team recommends that an implementation team be formed. This team would be tasked with coordinating the implementation of the recommendations and providing visibility to FAA management on the progress of the implementation effort. Such a team could assist affected FAA organizations by interpreting the recommendations to ensure a clear understanding of what the Team intended, identify short and long term priorities, help schedule implementation efforts, and help identify and obtain necessary resources. The implementation team should have members representing each of the FAA organizations responsible for implementing recommendations and should include representatives from the HF Team. The JAA should also be represented. The implementation team should also work with outside industry groups, such as AIA, ATA, ALPA, APA, and SAE G-10, who will also be working to implement this report's recommendations. Figure 7 shows a graphical representation of the proposed FAA implementation team's inter-relationship with affected organizations within and outside the FAA.

One of the first tasks of the implementation team should be to identify and work with the affected organizations and develop an implementation plan, including priorities, specific approaches to implement particular recommendations, and a schedule. The schedule would be based on the urgency and importance of accomplishing the recommendation(s) and the available resources. 


\section{International Effort}

Many of the issues and recommendations contained within this report address areas that must be viewed in international terms. Therefore, implementation of these recommendations should be a coordinated effort with the JAA and other airworthiness authorities as appropriate (e.g., Japan Civil Aviation Bureau, Australian Civil Aviation Authority).

The JAA formed a human factors steering group (HFStG) to consider all human factors aspects of the JARs. One of the tasks of the steering group is to: "...collect information on Human Factors issues and participate, when required, into the activities of other regulatory bodies or organizations (notably FAA)." The JAA HFStG will form a working group to consider the recommendations contained in this report.

The timing with respect to the formation of the JAA HFStG and the release of this report creates an opportunity for coordinating the Human Factors Team's recommendations with the JAA. The FAA has been invited to participate in the JAA HFStG as an observer and has accepted that invitation by naming two individuals to represent the FAA. 
Figure 7

\section{Proposal for FAA Human Factors Team Follow-on Effort}

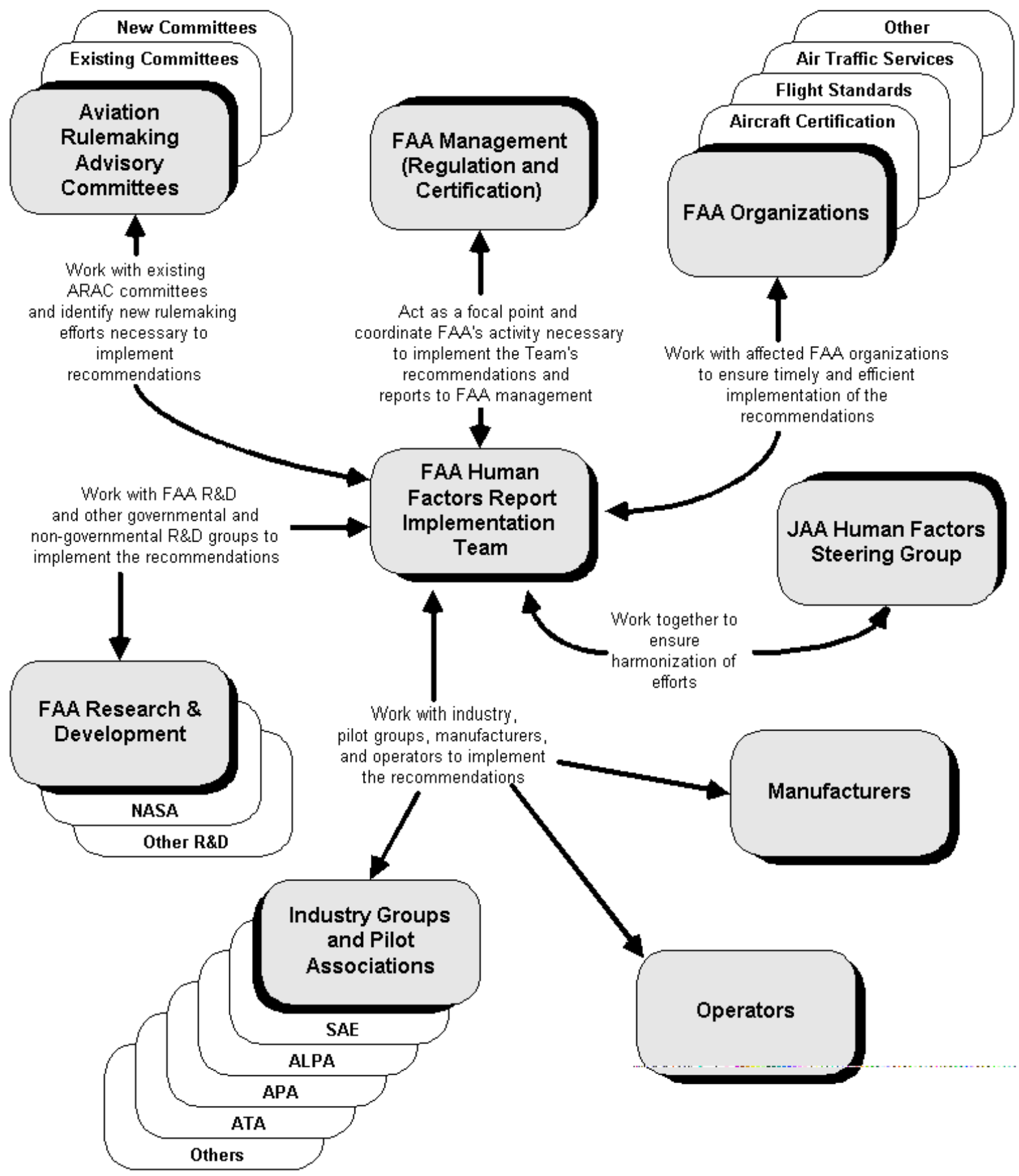


This page intentionally left blank 


\section{Concluding Remarks}

In summary, the HF Team found during its investigation that the aviation system is very safe, but that vulnerabilities in the flightcrew/automation interface exist, especially in the area of flightcrew management of automation and situation awareness. These vulnerabilities appear to exist to varying degrees across the current fleet of transport category airplanes in our study, regardless of the manufacturer, the operator, or whether accidents have occurred in a particular airplane type. Although the Team found specific issues associated with particular design, operating, or training philosophies, we consider the generic vulnerabilities and issues the more significant barrier to improving safety. It is this larger pattern that serves as a barrier to needed improvements to the current level of safety, or could threaten the current safety record in the future aviation environment. It is this larger pattern that needs to be characterized, understood, and addressed.

The issues identified by the HF Team are highly interrelated, and are evidence of aviation system problems, not just isolated human or machine errors. Therefore, we need system solutions, not just point solutions to individual problems. To treat one issue (or underlying cause) in isolation may improve certain aspects of the aviation system, but will ultimately fail to fundamentally increase the safety of airplane operations, and will prevent us from reaching the goal of zero accidents.

The recommendations represent both short term and long term approaches to addressing the issues. These recommendations address the immediate vulnerabilities, as well as the characteristics of the processes in the aviation system that allow the vulnerabilities to exist.

The HF Team chose not to prioritize the recommendations. We decided that the prioritization task was best left to the implementation team proposed in the previous section. Also, we were concerned that prioritization would result in implementation of only those recommendations designated as high priority. While we do believe that some recommendations may be more urgent than others, we believe that all the recommendations are important. As stated in the preceding paragraph, the HF Team believes a systematic approach must be taken to treating the issues if the goal of zero accidents is to be obtained.

The HF team also recognizes the economic pressures that inhibit making safety changes when there is not a strong tie to an accident (and sometimes not even then). However, we believe that, if action is not taken soon, the vulnerabilities we identified have the potential to lead to more accidents and serious incidents.

Commitment by the affected organizations will be necessary if the recommendations in this report are to make a difference in aviation safety. We believe that the FAA has demonstrated its commitment by conducting this study. The next step will require the FAA and the rest of the aviation community to develop innovative approaches to further improve the safety of air transportation. Similarly, airplane and avionics manufacturers, operators, unions, and 
associations must also be willing to work with the FAA and JAA to implement the recommendations. 
Appendices 
This page intentionally left blank 


\section{Appendix A}

\section{Charter Statement}

Human Factor Team to Study Interfaces between Flightcrews and Modern Flight Deck Systems

March 1, 1995 


\section{Background:}

Pilot error continues to dominate the list of primary cause factors for accidents involving transport category airplanes. In approximately $60 \%$ of the accidents with known causes, pilot error was identified as a primary cause factor. Modern flight deck designs, which have automated many pilot tasks, have not significantly reduced this percentage. Although the automatic systems have reduced or eliminated some types of pilot errors, other types of errors have been introduced. Several recent accidents appear to highlight difficulties in pilot interaction with the increasing flight deck automation.

Therefore, the Federal Aviation Administration Transport Airplane Directorate, under the approval of the Director, Aircraft Certification Service, has initiated a study to evaluate current generation transport category airplane flight deck design, especially in regards to the human interfaces with airplane systems and the effect of these interfaces on airplane safety. The study will concentrate on the design, training/crew qualification, and operation of those systems dealing with flight path management. ${ }^{28}$ It will encompass large transport category airplanes equipped with current generation flight deck controls, e.g., Airbus A300-600/ A310/A320/ A330/A340, Boeing 737/757/767/747-400/777, McDonnell Douglas MD80/MD-90/MD-11 and Fokker F28-0100 and -0070.

To conduct this study, a team, entitled the Human Factors Team (hereafter is referred to as the Team), has been formed.

\section{Statement of Objectives:}

The Team will evaluate current generation transport category airplane flight deck designs in regards to the human interfaces with airplane systems and the effect of these interfaces on airplane safety. The study will concentrate on the design, training/crew qualification, and operation of those systems dealing with flight path management. The Team will consider all factors that can influence the pilot's ability to safely operate the airplane during all phases of flight, including, but not limited to, mode/situation awareness, pilot expectations regarding the automatic systems and the subsequent pilot response when those expectations are not met, and crew resource management in modern flight decks.

The Team shall:

a) Identify specific and generic safety related design problems, if any, related to pilot/airplane interfaces, in the airplane types under study. The Team will recommend appropriate means to address these problems.

\footnotetext{
${ }^{28}$ Flight path management is defined as the integration of guidance, navigation, control and associated interfaces/control devices used by the pilot to manage the flight path of the aircraft.
} 
b) Identify specific and generic training/crew qualification and operational problems, if any, related to pilot/airplane interfaces in the airplane types under study. The Team will recommend appropriate means to address these problems.

c) Identify those concerns that should be the subject of new or revised Federal Aviation Regulations (FAR), advisory circulars (AC) and/or policies.

\section{Principal Types to be Studied:}

The following series airplanes are to be addressed by the Team:

Boeing: Models 737/757/767/747-400/777

Airbus: Models A300-600/A310/A320/A330/A340

McDonnell Douglas: Models MD-80/MD-90/MD-11

Fokker: Model F28-0100/-0070

The Team will visit each manufacturer of the airplane types under study.

\section{Time Frame:}

The study shall be completed within 12 months following the first team meeting.

\section{Group Structure:}

The Team will consist of approximately 11 individuals and is composed as follows: (1) two engineers from the FAA Transport Standards Staff; (2) two FAA flight test pilots; (3) the FAA National Resource Specialists in flight management and air carrier operations; (4) one pilot from FAA Flight Standards; (5) one NASA human factors expert; and (6) three representatives from the Joint Aviation Authorities (JAA). The Team will have co-leaders, two from the FAA and one from outside the FAA. In addition, three independent consultants with recognized expertise in human factors will be retained as expert technical advisors to the team. Other experts in the areas of study will be consulted as necessary.

\section{Group Membership:}

Team membership is as follows:

FAA Co-chairs: $\quad$ Steve Slotte and Don Stimson, Transport Standards Staff NASA Co-Chair: $\quad$ Dr. Kathy Abbott, NASA 
JAA Participants: Dr. René Amalberti (DGAC - France) - Human Factors Specialist François Fabre (DGAC - France) - Test Pilot

Terry Newman (CAA - U.K.) - Test Pilot

Heert Tigchelaar (RLD - Netherlands) - Test Pilot

FAA Participants: Eugene Bollin, Wichita Aircraft Certification Office Flight Test

Tom Imrich, National Resource Specialist -- Air Carrier Operations

Rod Lalley, Seattle Aircraft Evaluation Group

George Lyddane, National Resource Specialist -- Flight Management

Guy Thiel, Los Angeles Aircraft Certification Office Flight Test

\section{Expert Technical Advisors:}

Independent

Consultants: $\quad$ Dr. Bob Helmreich, University of Texas

Dr. Nadine Sarter, Ohio State University

Dr. David Woods, Ohio State University

\section{Product:}

Interim: A brief report on the Team's progress and plans will be submitted at bi-monthly intervals to the Manager, Transport Airplane Directorate.

Final: The Team will prepare a final report that defines the Team's findings and recommendations. The final report will be submitted to the Manager, Transport Airplane Directorate.
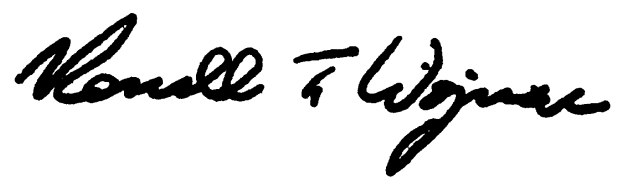

March 1, 1995

Ronald T. Wojnar

Manager, Transport Airplane Directorate 


\section{Appendix B \\ Matrix of Issues and Recommendations}

\begin{tabular}{|c|c|c|}
\hline Issue & Recommendation & $\begin{array}{l}\text { Recommendation } \\
\text { Number }\end{array}$ \\
\hline $\begin{array}{l}\text { Measurement of and Incentives } \\
\text { for Safety }\end{array}$ & $\begin{array}{l}\text { The FAA should: } \\
\text { - } \quad \text { Lead the aviation community } \\
\text { to use accident precursors } \\
\text { increasingly and consistently as } \\
\text { an additional measure of } \\
\text { aviation safety; } \\
\text { - Work with industry to establish } \\
\text { systems/processes for } \\
\text { collecting precursor data and } \\
\text { for tracking the influence of } \\
\text { system changes (e.g., design } \\
\text { changes, training changes) on } \\
\text { safety; and } \\
\text { Work with industry to } \\
\text { investigate other means of } \\
\text { assessing or communicating } \\
\text { safety (e.g., ways of measuring } \\
\text { errors intercepted, incidents or } \\
\text { accidents prevented, etc.). }\end{array}$ & Measures-1 \\
\hline
\end{tabular}




\begin{tabular}{|c|c|c|}
\hline Issue & Recommendation & $\begin{array}{c}\text { Recommendation } \\
\text { Number }\end{array}$ \\
\hline $\begin{array}{l}\text { Measurement of and Incentives } \\
\text { for Safety }\end{array}$ & $\begin{array}{l}\text { In accident/incident investigations } \\
\text { where human error is considered a } \\
\text { potential factor, the FAA and the } \\
\text { National Transportation Safety } \\
\text { Board should thoroughly } \\
\text { investigate the factors that } \\
\text { contributed to the error, including } \\
\text { design, training, operational } \\
\text { procedures, the airspace system, or } \\
\text { other factors. The FAA should } \\
\text { encourage other organizations } \\
\text { (both domestic and foreign) } \\
\text { conducting accident/incident } \\
\text { investigations to do the same. This } \\
\text { recommendation should apply to } \\
\text { all accident/incident investigations } \\
\text { involving human error, regardless } \\
\text { of whether the error is associated } \\
\text { with a pilot, mechanic, air traffic } \\
\text { controller, dispatcher, or other } \\
\text { participant in the aviation system. }\end{array}$ & Measures-2 \\
\hline $\begin{array}{l}\text { Measurement of and Incentives } \\
\text { for Safety }\end{array}$ & $\begin{array}{l}\text { The FAA should explore means to } \\
\text { create additional incentives to } \\
\text { improve safety through } \\
\text { appropriate design, training or } \\
\text { operational improvements. }\end{array}$ & Measures-3 \\
\hline $\begin{array}{l}\text { Flightcrew Management and } \\
\text { Direction of Automation }\end{array}$ & $\begin{array}{l}\text { The FAA should ensure that a } \\
\text { uniform set of information } \\
\text { regarding the manufacturers' and } \\
\text { operators' automation philosophies } \\
\text { is explicitly conveyed to } \\
\text { flightcrews. }\end{array}$ & AutomationMgt-1 \\
\hline
\end{tabular}




\begin{tabular}{|c|c|c|}
\hline Issue & Recommendation & $\begin{array}{c}\text { Recommendation } \\
\text { Number }\end{array}$ \\
\hline $\begin{array}{l}\text { Flightcrew Management and } \\
\text { Direction of Automation }\end{array}$ & $\begin{array}{l}\text { The FAA should require } \\
\text { operators' manuals and } \\
\text { initial/recurrent qualification } \\
\text { programs to provide clear and } \\
\text { concise guidance on: } \\
\text { - Examples of circumstances in } \\
\text { which the autopilot should be } \\
\text { engaged, disengaged, or used } \\
\text { in a mode with greater or } \\
\text { lesser authority; } \\
\text { The conditions under which the } \\
\text { autopilot or autothrottle will or } \\
\text { will not engage, will disengage, } \\
\text { or will revert to another mode; } \\
\text { and } \\
\text { Appropriate combinations of } \\
\text { automatic and manual flight } \\
\text { path control (e.g., autothrottle } \\
\text { engaged with the autopilot } \\
\text { off). }\end{array}$ & AutomationMgt-2 \\
\hline
\end{tabular}




\begin{tabular}{|c|c|c|}
\hline Issue & Recommendation & $\begin{array}{c}\text { Recommendation } \\
\text { Number }\end{array}$ \\
\hline $\begin{array}{l}\text { Flightcrew Management and } \\
\text { Direction of Automation }\end{array}$ & $\begin{array}{l}\text { The FAA should initiate a review } \\
\text { of the autopilots on all transport } \\
\text { category airplanes to identify the } \\
\text { potential for producing hazardous } \\
\text { energy states, excessive pitch or } \\
\text { bank angles, subtle departures } \\
\text { from the intended flight path, } \\
\text { slow-overs, hard-overs, or other } \\
\text { undesirable maneuvers. Results of } \\
\text { this review should be the basis for } \\
\text { initiating appropriate actions, such } \\
\text { as design improvements, flight } \\
\text { manual revisions, additional } \\
\text { operating limitations, or changes in } \\
\text { training programs or operational } \\
\text { procedures. }\end{array}$ & AutomationMgt-3 \\
\hline $\begin{array}{l}\text { Flightcrew Management and } \\
\text { Direction of Automation }\end{array}$ & $\begin{array}{l}\text { The FAA should assure that } \\
\text { analyses are conducted to better } \\
\text { understand why flightcrews deviate } \\
\text { from procedures, especially when } \\
\text { the procedural deviation } \\
\text { contributes to causing or } \\
\text { preventing an accident or incident. }\end{array}$ & AutomationMgt-4 \\
\hline $\begin{array}{l}\text { Flightcrew Management and } \\
\text { Direction of Automation }\end{array}$ & $\begin{array}{l}\text { The FAA should request industry } \\
\text { to take the lead in developing } \\
\text { design guidelines for the next } \\
\text { generation of flight management } \\
\text { systems }\end{array}$ & AutomationMgt-5 \\
\hline
\end{tabular}




\begin{tabular}{|c|c|c|}
\hline Issue & Recommendation & $\begin{array}{c}\text { Recommendation } \\
\text { Number }\end{array}$ \\
\hline Flightcrew Situation Awareness & $\begin{array}{l}\text { The FAA should require operators } \\
\text { to increase flightcrews' } \\
\text { understanding of and sensitivity to } \\
\text { maintaining situation awareness, } \\
\text { particularly: } \\
\text { - Mode and airplane energy } \\
\text { awareness issues associated } \\
\text { with autoflight systems (i.e., } \\
\text { autopilot, autothrottle, flight } \\
\text { management system, and fly- } \\
\text { by-wire flight control systems); } \\
\text { Position awareness with } \\
\text { respect to the intended flight } \\
\text { path and proximity to terrain, } \\
\text { obstacles, or traffic; and } \\
\text { Potential causes, flightcrew } \\
\text { detection, and recovery from } \\
\text { hazardous pitch or bank angle } \\
\text { upsets while under autopilot } \\
\text { control (e.g., wake vortex, } \\
\text { subtle autopilot failures, engine } \\
\text { failure in cruise, atmospheric } \\
\text { turbulence). }\end{array}$ & SA-1 \\
\hline
\end{tabular}




\begin{tabular}{|c|c|c|}
\hline Issue & Recommendation & $\begin{array}{c}\text { Recommendation } \\
\text { Number }\end{array}$ \\
\hline Flightcrew Situation Awareness & $\begin{array}{l}\text { The FAA should require } \\
\text { operators' initial and recurrent } \\
\text { training programs as well as } \\
\text { appropriate operating manuals to: } \\
\text { - Explicitly address autoflight } \\
\text { mode and airplane energy } \\
\text { awareness hazards; } \\
\text { - Provide information on the } \\
\text { characteristics and principles of } \\
\text { the autoflight system's design } \\
\text { that have operational safety } \\
\text { consequences; and } \\
\text { Provide training to proficiency } \\
\text { of the flight management } \\
\text { system capabilities to be used } \\
\text { in operations. }\end{array}$ & SA-2 \\
\hline Flightcrew Situation Awareness & $\begin{array}{l}\text { The FAA should encourage the } \\
\text { aviation industry to develop and } \\
\text { implement new concepts to } \\
\text { provide better terrain awareness. }\end{array}$ & $\mathrm{SA}-3$ \\
\hline Flightcrew Situation Awareness & $\begin{array}{l}\text { The FAA and the aviation industry } \\
\text { should develop and implement a } \\
\text { plan to transition to standardized } \\
\text { instrument approaches using lateral } \\
\text { navigation (LNAV) and vertical } \\
\text { navigation (VNAV) path guidance } \\
\text { for three-dimensional approaches. } \\
\text { The use of approaches that lack } \\
\text { vertical path guidance should be } \\
\text { minimized and eventually } \\
\text { eliminated. }\end{array}$ & $\mathrm{SA}-4$ \\
\hline
\end{tabular}




\begin{tabular}{|c|c|c|}
\hline Issue & Recommendation & $\begin{array}{c}\text { Recommendation } \\
\text { Number }\end{array}$ \\
\hline Flightcrew Situation Awareness & $\begin{array}{l}\text { The FAA should encourage the } \\
\text { exploration, development, and } \\
\text { testing of new ideas and } \\
\text { approaches for providing effective } \\
\text { feedback to the flightcrew to } \\
\text { support error detection and } \\
\text { improved situation awareness. }\end{array}$ & SA-5 \\
\hline Flightcrew Situation Awareness & $\begin{array}{l}\text { The FAA should encourage } \\
\text { standardization, as appropriate, of } \\
\text { automation interface features, such } \\
\text { as: } \\
\text { - The location, shape, and } \\
\text { direction of movement for } \\
\text { takeoff/go-around and } \\
\text { autothrottle quick disconnect } \\
\text { switches; } \\
\text { - Autoflight system mode } \\
\text { selectors and selector panel } \\
\text { layout; } \\
\text { - Autoflight system modes, } \\
\text { display symbology, and } \\
\text { nomenclature; and } \\
\text { Flight management system } \\
\text { interfaces, data entry } \\
\text { conventions, and } \\
\text { nomenclature. }\end{array}$ & SA-6 \\
\hline
\end{tabular}




\begin{tabular}{|l|l|c|}
\hline Issue & \multicolumn{1}{|c|}{ Recommendation } & $\begin{array}{c}\text { Recommendation } \\
\text { Number }\end{array}$ \\
\hline Flightcrew Situation Awareness & $\begin{array}{l}\text { The FAA and the aviation industry } \\
\text { should update or develop new } \\
\text { standards and evaluation criteria } \\
\text { for information presented to the } \\
\text { flightcrew by flight deck displays } \\
\text { and audio advisories (e.g., primary } \\
\text { flight displays, } \\
\text { navigation/communication } \\
\text { displays, synoptics showing system } \\
\text { states). }\end{array}$ & SA-7 \\
\hline Flightcrew Situation Awareness & $\begin{array}{l}\text { The FAA should ensure that } \\
\text { flightcrews are educated about } \\
\text { hazardous states of awareness and } \\
\text { the need for countermeasures to } \\
\text { maintain vigilance. The FAA } \\
\text { should encourage operators to: } \\
\text { Develop operational } \\
\text { procedures and strategies to } \\
\text { foster attention management } \\
\text { skills with the objective of } \\
\text { avoiding hazardous states of } \\
\text { awareness; and } \\
\text { Develop techniques to apply } \\
\text { during training to identify and } \\
\text { minimize hazardous states of } \\
\text { awareness. }\end{array}$ & SA-8 \\
\hline
\end{tabular}




\begin{tabular}{|c|c|c|}
\hline Issue & Recommendation & $\begin{array}{c}\text { Recommendation } \\
\text { Number }\end{array}$ \\
\hline Flightcrew Situation Awareness & $\begin{array}{l}\text { The FAA should sponsor research, } \\
\text { or assure that research is } \\
\text { accomplished, to develop } \\
\text { improved methods for: } \\
\text { - Evaluating designs for } \\
\text { susceptibility to hazardous } \\
\text { states of awareness (e.g., } \\
\text { underload, complacency, } \\
\text { absorption); and } \\
\text { Training to minimize } \\
\text { hazardous states of awareness. }\end{array}$ & SA-9 \\
\hline $\begin{array}{l}\text { Communication and } \\
\text { Coordination }\end{array}$ & $\begin{array}{l}\text { The FAA should identify existing } \\
\text { air traffic procedures that are } \\
\text { incompatible with highly } \\
\text { automated airplanes. These } \\
\text { incompatible procedures should be } \\
\text { discontinued or modified as soon } \\
\text { as feasible. }\end{array}$ & Comm/Coord-1 \\
\hline $\begin{array}{l}\text { Communication and } \\
\text { Coordination }\end{array}$ & $\begin{array}{l}\text { The FAA should task an existing } \\
\text { advisory group or, if necessary, } \\
\text { establish a new forum to ensure } \\
\text { coordination between the design of } \\
\text { air traffic procedures and the } \\
\text { design and operation of highly } \\
\text { automated airplanes. }\end{array}$ & Comm/Coord-2 \\
\hline
\end{tabular}




\begin{tabular}{|c|c|c|}
\hline Issue & Recommendation & $\begin{array}{c}\text { Recommendation } \\
\text { Number }\end{array}$ \\
\hline $\begin{array}{l}\text { Communication and } \\
\text { Coordination }\end{array}$ & $\begin{array}{l}\text { The FAA should lead an industry- } \\
\text { wide effort to share safety } \\
\text { information obtained from in- } \\
\text { service data and from difficulties } \\
\text { encountered in training. This effort } \\
\text { should be capable of assisting in } \\
\text { the identification and resolution of } \\
\text { problems attributed to flightcrew } \\
\text { error. }\end{array}$ & Comm/Coord-3 \\
\hline $\begin{array}{c}\text { Communication and } \\
\text { Coordination }\end{array}$ & $\begin{array}{l}\text { The FAA should require operators } \\
\text { to have an appropriate process, } \\
\text { with demonstrated effectiveness, } \\
\text { for informing flightcrews about } \\
\text { relevant accidents, incidents, in- } \\
\text { service problems, and problems } \\
\text { encountered in training that could } \\
\text { affect flight safety. }\end{array}$ & Comm/Coord-4 \\
\hline $\begin{array}{c}\text { Communication and } \\
\text { Coordination }\end{array}$ & $\begin{array}{l}\text { The FAA should encourage the } \\
\text { redesign and modernization of the } \\
\text { information provided to the } \\
\text { flightcrew in notices to airmen } \\
\text { (NOTAMs), charts, approach } \\
\text { plates, instrument procedures, } \\
\text { meteorological data, etc. The } \\
\text { information should be prioritized } \\
\text { and highlighted in terms of urgency } \\
\text { and importance, and presented in a } \\
\text { clear, well-organized, easy-to- } \\
\text { understand format suitable for use } \\
\text { with current and future airplanes. }\end{array}$ & Comm/Coord-5 \\
\hline
\end{tabular}




\begin{tabular}{|c|c|c|}
\hline Issue & Recommendation & $\begin{array}{c}\text { Recommendation } \\
\text { Number }\end{array}$ \\
\hline $\begin{array}{l}\text { Communication and } \\
\text { Coordination }\end{array}$ & $\begin{array}{l}\text { The FAA should improve and } \\
\text { increase interaction between the } \\
\text { Flight Standards and Aircraft } \\
\text { Certification Services. }\end{array}$ & Comm/Coord-6 \\
\hline $\begin{array}{l}\text { Communication and } \\
\text { Coordination }\end{array}$ & $\begin{array}{l}\text { The FAA and industry should } \\
\text { improve the coordination and } \\
\text { distribution of tasks undertaken by } \\
\text { federal advisory committees and } \\
\text { industry technical committees to } \\
\text { reduce overlap and avoid } \\
\text { duplication of effort. }\end{array}$ & Comm/Coord-7 \\
\hline $\begin{array}{l}\text { Communication and } \\
\text { Coordination }\end{array}$ & $\begin{array}{l}\text { The FAA should improve } \\
\text { communication about research } \\
\text { programs, research results, and } \\
\text { advances in technology to } \\
\text { appropriate FAA personnel. }\end{array}$ & Comm/Coord-8 \\
\hline $\begin{array}{l}\text { Communication and } \\
\text { Coordination }\end{array}$ & $\begin{array}{l}\text { The FAA should hold research } \\
\text { funding sponsors and researchers } \\
\text { accountable for supporting the } \\
\text { transfer of research results. }\end{array}$ & Comm/Coord-9 \\
\hline $\begin{array}{l}\text { Communication and } \\
\text { Coordination }\end{array}$ & $\begin{array}{l}\text { The FAA should assure strategic } \\
\text { leadership and support } \\
\text { establishment of a coordinated } \\
\text { research portfolio in aviation } \\
\text { human factors on the national and } \\
\text { international levels. }\end{array}$ & Comm/Coord-10 \\
\hline
\end{tabular}




\begin{tabular}{|c|c|c|}
\hline Issue & Recommendation & $\begin{array}{c}\text { Recommendation } \\
\text { Number }\end{array}$ \\
\hline $\begin{array}{c}\text { Processes for Design, } \\
\text { Regulatory, and Training } \\
\text { Activities }\end{array}$ & $\begin{array}{l}\text { The FAA should task an aviation } \\
\text { industry working group to produce } \\
\text { a set of guiding principles for } \\
\text { designers to use as a recommended } \\
\text { practice in designing and } \\
\text { integrating human-centered flight } \\
\text { deck automation. }\end{array}$ & Processes-1 \\
\hline $\begin{array}{c}\text { Processes for Design, } \\
\text { Regulatory, and Training } \\
\text { Activities }\end{array}$ & $\begin{array}{l}\text { The FAA should establish } \\
\text { regulatory and associated advisory } \\
\text { material to require the use of a } \\
\text { flight deck certification review } \\
\text { process that addresses human } \\
\text { performance considerations. }\end{array}$ & Processes-2 \\
\hline $\begin{array}{c}\text { Processes for Design, } \\
\text { Regulatory, and Training } \\
\text { Activities }\end{array}$ & $\begin{array}{l}\text { The FAA and the aviation industry } \\
\text { should investigate the use of } \\
\text { innovative training tools and } \\
\text { methods to expand pertinent safety } \\
\text { related knowledge of flightcrews } \\
\text { on a continuing basis. The FAA } \\
\text { and the aviation industry should } \\
\text { explore incentives to encourage } \\
\text { continued training and education } \\
\text { beyond the minimum required by } \\
\text { the current regulations. }\end{array}$ & Processes-3 \\
\hline $\begin{array}{l}\text { Criteria, Regulatory Standards, } \\
\text { Methods and Tools for Design } \\
\text { and Certification }\end{array}$ & $\begin{array}{l}\text { The FAA should require evaluation } \\
\text { of flight deck designs for } \\
\text { susceptibility to design-induced } \\
\text { flightcrew errors and the } \\
\text { consequences of those errors as } \\
\text { part of the type certification } \\
\text { process. }\end{array}$ & Criteria-1 \\
\hline
\end{tabular}




\begin{tabular}{|c|l|c|}
\hline Issue & \multicolumn{1}{|c|}{ Recommendation } & $\begin{array}{c}\text { Recommendation } \\
\text { Number }\end{array}$ \\
\hline $\begin{array}{c}\text { Criteria, Regulatory Standards, } \\
\text { Methods and Tools for Design } \\
\text { and Certification }\end{array}$ & $\begin{array}{l}\text { The FAA should prepare and } \\
\text { distribute interim guidance material } \\
\text { that updates current autopilot } \\
\text { certification policy. }\end{array}$ & Criteria-3 \\
\hline $\begin{array}{c}\text { Criteria, Regulatory Standards, } \\
\text { and Certification }\end{array}$ & $\begin{array}{l}\text { The FAA should task an } \\
\text { appropriate Aviation Rulemaking } \\
\text { Advisory Committee } \\
\text { Harmonization Working Group } \\
\text { (HWG) with updating the } \\
\text { autopilot regulatory standards } \\
\text { (14 CFR 25.1329). This HWG } \\
\text { should include specialists } \\
\text { knowledgeable in human factors } \\
\text { methods and skills from both } \\
\text { industry and the regulatory } \\
\text { authorities. }\end{array}$ \\
\hline
\end{tabular}




\begin{tabular}{|c|l|l|}
\hline \multicolumn{1}{|c|}{ Issue } & \multicolumn{1}{|c|}{ Recommendation } & $\begin{array}{c}\text { Recommendation } \\
\text { Number }\end{array}$ \\
\hline $\begin{array}{c}\text { Methods and Tools for Design } \\
\text { and Certification }\end{array}$ & $\begin{array}{l}\text { The FAA should revise/update the } \\
\text { following specific FARs and } \\
\text { associated advisory material: } \\
\S 25.1322 \text { Warning, caution, and } \\
\text { advisory lights: Revise to reflect } \\
\text { the current and anticipated design } \\
\text { practice for modern transport } \\
\text { category airplanes. } \\
\S 25.1335 \text { Flight Director: Revise } \\
\text { to reflect the current and } \\
\text { anticipated design practice for } \\
\text { modern transport category } \\
\text { airplanes. } \\
\S \text { 121.703 Mechanical reliability } \\
\text { reports: Revise the requirements to } \\
\text { also include reporting of significant } \\
\text { flight deck automation failures } \\
\text { and/or anomalies that adversely } \\
\text { affect safe flight path management. } \\
\text { Reinforce the Aviation Rulemaking } \\
\text { Advisory Committee (ARAC) } \\
\text { activity in this area. }\end{array}$ & \multicolumn{1}{|c}{} \\
\hline
\end{tabular}




\begin{tabular}{|c|c|c|}
\hline Issue & Recommendation & $\begin{array}{c}\text { Recommendation } \\
\text { Number }\end{array}$ \\
\hline $\begin{array}{l}\text { Knowledge and Skills of } \\
\text { Designers, Pilots, Operators, } \\
\text { Regulators, and Researchers }\end{array}$ & $\begin{array}{l}\text { The FAA should encourage flight } \\
\text { deck design organizations to: } \\
\text { (1) Make human factors } \\
\text { engineering a core discipline } \\
\text { of the flight deck system } \\
\text { design activity; and } \\
\text { (2) Ensure that the design team has } \\
\text { sufficient human factors and } \\
\text { operational knowledge and } \\
\text { expertise by: } \\
\text { Distributing guiding principles } \\
\text { for flightcrew-centered design } \\
\text { (as described in } \\
\text { Recommendation Processes-1) } \\
\text { to all design team members; } \\
\text { Including human factors } \\
\text { expertise as part of the design } \\
\text { team; } \\
\text { Assuring that each relevant } \\
\text { member of the team has at } \\
\text { least a basic knowledge of } \\
\text { human factors in order to } \\
\text { understand and communicate } \\
\text { human performance issues and } \\
\text { human-centered design } \\
\text { considerations; and } \\
\text { Assuring that flight deck } \\
\text { design team members have } \\
\text { relevant operational } \\
\text { knowledge. }\end{array}$ & Knowledge-1 \\
\hline
\end{tabular}




\begin{tabular}{|c|c|c|}
\hline Issue & Recommendation & $\begin{array}{c}\text { Recommendation } \\
\text { Number }\end{array}$ \\
\hline $\begin{array}{l}\text { Knowledge and Skills of } \\
\text { Designers, Pilots, Operators, } \\
\text { Regulators, and Researchers }\end{array}$ & 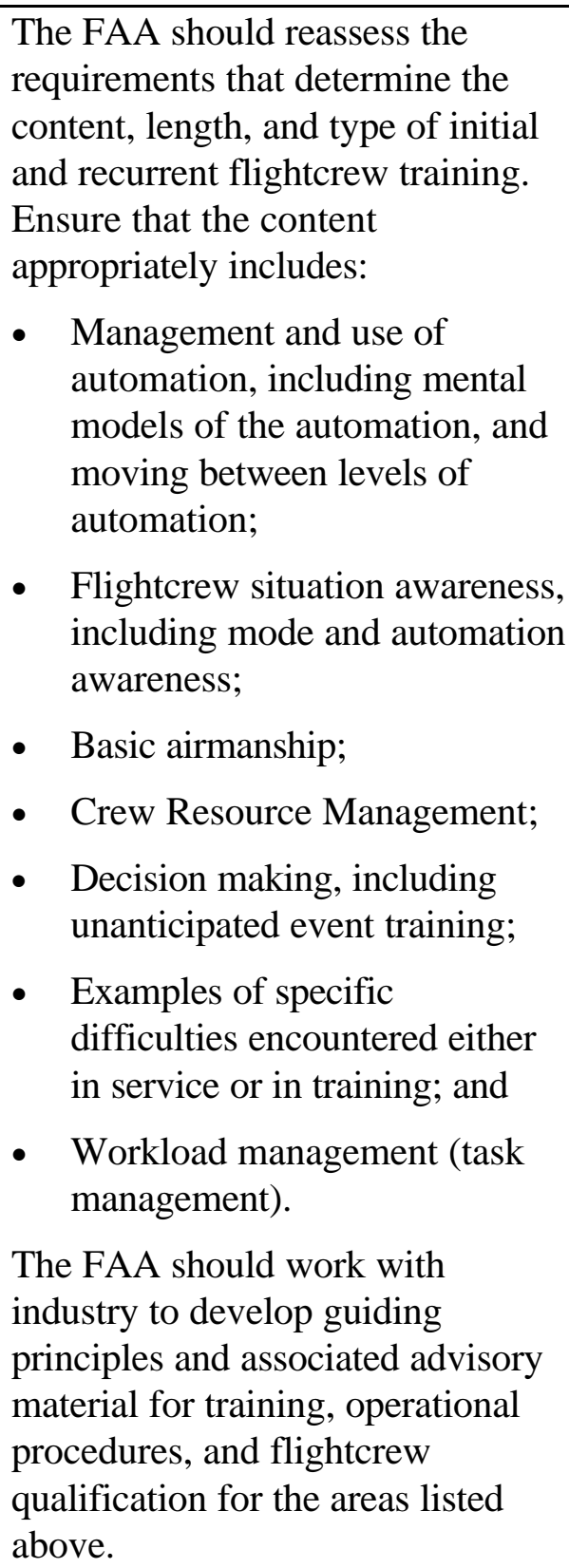 & Knowledge-2 \\
\hline
\end{tabular}




\begin{tabular}{|c|c|c|}
\hline Issue & Recommendation & $\begin{array}{c}\text { Recommendation } \\
\text { Number }\end{array}$ \\
\hline $\begin{array}{l}\text { Knowledge and Skills of } \\
\text { Designers, Pilots, Operators, } \\
\text { Regulators, and Researchers }\end{array}$ & $\begin{array}{l}\text { The FAA should strongly } \\
\text { encourage or provide incentives to } \\
\text { make advanced maneuvers training } \\
\text { an integral part of the training } \\
\text { curriculum, especially in recurrent } \\
\text { training. }\end{array}$ & Knowledge-3 \\
\hline $\begin{array}{l}\text { Knowledge and Skills of } \\
\text { Designers, Pilots, Operators, } \\
\text { Regulators, and Researchers }\end{array}$ & $\begin{array}{l}\text { The FAA should reassess recency } \\
\text { requirements for flightcrews } \\
\text { involved in long haul operations. } \\
\text { Consider providing incentives and } \\
\text { alternative methods for flightcrews } \\
\text { to practice takeoffs and landings, } \\
\text { and perhaps arrival and departure } \\
\text { procedures that are infrequently } \\
\text { used. }\end{array}$ & Knowledge-4 \\
\hline $\begin{array}{l}\text { Knowledge and Skills of } \\
\text { Designers, Pilots, Operators, } \\
\text { Regulators, and Researchers }\end{array}$ & $\begin{array}{l}\text { The FAA should reassess the } \\
\text { airman certification criteria to } \\
\text { ensure that pilots are released with } \\
\text { a satisfactory level of skills for } \\
\text { managing and using automation. } \\
\text { Since current training is often } \\
\text { oriented toward preparing pilots } \\
\text { for checkrides, the airman } \\
\text { certification criteria should be } \\
\text { reassessed to ensure appropriate } \\
\text { coverage of the topics listed in } \\
\text { Recommendation Knowledge- } 2 \text {. }\end{array}$ & Knowledge-5 \\
\hline
\end{tabular}




\begin{tabular}{|c|c|c|}
\hline Issue & Recommendation & $\begin{array}{c}\text { Recommendation } \\
\text { Number }\end{array}$ \\
\hline $\begin{array}{l}\text { Knowledge and Skills of } \\
\text { Designers, Pilots, Operators, } \\
\text { Regulators, and Researchers }\end{array}$ & $\begin{array}{l}\text { Operators should ensure that flight } \\
\text { safety and training managers are } \\
\text { appropriately educated about } \\
\text { human factors considerations, } \\
\text { particularly with regard to } \\
\text { automation. }\end{array}$ & Knowledge-6 \\
\hline $\begin{array}{l}\text { Knowledge and Skills of } \\
\text { Designers, Pilots, Operators, } \\
\text { Regulators, and Researchers }\end{array}$ & $\begin{array}{l}\text { The FAA should improve the } \\
\text { education of Air Traffic Service } \\
\text { personnel about the capabilities } \\
\text { and limitations of highly automated } \\
\text { airplanes. }\end{array}$ & Knowledge-7 \\
\hline $\begin{array}{l}\text { Knowledge and Skills of } \\
\text { Designers, Pilots, Operators, } \\
\text { Regulators, and Researchers }\end{array}$ & $\begin{array}{l}\text { The FAA should provide } \\
\text { appropriate regulatory personnel } \\
\text { with a guide or roadmap to current } \\
\text { Federal Aviation Regulations, } \\
\text { advisory material, policy } \\
\text { memoranda, and other guidance } \\
\text { material dealing with human } \\
\text { performance related to the } \\
\text { flightcrew-vehicle interface. The } \\
\text { FAA should ensure that this } \\
\text { material is used in aircraft } \\
\text { certification projects, airline } \\
\text { qualification program assessments, } \\
\text { and airman qualification. }\end{array}$ & Knowledge- 8 \\
\hline
\end{tabular}




\begin{tabular}{|c|c|c|}
\hline Issue & Recommendation & $\begin{array}{c}\text { Recommendation } \\
\text { Number }\end{array}$ \\
\hline $\begin{array}{l}\text { Knowledge and Skills of } \\
\text { Designers, Pilots, Operators, } \\
\text { Regulators, and Researchers }\end{array}$ & $\begin{array}{l}\text { The FAA should develop a } \\
\text { systematic training program for } \\
\text { appropriate Aircraft Certification } \\
\text { and Flight Standards Services } \\
\text { personnel to provide initial and } \\
\text { recurrent training in the area of } \\
\text { human factors as it relates to } \\
\text { certifying new products and } \\
\text { evaluating flightcrew performance. } \\
\text { The training should include } \\
\text { instruction on: } \\
\text { - Insight into the relationship } \\
\text { among the flightcrew, the flight } \\
\text { deck design, and the } \\
\text { operational environment; } \\
\text { - Flightcrew information } \\
\text { processing; } \\
\text { - Workload, human error, and } \\
\text { situation awareness; } \\
\text { - Other flightcrew performance } \\
\text { issues, including fatigue, CRM, } \\
\text { and attention management; } \\
\text { - Design and evaluation of flight } \\
\text { deck displays; } \\
\text { - Hircraft control laws and } \\
\text { feedback systems; } \\
\text { Human-automation interaction; } \\
\text { to the user. } \\
\text { - }\end{array}$ & Knowledge-9 \\
\hline
\end{tabular}




\begin{tabular}{|c|c|c|}
\hline Issue & Recommendation & $\begin{array}{c}\text { Recommendation } \\
\text { Number }\end{array}$ \\
\hline $\begin{array}{l}\text { Knowledge and Skills of } \\
\text { Designers, Pilots, Operators, } \\
\text { Regulators, and Researchers }\end{array}$ & $\begin{array}{l}\text { The FAA should appropriately } \\
\text { staff the standards organizations } \\
\text { and aircraft certification offices } \\
\text { with human factors expertise and } \\
\text { integrate personnel with such } \\
\text { expertise into certification teams, } \\
\text { participating and applying their } \\
\text { expertise in the same manner as } \\
\text { other certification team members } \\
\text { (e.g. airframe, flight test, systems } \\
\text { and equipment, propulsion). }\end{array}$ & Knowledge-10 \\
\hline $\begin{array}{l}\text { Knowledge and Skills of } \\
\text { Designers, Pilots, Operators, } \\
\text { Regulators, and Researchers }\end{array}$ & $\begin{array}{l}\text { The FAA should increase Aircraft } \\
\text { Certification and Flight Standards } \\
\text { Services personnel's knowledge } \\
\text { about each other's roles and } \\
\text { responsibilities. In particular, } \\
\text { increase certification pilots' and } \\
\text { engineers' knowledge of line } \\
\text { operations considerations, and } \\
\text { Aircraft Evaluation Group } \\
\text { personnel's knowledge about } \\
\text { airworthiness certification } \\
\text { considerations. }\end{array}$ & Knowledge-11 \\
\hline $\begin{array}{l}\text { Knowledge and Skills of } \\
\text { Designers, Pilots, Operators, } \\
\text { Regulators, and Researchers }\end{array}$ & $\begin{array}{l}\text { The FAA should improve the } \\
\text { knowledge of personnel in Aircraft } \\
\text { Certification and Flight Standards } \\
\text { Services about processes for } \\
\text { identifying and communicating } \\
\text { requirements for research (either } \\
\text { specific studies required or } \\
\text { identification of areas of concern). }\end{array}$ & Knowledge-12 \\
\hline
\end{tabular}




\begin{tabular}{|c|c|c|}
\hline Issue & Recommendation & $\begin{array}{c}\text { Recommendation } \\
\text { Number }\end{array}$ \\
\hline $\begin{array}{l}\text { Knowledge and Skills of } \\
\text { Designers, Pilots, Operators, } \\
\text { Regulators, and Researchers }\end{array}$ & $\begin{array}{l}\text { The FAA should encourage } \\
\text { researchers to learn more about } \\
\text { industry and FAA's research needs } \\
\text { and about operational } \\
\text { considerations in aviation. }\end{array}$ & Knowledge-13 \\
\hline $\begin{array}{c}\text { Cultural and Language } \\
\text { Differences }\end{array}$ & $\begin{array}{l}\text { The FAA should ensure that } \\
\text { research is conducted to } \\
\text { characterize cultural effects and } \\
\text { provide better methods to adapt } \\
\text { design, training, publications, and } \\
\text { operational procedures to different } \\
\text { cultures. The results of the } \\
\text { research should also be used to } \\
\text { identify significant vulnerabilities, } \\
\text { if any, in existing flight deck } \\
\text { designs, training, or operations, } \\
\text { and how those vulnerabilities } \\
\text { should be addressed. }\end{array}$ & Culture-1 \\
\hline $\begin{array}{c}\text { Cultural and Language } \\
\text { Differences }\end{array}$ & $\begin{array}{l}\text { The FAA should encourage } \\
\text { simplified flight deck messages, } \\
\text { training, manuals, and procedures } \\
\text { with clearer meaning to non-native } \\
\text { English speakers. The FAA should } \\
\text { encourage the use of } \\
\text { internationally understood visual } \\
\text { symbols and pictures where } \\
\text { appropriate, rather than verbal } \\
\text { descriptions or directions. }\end{array}$ & Culture-2 \\
\hline
\end{tabular}




\begin{tabular}{|c|l|c|}
\hline Issue & \multicolumn{1}{|c|}{ Recommendation } & $\begin{array}{c}\text { Recommendation } \\
\text { Number }\end{array}$ \\
\hline $\begin{array}{c}\text { Cultural and Language } \\
\text { Differences }\end{array}$ & $\begin{array}{l}\text { The FAA should provide } \\
\text { leadership to update ICAO } \\
\text { phraseology standards and to } \\
\text { encourage their use. }\end{array}$ & Culture-3 \\
\hline Cultural and Language & $\begin{array}{l}\text { The FAA should promote timely } \\
\text { and clear communications between } \\
\text { flightcrews and Air Traffic } \\
\text { Services through: } \\
\text { Accelerated efforts for } \\
\text { transmission of information via } \\
\text { datalink, as appropriate (e.g., } \\
\text { Automatic Terminal } \\
\text { Information Service (ATIS), } \\
\text { weather, pre-departure } \\
\text { clearances (PDC)); } \\
\text { Assuring clear and intelligible } \\
\text { transmission of ATIS and } \\
\text { clearance information where } \\
\text { datalink is unavailable or } \\
\text { unsuitable; and } \\
\text { Standard procedures and taxi } \\
\text { routes. }\end{array}$ & \\
\hline
\end{tabular}




\section{Appendix C Supporting Data and References}

\section{Books}

Gras, Alain, Caroline Moricot, Sophie L. Poirot-Delpech, and Victor Scardigli. Faced with Automation. The pilot, the controller, the engineer. Paris, France: Publications de la Sorbonne, 1994.

Hofstede, G. Culture's Consequences: International Differences in Work-Related Values. Beverly Hills, California. Sage, 1980.

Wiener, Earl, Barbara G. Kanki, and Robert L. Helmreich, ed. Cockpit Resource Management. San Diego, California: Academic Press, 1993.

Wiener, Earl and David C. Nagel, ed. Human Factors in Aviation. San Diego, California: Academic Press, 1988.

Wise, John A., V. David Hopkin, and Daniel J. Garland, ed. Human Factors Certification of Advanced Aviation Technologies. Daytona Beach, Florida: Embry-Riddle Aeronautical University Press, 1994.

Woods, David D., Leila J. Johannesen, Richard I. Cook, and Nadine B. Sarter. Behind Human Error: Cognitive Systems, Computers and Hindsight. CSERIAC SOAR 94-01, December, 1994.

\section{$\underline{\text { Magazine/Newspaper Articles }}$}

Blattner, Les. "FOQA Takes Off.” Air Line Pilot. November/December 1995, p. 41.

Bresley, Bill. “777 Flight Deck Design.” Boeing Airliner. April-June, 1995, p. 1.

Carbaugh, Captain Dave and Captain Skip Cooper. "Avoiding Controlled Flight into Terrain.” Boeing Airliner. April-June, 1996, p. 1.

Covault, Craig. "A310 Pitches Up, Dives on Orly Approach.” Aviation Week and Space Technology.

Demosthenes, F/O T.A. and Capt. J.G. Oliver. “A Pilot's Perspective." Air Line Pilot. June, 1991, p 22.

Donoghue, J. A. “Making Automation Behave.” Air Transport World. March, 1995, p. 5. 
Donoghue, J. A. “Keepin' the Shiny Side Up.” Air Transport World. October, 1995, p. 47.

Dornheim, Michael A. "Dramatic Incidents Highlight Mode Problems in Cockpits." Aviation Week and Space Technology. January 30, 1995, p. 56.

Dornheim, Michael A. "Modern Cockpit Complexity Challenges Pilot Interfaces." Aviation Week and Space Technology. January 30, 1995, p. 60.

"FAA Gains Access to Flight Data Recorder Information for Safety Purposes." Aviation Daily. February 10, 1995.

Flint, Perry. “A Common Problem.” Air Transport World. March, 1995, p. 51.

Hughes, David. “Aiding Mode Awareness." Aviation Week and Space Technology. February 6, 1995, p. 52.

Hughes, David. "Former NASA Ames Experts Hold Key Airline Posts." Aviation Week and Space Technology. February 6, 1995, p. 52.

Hughes, David. "Gulfstream Using Vertical Profile Display." Aviation Week and Space Technology. February 6, 1995, p. 55.

Hughes, David. "Incidents Reveal Mode Confusion." Aviation Week and Space Technology. January 30, 1995, p. 56.

Hughes, David. "Laptop FMS 'Tutor' Aids Automation Training.” Aviation Week and Space Technology. February 20, 1995, p. 39.

Hughes, David. "Studies Highlight Automation 'Surprises'." Aviation Week and Space Technology. February 6, 1995, p. 48.

Hughes, David. "CRM Library to Help Share Data, Save Money" Aviation Week and Space Technology. June 12, 1995, p. 161.

Hughes, David and Michael A. Dornheim. "Accidents Direct Focus on Cockpit Automation.” Aviation Week and Space Technology. January 30, 1995, p. 52.

Learmount, David. "Lessons from the Cockpit." Flight International. January 17, 1995, p. 24.

Learmount, David. “Off Target.” Flight International. January 17-23, 1996, p. 24. 
Morrocco, John D. "Cockpit Automation Raises Training Concerns." Aviation Week and Space Technology. March 4, 1996, p. 39.

Nordwall, Bruce D. "Military Cockpits Keep Autopilot Interface Simple.” Aviation Week and Space Technology. February 6, 1995, p. 54.

North, David. "Pilots Union Presses for Improved Displays." Aviation Week and Space Technology. February 6, 1995, p. 50.

Orasanu, Judith. "Expert Decision Making on the Flight Deck." FAA Aviation News. September 1994, p 5.

Ott, James. "Safety Coalition Pushes Early Alert System." Aviation Week and Space Technology. January 22, 1996, p. 40.

Ott, James. "United FOQA Data Sheds Light on Airport Approaches." Aviation Week and Space Technology. April 22, 1996, p. 51.

Phillips, Don. "Back to School for Pilots; United Plans a Curriculum With New Ideas About Handling Emergencies." Washington Post. February 3, 1995.

Phillips, Don and Richard M. Weintraub. "Puzzling String of USAir Crashes Puts Spotlight on Human Factors.” Washington Post. September 19, 1994, p A4.

Phillips, Edward H. "Pitot System Errors Blamed in 757 Crash." Aviation Week and Space Technology. March 25, 1996, p. 30.

Phillips, Edward H. "Data Recorders Crucial to State-of-Art Crash Probes." Aviation Week and Space Technology. February 6, 1995, p. 56.

Phillips, Edward H. "FAA to Study Human Factors." Aviation Week and Space Technology. October 24, 1994, p. 20.

Phillips, Edward H. "NTSB: Mode Confusion Poses Safety Threat." Aviation Week and Space Technology. January 30, 1995, p. 63.

Phillips, Edward H. "Battelle Proposal Key to Safety Data Exchange." Aviation Week and Space Technology. February 12, 1996, p. 37.

“Pilots, Airbus Clash in CAL Hearing.” Japan Times. February 8, 1995.

Proctor, Paul. "Upset Training Gains New Role in Light Jets." Aviation Week and Space Technology. February 13, 1995, p. 57. 
Rankin, Bill, Ph.D. and Jerry Allen. "Boeing Introduces Maintenance Error Decision Aid." Boeing Airliner. April-June, 1996, p. 20.

Rosenthal, Loren J., Capt. Roy W. Chamberlin, and S/O Robert D. Matchette. "Flight Deck Confusion.” Air Line Pilot. May, 1996, p. 10.

Scott, William B. "Certification Officials Grapple with Flight Deck Complexity." Aviation Week and Space Technology. January 30, 1995, p. 64.

Scott, William B. "United Training Stresses Cockpit Discipline." Aviation Week and Space Technology. February 6, 1995, p. 50.

Scott, William B. "United Pilots Practice Advanced Maneuvers." Aviation Week and Space Technology. March 27, 1995, p. 42.

Sekigawa, Eiichiro. "Japanese Quiz Airbus on A300 Autopilot.” Aviation Week and Space Technology. February 13, 1995, p. 32.

Shifrin, Carole A. "SAS Launches 737-600 Program." Aviation Week and Space Technology. March 20, 1995, p. 28.

Shifrin, Carole A. "U.K. Panel Urges Review of A340 Management Systems." Aviation Week and Space Technology. March 20, 1995, p. 34.

Sparaco, Pierre. “Airbus Seeks to Keep Pilot, New Technology in Harmony.” Aviation Week and Space Technology. January 30, 1995, p. 62.

Sparaco, Pierre. "French Issue Airbus Alert." Aviation Week and Space Technology. October 24, 1994, p. 21.

Sparaco, Pierre. "Tarom A310 Crash Probe Focuses on Throttle Failure.” Aviation Week and Space Technology. April 17, 1995, p. 41.

"Training on Unusual Attitude/Loss of Control Added to FlightSafety Courses." Aviation Daily. January 30, 1995.

“U.S. Airlines Delay Exchange of Safety Data." Aviation Week and Space Technology. January 29, 1996, p. 51.

Walley, Susanne. "Training of the Commercial Airline Pilot" World Aerospace Technology -- The International Review of Aerospace Design and Development. 1994.

Wiley, John. "Mastering Autoflight.” Air Transport World. March, 1995, p. 45. 


\section{Incident/Accident Reports}

Airbus Industrie. Interflug Nose Up During Go Around Procedure. Incident Report, April $15,1991$.

Airbus Industrie. A310 Incident in Orly. Telex, November 24, 1994.

Airbus Industrie. A310 incident at Orly. Accident brief, October 14, 1994.

Aviation Safety Reporting System. A-320 Type Reports. February 26, 1992.

Aviation Safety Reporting System. Airbus Reports. November 30, 1994.

Boeing Commercial Airplane Group. Accident Prevention Strategies, Removing Links in the Accident Chain, Commercial Jet Aircraft Accidents, World Wide Operations, 19821991. October, 1993.

Boeing Commercial Airplane Group. Statistical Summary of Commercial Jet Aircraft Accidents, World Wide Operations, 1959-1995. April, 1996.

Commission of Investigation. Preliminary Report, A330 Flight Test Accident. July 28, 1994.

Direction Générale de l'Aviation Civile (French DGAC). A320 - Dragonair Flap Event in Hong Kong. Preliminary findings, July 22, 1994.

Direction Générale de l'Aviation Civile (French DGAC). Conclusions Drawn from the Report by the Commission of Investigation of the Air Inter Airbus A320 accident on 20th January 1992 close to Mont Sainte-Odile. December 16, 1993.

Douglas Aircraft Company. MD-11 Pitch Upsets in Severe Turbulence. All Operators Letter, July 1, 1994.

Douglas Aircraft Company. MD-11 Inadvertent Autopilot Disconnect. All Operators Letter, July 5, 1994.

Douglas Aircraft Company. Commercial Jet Transport Safety Statistics, 1993. Undated.

National Transportation Safety Board. Stall and Loss of Control on Final Approach, Atlantic Coast Airlines, Inc./United Express Flight 6291, Jetstream 4101, N304UE, Columbus, Ohio, January 7, 1994. Accident Report, October 6, 1994.

National Transportation Safety Board. A Review of Flightcrew-Involved, Major Accidents of U.S. Air Carriers, 1978 Through 1990. Safety Study, January, 1994. 


\section{Study Reports, Bulletins, Scientific Journal Articles}

Air Line Pilots Association. Study Group Recommendations, Meeting with Airbus. Report of the North American A-320 Study Group, June 15, 1994.

Aviation Safety Plan. Published by the U.S. Department of Transportation Federal Aviation Administration, February, 1996.

Billings, Charles E. Human-Centered Automation: A Concept and Guidelines. NASA Technical Memorandum 103885, August, 1991.

Billings, Charles E. Human-Centered Automation: Principles and Guidelines. NASA Technical Memorandum, March, 1995.

Boje, E. and T. Hancke. Summary of Panel Discussion: Automation in Commercial Aviation. Undated

Bureau of Air Safety Investigation (Australia Department of Transport). Advanced Technology Aircraft Project Phase 1 -- An Information Paper of the Emerging Safety Issues. SAB/IP/94/01, RP/91/04, April, 1994.

Bureau of Air Safety Investigation (Australia Department of Transport). Research Proposal -- Advance Technology Aircraft -- Phase Two. undated.

Cardosi, Kim M. and M. Stephen Huntley. Human Factors for Flight Deck Certification Personnel. DOT/FAA/RD-93/5 Technical Report, July, 1993.

Corwin, William H. Investigation of Operational Problems Associated with Flight Mode Annunciation. NASA Grant (NAG1-1581), December 15, 1995.

Degani, Asaf. On the Typography of Flight-Deck Documentation. NASA Contractor Report, December, 1992.

Degani, Asaf and Earl L. Wiener. On the Design of Flight Deck Procedures. NASA Contractor Report, June, 1994.

Degani, Asaf and Earl L. Wiener. Human Factors of Flight Deck Checklists: The Normal Checklist. NASA Contractor Report, May, 1990.

Fadden, Delmar M. Error Tolerant Avionics and Displays. October 25, 1989.

Flight Crew Training on the Hazard of Attempting to Counter Auto Pilot Commands by Manual Control Forces in the Airbus A-310, and A-300-600 Series Airplane. Flight Standards Information Bulletin for Air Transportation, draft. 
Folkerts, H.H., and P.G.A.M. Jorna, Pilot Performance in Automated Cockpits: A Comparison of Moving and Non-Moving Thrust Levers. National Aerospace Laboratory of the Netherlands, NLR TP 94005 U, January 14, 1994.

Funk, Ken, Beth Lyall, and Vic Riley. A Comparative Analysis of Flightdecks With Varying Levels of Automation. FAA grant, phase 1 progress report, August 2, 1994.

Funk, Ken, Beth Lyall, and Vic Riley. Perceived Human Factors Problems of Flightdeck Automation. FAA grant, phase 1 final report, December, 1995.

Harrison, L., J. Janowitz, and M. Castronuovo. Pilot-Vehicle Interface. DOT/FAA/CT92/21 Technical Report. November, 1993.

Helmreich, R.L, A.C. Merritt, and P.J. Sherman. The Flight Management Attitudes Questionnaire: An international survey of pilot attitudes regarding cockpit management and automation. Study in progress. Austin, Texas: NASA/University of Texas/FAA Aerospace Crew Research Project, 1996.

ICARUS Committee, The Dollars and Sense of Risk Management and Airline Safety. Flight Safety Foundation. Flight Safety Digest. December 1994.

Last, Steve, Martin Alder. British Airways Airbus A320 Pilots' Autothrust Survey. SAE Technical Paper Series, 912225, 1991.

Merritt, A.C. and R.L. Helmreich. "Human Factors on the Flight Deck: The Influence of National Culture.” Journal of Cross Cultural Psychology. 1996.

Merritt, A.C. and R.L. Helmreich. "Attitudes Toward Automation: The Effect of National Culture." Proceedings of the Eighth International Symposium on Aviation Psychology. In press.

National Plan for Civil Aviation Human Factors: An Initiative for Research and Application. Sponsored by the Federal Aviation Administration. February 1995.

Nelson, William R., James C. Byers, Lon N. Haney, Lee T. Ostrom, and Wendy J. Reece. Human Error Analysis of ASRS Reports: Altitude Deviations in Advanced Technology Aircraft. Conducted for the National Aeronautics and Space Administration, November, 1992.

Palmer, Michael T., William H. Rogers, Hayes N. Press, Kara A. Latorella, and Terence S. Abbott. A Crew-Centered Flight Deck Design Philosophy for High-Speed Civil Transport (HSCT) Aircraft. NASA Technical Memorandum 109171. January, 1995.

Proceedings of the FAA Office of System Safety Workshop on Flight Crew Caused Accident and Incident Human Factors. June 21-23, 1995. 
Pinet, Jean and John H. Enders. Human Factors in Aviation: A Consolidated Approach. Flight Safety Foundation. Flight Safety Digest. December 1994.

Rosenthal, Loren J., Roy W. Chamberlin, and Robert D. Matchette. "Flight Deck Confusion Cited in Many Aviation Incident Reports." Human Factors \& Aviation Medicine. July-August, 1994.

The Royal Aeronautical Society and The Guild of Air Pilots and Air Navigators. The Future Flight Deck. Discussion paper, Undated.

Rudisill, Marianne. "Flight Crew Experience with Automation Technologies on Commercial Transport Flight Decks." Human Performance in Automated Systems: Current Research \& Trends. 1994.

Sarter, Nadine B. and David D. Woods. "Pilot Interaction With Cockpit Automation: Operational Experiences With the Flight Management System." The International Journal of Aviation Psychology, 2(4), p 303-321. 1992.

Sarter, Nadine B. and David D. Woods. "Pilot Interaction with Cockpit Automation II: An Experimental Study of Pilots' Model and Awareness of the Flight Management System (FMS)." The International Journal of Aviation Psychology, 4(1), p 1-28. 1994.

Sarter, Nadine B. and David D. Woods. "Strong, Silent, And 'Out-of-the-Loop"”: Properties of Advanced (Cockpit) Automation and Their Impact On Human-Automation Interaction. Ohio State University Cognitive Systems Engineering Laboratory Report 95TR-01, February 1995.

Sarter, Nadine B. and David D. Woods. 'How in the world did we ever get into that mode' Mode Error and Awareness in Supervisory Control. Human Factors, 37(1), 5-19, 1995.

Speyer, Jean Jacques. Cockpit Design-Induced Error Tolerance. Paper delivered at the 9th Orient Airlines Association, 13-14 May 1987.

Sumwalt, Robert L. III. "Accident and Incident Reports Show Importance of 'Sterile Cockpit' Compliance.” Flight Safety Digest. July, 1994.

Wiener, Earl L. Human Factors of Advanced Technology ("Glass Cockpit") Transport Aircraft. NASA Contractor Report 1775281989.

Wiener, Earl L. Flight Deck Automation: Promises and Realities. NASA Conference Publication 10036, 1989. 
Wiener, Earl L. Intervention Strategies for the Management of Human Error. NASA Contractor Report, August, 1993.

Wiener, Earl L. Human Factors of Cockpit Automation: A Field Study of Flight Crew Transition. NASA Contractor Report, July, 1985.

Zebedee, Doug. Altitude Acquire Mode Safety Bulletin, October 26, 1994.

\section{$\underline{\text { Presentations }}$}

Abbott, Kathy. Aviation Safety/Automation. January 10, 1995.

Abbott, Terence S. Designing for the User: Task-Oriented Display Design. Undated (presented January 10, 1995).

Abbott, T. and W. Rogers. Cockpit Integration Technology. September 10, 1990.

Airbus Industrie. A300-600 Accident at Nagoya on April 26, 1994. August, 1994.

Airbus Industrie. A310-300 Incident at Paris - Orly on September 24, 1994. August, 1994.

Airbus Industrie. Narrow Body Family. Man-Machine Interface Improvements Done Upon Airline Request. 1995.

Amalberti, R. Harmonization Working Group and other Human Factors Groups. What Relationships? October, 1994.

Billings, Charles E. Some Thoughts on Advanced Aircraft Automation. December 7, 1994.

Ekstrand, Capt. Chet. Managing the Modern Cockpit - The Manufacturer's View. Undated (presented April 7, 1995).

FAA Human Factors Team Review at BCAG. April 6-7, 1995.

FAA Human Factors Team Visit to Fokker Aircraft Company. Review of the Fokker Jetline Flight Deck Automation. May 8 and 9, 1995.

Graeber, R. Curtis, Ph.D. Presentation on SOW 1.6: Human Error. October 13, 1994.

Hansman, R. John, Alan Midkiff, Sanjay Vakil, Thomas Vaneck, William Corwin, Sharon Irving, Jim Irving, and Peter Polson. Mode Awareness In Advanced AutoFlight Systems. Undated (presented January 11, 1995). 
Interfaces Between Flight Crews and Modern Flight Deck Systems. FAA/JAA Human Factors Team Visit to Airbus Industrie. May 3-5, 1995.

Ice Plan of Fokker Aircraft. Presentation to FAA Human Factors Team. May, 1995.

James, M., A. McClumpha, R. Green, P. Wilson, and A. Belyavin. Pilot Attitudes to Flight Deck Automation. March 14, 1991.

Kelly, Brian D., R. Curtis Graeber, and Delmar M. Fadden. Applying Crew-centered Concepts to Flight Deck Technology: The Boeing 777. Flight Safety Foundation 45th International Air Safety Seminar and the 22nd International Conference of the International Federation of Airworthiness. 1992.

Last, Capt. Steve. “Crew-Caused” Accidents: Some Hidden Origins. December, 1994.

McClumpha, A.J., M. James, R.G. Green, and A.J. Belyavin. Pilots' Attitudes to Cockpit Automation. 1991.

Palmer, Dr. Everett A. Aviation Safety/Automation Research Summary. January 11, 1995.

Palmer, Michael T., Peter Polson, Sharon Irving, Jim Irving, Bill Corwin, John Hansman, Alan Midkiff, and Sanjay Vakil. Autoflight System Mode Awareness and Mode Control Panel Redesign. Undated (presented January 11, 1995).

Polson, Peter G. and Asaf Degani. Flight Mode Annunciation: Solution or Problem. Undated (presented January 11, 1995).

Rudisill, Dr. Marianne. Analysis of International Automation Survey Comments. January 10, 1995.

Sarter, Nadine B. and David Woods. Strong, Silent, and Difficult to Direct: Why Advanced (Cockpit) Automation is not a Team Player. December 1994.

Schutte, Paul. Intelligent Error Tolerant Systems. Undated (presented January 10, 1995).

Tenney, Yvette J., William H. Rogers, and Richard W. Pew. Pilot Opinions on High Level Flight Deck Automation Issues: Toward the Development of a Design Philosophy. Undated (presented January 11, 1995).

Weener, Earl and Paul D. Russell. Aviation Safety Overview. Flight Safety Foundation 46th International Air Safety Seminar and the 23rd International Conference of the International Federation of Airworthiness. November 8-11, 1993. 


\section{Appendix D \\ Examples of Incidents and Accidents Involving The Flightcrew-Automation Interface}

\begin{tabular}{|c|c|c|c|c|}
\hline Date & Location & $\frac{\text { Airplane }}{\text { Type }}$ & Operator & Description \\
\hline $12 / 29 / 72$ & Miami & L-1011 & $\begin{array}{l}\text { Eastern Air } \\
\text { Lines }\end{array}$ & $\begin{array}{l}\text { Flightcrew members became immersed in } \\
\text { an apparently malfunctioning landing gear. } \\
\text { Airplane was in control wheel steering } \\
\text { mode. Altitude hold inadvertently } \\
\text { disengaged by a light force on the control } \\
\text { wheel. Altitude alert aural warning not } \\
\text { heard by flightcrew. Fatal crash. }\end{array}$ \\
\hline $7 / 31 / 73$ & Boston & DC-9-31 & $\begin{array}{l}\text { Delta Air } \\
\text { Lines }\end{array}$ & $\begin{array}{l}\text { Airplane landed short during an approach in } \\
\text { fog. Flightcrew was preoccupied with } \\
\text { questionable information presented by the } \\
\text { flight director. Fatal crash. }\end{array}$ \\
\hline $2 / 28 / 84$ & New York & DC-10-30 & $\begin{array}{l}\text { Scandinavian } \\
\text { Airlines }\end{array}$ & $\begin{array}{l}\text { Malfunctioning autothrottle system during } \\
\text { approach resulted in crossing the runway } \\
\text { threshold at } 50 \text { knots above reference } \\
\text { speed. Runway was wet, touchdown was } \\
4700 \text { feet beyond the threshold of an } 8400 \\
\text { foot runway. Airplane overran runway, } \\
\text { minor injuries. Complacency and over- } \\
\text { reliance on automatic systems cited. }\end{array}$ \\
\hline $2 / 19 / 85$ & $\begin{array}{l}\text { San } \\
\text { Francisco }\end{array}$ & 747SP & $\begin{array}{l}\text { China } \\
\text { Airlines }\end{array}$ & $\begin{array}{l}\text { Loss of power on one engine during } \\
\text { autoflight. Autopilot tried to compensate } \\
\text { until control limits were reached. Captain } \\
\text { disengaged autopilot, airplane went into } \\
\text { unusual attitude high speed dive, but was } \\
\text { successfully recovered. Autopilot masked } \\
\text { approaching onset of loss of control. }\end{array}$ \\
\hline
\end{tabular}




\begin{tabular}{|c|c|c|c|c|}
\hline Date & Location & $\begin{array}{l}\text { Airplane } \\
\text { Type }\end{array}$ & Operator & Description \\
\hline $6 / 26 / 88$ & Habsheim & A 320 & Air France & $\begin{array}{l}\text { Low, slow flyover at air show. Ran out of } \\
\text { energy and flew into trees. Possible } \\
\text { overconfidence in the envelope protection } \\
\text { features of the A } 320 \text {. Fatal crash. }\end{array}$ \\
\hline $7 / 3 / 88$ & Gatwick & A320 & unknown & $\begin{array}{l}\text { Programmed for } 3 \text { degree flight path, but } \\
\text { inadvertently was in vertical speed mode, } \\
\text { almost landed } 3 \text { miles short. }\end{array}$ \\
\hline $1 / 89$ & Helsinki & A300 & KAR Air & $\begin{array}{l}\text { While making an ILS approach, the } \\
\text { takeoff/go-around lever was inadvertently } \\
\text { depressed. In response to the unexpected } \\
\text { and sudden nose-up change in the airplane's } \\
\text { attitude, the flightcrew immediately reacted } \\
\text { by re-trimming. }\end{array}$ \\
\hline $6 / 8 / 89$ & Boston & 767 & unknown & $\begin{array}{l}\text { On autopilot ILS approach, airplane } \\
\text { overshot the localizer. Captain switched } \\
\text { from approach to heading select mode to } \\
\text { regain the localizer, disengaged the } \\
\text { autopilot, and used the flight director. Since } \\
\text { the glide slope had not been captured, the } \\
\text { flight director was in vertical speed mode } \\
\text { commanding an } 1,800 \text { fpm rate of descent. } \\
\text { Alert from the ground proximity warning } \\
\text { and tower resulted in a go-around from } \\
\text { about } 500 \text { feet. }\end{array}$ \\
\hline $2 / 14 / 90$ & Bangalore & A320 & $\begin{array}{l}\text { Indian } \\
\text { Airlines }\end{array}$ & $\begin{array}{l}\text { Inappropriate use of open descent mode. } \\
\text { Fatal crash. }\end{array}$ \\
\hline $6 / 90$ & San Diego & A320 & unknown & $\begin{array}{l}\text { Pilot mistakenly set vertical speed of } 3,000 \\
\text { fpm instead of } 3.0 \text { degree flight path angle. } \\
\text { Error was caught, but airplane descended } \\
\text { well below profile and minimum descent } \\
\text { altitude. }\end{array}$ \\
\hline
\end{tabular}




\begin{tabular}{|c|c|c|c|c|}
\hline Date & Location & $\begin{array}{l}\text { Airplane } \\
\text { Type }\end{array}$ & Operator & Description \\
\hline $2 / 11 / 91$ & Moscow & A 310 & Interflug & $\begin{array}{l}\text { Pilot intervention in auto-pilot coupled go- } \\
\text { around resulted in the autopilot } \\
\text { commanding nose-up trim while the pilot } \\
\text { was applying nose-down elevator. } \\
\text { Autopilot disconnected when mode } \\
\text { transitioned to altitude acquire mode - force } \\
\text { disconnect not inhibited in this mode as it is } \\
\text { in go-around mode. Airplane ended up } \\
\text { badly out of trim and went through several } \\
\text { extreme pitch oscillations before the } \\
\text { flightcrew regained control. }\end{array}$ \\
\hline $1 / 20 / 92$ & Strasbourg & A320 & Air Inter & $\begin{array}{l}\text { Evidence suggests flightcrew inadvertently } \\
\text { selected } 3,300 \text { fpm descent rate on } \\
\text { approach instead of } 3.3 \text { degree flight path } \\
\text { angle. Fatal crash. }\end{array}$ \\
\hline $9 / 14 / 93$ & Warsaw & A320 & Lufthansa & $\begin{array}{l}\text { Wet runway, high tailwinds -- After } \\
\text { touchdown, the air/ground logic did not } \\
\text { indicate the airplane was on the ground, } \\
\text { and delayed deployment of ground spoilers } \\
\text { and reversers. Airplane overran runway. } \\
\text { Two fatalities. }\end{array}$ \\
\hline
\end{tabular}




\begin{tabular}{|c|c|c|c|c|}
\hline Date & Location & $\begin{array}{l}\text { Airplane } \\
\text { Type }\end{array}$ & Operator & Description \\
\hline $9 / 13 / 93$ & Tahiti & $747-400$ & Air France & $\begin{array}{l}\text { VNAV approach with autothrottle } \\
\text { engaged, autopilot disengaged. Upon } \\
\text { reaching the published missed approach } \\
\text { point, VNAV commanded a go-around and } \\
\text { the autothrottle advanced power. After a } \\
\text { delay, the flightcrew manually reduced } \\
\text { power to idle and held the thrust levers in } \\
\text { the idle position. The airplane landed long } \\
\text { and fast. Two seconds prior to touchdown } \\
\text { the number one engine thrust lever } \\
\text { advanced to nearly full forward thrust and } \\
\text { remained there until the airplane stopped. } \\
\text { Reverse thrust was obtained on the other } \\
\text { engines. The spoilers were not deployed -- } \\
\text { the automatic system did not operate } \\
\text { because the number one thrust lever was } \\
\text { not at idle, and the flightcrew did not } \\
\text { extend them manually. The flightcrew lost } \\
\text { directional control of the airplane as the } \\
\text { speed decreased and the airplane went off } \\
\text { the right side of the runway. }\end{array}$ \\
\hline
\end{tabular}




\begin{tabular}{|c|c|c|c|c|}
\hline Date & Location & $\begin{array}{l}\text { Airplane } \\
\text { Type }\end{array}$ & Operator & Description \\
\hline 6/6/94 & Hong Kong & A 320 & Dragonair & $\begin{array}{l}\text { After three missed approaches due to } \\
\text { lateral oscillations in turbulent conditions, a } \\
\text { landing was made and the airplane went off } \\
\text { the side of the runway. The flaps locked at } \\
40 \text { degrees deflection (landing position) just } \\
\text { before the first go-around due to } \\
\text { asymmetry. Asymmetry caused by rigging } \\
\text { at the design tolerance combined with gust } \\
\text { loads experienced. In accordance with } \\
\text { published procedures, flightcrew selected } \\
\text { CONF } 3 \text { for landing, which extended slats } \\
\text { to } 22 \text { degrees. With autopilot engaged, } \\
\text { lateral control laws correspond to control } \\
\text { lever position. Under manual control, } \\
\text { control laws correspond to actual flap/slat } \\
\text { position. The configuration CONF } 3 \text {, with } \\
\text { flaps locked at } 40 \text { degrees, is more } \\
\text { susceptible to lateral oscillations with the } \\
\text { autopilot engaged. After a similar incident } \\
\text { in November, } 1993 \text {, experienced by Indian } \\
\text { Airlines, Airbus issued an Operations } \\
\text { Engineering Bulletin to leave the control } \\
\text { lever in CONF FULL if the flaps lock in } \\
\text { that position. }\end{array}$ \\
\hline
\end{tabular}




\begin{tabular}{|c|c|c|c|c|}
\hline Date & Location & $\begin{array}{l}\text { Airplane } \\
\text { Type }\end{array}$ & Operator & Description \\
\hline $4 / 26 / 94$ & Nagoya & A $300-600$ & $\begin{array}{l}\text { China } \\
\text { Airlines }\end{array}$ & $\begin{array}{l}\text { Flightcrew inadvertently activated the go- } \\
\text { around switches on the throttle levers } \\
\text { during a manually flown approach. This } \\
\text { action engaged the autothrottles and put } \\
\text { the flight guidance system in go-around } \\
\text { mode. Flightcrew disconnected the } \\
\text { autothrottles, but excess power caused } \\
\text { divergence above the glide slope. } \\
\text { Flightcrew attempted to stay on glide slope } \\
\text { by commanding nose-down elevator. The } \\
\text { autopilot was then engaged, which because } \\
\text { it was still in go-around mode, commanded } \\
\text { nose-up trim. Flightcrew attempted go- } \\
\text { around after "alpha floor" protection was } \\
\text { activated, but combination of out-of-trim } \\
\text { condition, high engine thrust, and retracting } \\
\text { the flaps too far led to a stall. Fatal crash. }\end{array}$ \\
\hline $6 / 21 / 94$ & Manchester & $757-200$ & Britannia & $\begin{array}{l}\text { Altitude capture mode activated shortly } \\
\text { after takeoff, autothrottles reduced power, } \\
\text { flight director commanded pitch-up before } \\
\text { disappearing. Airspeed dropped toward } \mathrm{V}_{2} \\
\text { before flightcrew pitched the nose down to } \\
\text { recover. }\end{array}$ \\
\hline $6 / 30 / 94$ & Toulouse & A330 & Airbus & $\begin{array}{l}\text { Unexpected mode transition to altitude } \\
\text { acquire mode during a simulated engine } \\
\text { failure resulted in excessive pitch, loss of } \\
\text { airspeed, and loss of control. Pitch attitude } \\
\text { protection not provided in altitude acquire } \\
\text { mode. Fatal crash. }\end{array}$ \\
\hline
\end{tabular}




\begin{tabular}{|c|c|c|c|c|}
\hline Date & Location & $\begin{array}{l}\text { Airplane } \\
\text { Type }\end{array}$ & Operator & Description \\
\hline $9 / 24 / 94$ & Paris - Orly & A310-300 & Tarom & $\begin{array}{l}\text { Overshoot of flap placard speed during } \\
\text { approach caused a mode transition to flight } \\
\text { level change. Autothrottles increased power } \\
\text { and trim went full nose-up for unknown } \\
\text { reasons (autopilot not engaged). Flightcrew } \\
\text { attempted to stay on path by commanding } \\
\text { nose-down elevator, but could not } \\
\text { counteract effect of stabilizer nose-up trim. } \\
\text { Airplane stalled, but was recovered. }\end{array}$ \\
\hline $10 / 31 / 94$ & Roselawn & ATR-72 & $\begin{array}{l}\text { American } \\
\text { Eagle }\end{array}$ & $\begin{array}{l}\text { In a holding pattern, the airplane was } \\
\text { exposed to a complex and severe icing } \\
\text { environment, including droplet sizes much } \\
\text { larger than those specified in the } \\
\text { certification requirements for the airplane. } \\
\text { During a descending turn immediately after } \\
\text { the flaps were retracted, the ailerons } \\
\text { suddenly deflected in the right-wing down } \\
\text { direction, the autopilot disconnected, and } \\
\text { the airplane entered an abrupt roll to the } \\
\text { right. The flightcrew were unable to correct } \\
\text { this roll before the airplane impacted the } \\
\text { ground. }\end{array}$ \\
\hline $3 / 31 / 95$ & Bucharest & A310-300 & Tarom & $\begin{array}{l}\text { Shortly after takeoff in poor visibility and } \\
\text { heavy snow, with autothrottles engaged, } \\
\text { climb thrust was selected. The right engine } \\
\text { throttle jammed and remained at takeoff } \\
\text { thrust, while the left engine throttle slowly } \\
\text { reduced to idle. The increasing thrust } \\
\text { asymmetry resulted in an increasing left } \\
\text { bank angle, which eventually reached about } \\
170 \text { degrees. The airplane lost altitude and } \\
\text { impacted the ground at an } 80 \text {-degree angle. } \\
\text { Only small rudder and elevator deflections } \\
\text { were made until seconds before impact, } \\
\text { when the left throttle was brought back to } \\
\text { idle to remove the thrust asymmetry. Fatal } \\
\text { crash. }\end{array}$ \\
\hline
\end{tabular}




\begin{tabular}{|c|c|c|c|c|}
\hline Date & Location & $\frac{\text { Airplane }}{\text { Type }}$ & Operator & Description \\
\hline $11 / 12 / 95$ & $\begin{array}{l}\text { Bradley } \\
\text { International } \\
\text { Airport }\end{array}$ & MD-80 & $\begin{array}{l}\text { American } \\
\text { Airlines }\end{array}$ & $\begin{array}{l}\text { On a VOR-DME approach, the airplane } \\
\text { descended below the minimum descent } \\
\text { altitude, clipped some trees, and landed } \\
\text { short of the runway. Contributing to this } \\
\text { incident was a loss of situation awareness } \\
\text { and terrain awareness by the flightcrew, } \\
\text { lack of vertical guidance for the approach, } \\
\text { and insufficient communication and } \\
\text { coordination by the flightcrew. }\end{array}$ \\
\hline $12 / 20 / 95$ & Cali & $757-200$ & $\begin{array}{l}\text { American } \\
\text { Airlines }\end{array}$ & $\begin{array}{l}\text { Unexpectedly cleared for a direct approach } \\
\text { to Cali, the flightcrew apparently lost } \\
\text { situation awareness and crashed into a } \\
\text { mountain north of the city. On approach, } \\
\text { the flightcrew were requested to report } \\
\text { over Tulua VOR. By the time this waypoint } \\
\text { was input into the flight management } \\
\text { computer, the airplane had already flown } \\
\text { past it; the autopilot started a turn back to } \\
\text { it. The flightcrew intervened, but the course } \\
\text { changes put them on a collision course with } \\
\text { a mountain. Although the ground proximity } \\
\text { warning system alerted the flightcrew, and } \\
\text { the flightcrew responded, they neglected to } \\
\text { retract the speedbrakes and were unable to } \\
\text { avoid hitting the mountain. Fatal crash. }\end{array}$ \\
\hline
\end{tabular}




\begin{tabular}{|c|c|c|c|c|}
\hline Date & Location & $\begin{array}{l}\text { Airplane } \\
\text { Type }\end{array}$ & Operator & Description \\
\hline 2/6/96 & Puerto Plata & $757-200$ & Birgenair & $\begin{array}{l}\text { After taking off from Puerto Plata, the } \\
\text { flightcrew lost control of the airplane } \\
\text { during climb and crashed into the ocean off } \\
\text { the coast of the Dominican Republic. } \\
\text { Problems with the captain's airspeed } \\
\text { indication were encountered during the } \\
\text { takeoff roll, and the takeoff and initial } \\
\text { climbout were conducted using airspeed } \\
\text { callouts by the first officer. Continued } \\
\text { erroneous airspeed indications, possibly due } \\
\text { to a blocked pitot tube, resulted in an } \\
\text { overspeed warning during climb. Shortly } \\
\text { thereafter the stickshaker activated. The } \\
\text { conflicting warnings (overspeed and stall) } \\
\text { apparently confused the flightcrew. The } \\
\text { airplane entered a stall from which it did } \\
\text { not recover. Fatal crash. }\end{array}$ \\
\hline
\end{tabular}


This page intentionally left blank 


\section{Appendix E Existing FAR Part 25 Regulations and Advisory Circulars Related To Human Factors}

\section{FAR Part 25 Regulations}

\section{Subpart B -- Flight}

Section 25.101(h) states that "The procedures established [for takeoff, landing, changes in the airplane's configuration, speed, power, and thrust, balked landings, and missed approaches] must --

(1) Be able to be consistently executed in service by crews of average skill;

(2) Use methods or devices that are safe and reliable; and

(3) Include allowance for any time delays, in the execution of the procedures, that may reasonably be expected in service."

Section 25.105(b) states that "No takeoff made to determine the data required by this section may require exceptional piloting skill or alertness."

Section 25.109(b)(3) allows a means of deceleration other than wheel brakes to be used to determine the accelerate-stop distance if that means "[i]s such that exceptional skill is not required to control the airplane."

Section 25.125(a)(5) states that "The landings [used to determine the landing distance data] may not require exceptional piloting skill or alertness."

Section 25.125(b)(3) allows a means of deceleration other than wheel brakes to be used to determine the landing distance if that means "[i]s such that exceptional skill is not required to control the airplane."

Section 25.143(b) states that "It must be possible to make a smooth transition from one flight condition to any other flight condition without exceptional piloting skill, alertness or strength...including --

(1) The sudden failure of the critical engine;

(2) For airplanes with three or more engines, the sudden failure of the second critical engine when the airplane is in the en route, approach, or landing configuration and is trimmed with the critical engine inoperative; and

(3) Configuration changes, including deployment or retraction of deceleration devices." 
Section 25.145(c) states that "It must be possible, without exceptional piloting skill, to prevent loss of altitude when complete retraction of the high lift devices from any position is begun during steady, straight level flight..." (Flap gates, which prevent inadvertent movement through the gated position may be used to comply with this requirement using segmented retractions.)

Section 25.149(d) states that "...during recovery [during the flight tests to establish $\mathrm{V}_{\mathrm{MC}}$ ], the airplane may not assume any dangerous attitude or require exceptional piloting skill, alertness, or strength to prevent a heading change of more than 20 degrees."

Section 25.149(e) requires $\mathrm{V}_{\text {MCG }}$ to be determined "using normal piloting skill."

Section 25.149(h) states that "the airplane may not...require exceptional piloting skill, alertness, or strength to prevent a divergence on the approach flight path that would jeopardize continued safe approach [in determining $\mathrm{V}_{\mathrm{MCL}}$ and $\mathrm{V}_{\mathrm{MCL}-2}$ ]..."

Section 25.173(d) allows some neutral static longitudinal stability "if exceptional attention on the part of the pilot is not required to return to and maintain the desired trim speed and altitude."

Section $25.177(\mathrm{~d})$ states that "[t]he dihedral effect...may be negative provided the divergence is gradual, easily recognized, and easily controlled by the pilot."

Section 25.181(b) states that Dutch roll "[m]ust be controllable with normal use of the primary controls without requiring exceptional pilot skill."

Section 25.203(c) states that "For turning flight stalls, the action of the airplane after the stall may not be so violent or extreme as to make it difficult, with normal piloting skill, to effect a prompt recovery and to regain control of the airplane."

Sections 25.207(a) and (b) state that "Stall warning...must be clear and distinctive to the pilot. [A] visual stall warning device that requires the attention of the flightcrew within the cockpit is not acceptable by itself."

Section 25.233 states that "Landplanes must be satisfactorily controllable, without exceptional piloting skill or alertness in...landings..."

Section 25.251(c) states that "...there may be no buffeting condition, in normal flight, including configuration changes during cruise, severe enough to...cause excessive fatigue to the crew..."

Section 25.253(a)(2) states that "Allowing for pilot reaction time after effective inherent or artificial speed warning occurs, it must be shown that the airplane can be recovered to a normal attitude and its speed reduced to $\mathrm{V}_{\mathrm{MO}} / \mathrm{M}_{\mathrm{MO}}$ without --

(i) Exceptional piloting strength or skill..." 


\section{Subpart D -- Design And Construction}

Section 25.671(a) states that "Each control and control system must operate with the ease, smoothness, and positiveness appropriate to its function."

Section 25.671(c) states that "The airplane must be...capable of continued safe flight and landing after any of the following failures or jamming in the flight control system...without requiring exceptional piloting skill or strength. Probable malfunctions must have only minor effects on control system operation and must be capable of being readily counteracted by the pilot."

Section 25.672(a) states that "A warning which is clearly distinguishable to the pilot under expected flight conditions without requiring his attention must be provided for any failure in the stability augmentation system or in any other automatic or power-operated system which could result in an unsafe condition if the pilot were not aware of the failure. Warning systems must activate the control systems."

Section 25.672(b) states that "The design of the stability augmentation system or of any other automatic or power-operated system must permit initial counteraction of failures of the type specified in $\S 25.671$ (c) without requiring exceptional pilot skill or strength, by either the deactivation of the system, or a failed portion thereof, or by overriding the failure by movement of the flight controls in the normal sense."

Sections 25.677(a) and (b) require trim controls "be designed to prevent inadvertent or abrupt operation and to operate in the plane, and with the sense of motion, of the airplane...There must be means adjacent to the trim control to indicate the direction of the control movement relative to the airplane motion. In addition, there must be clearly visible means to indicate the position of the trim device with respect to the range of adjustment."

Section 25.679 requires that gust locks, if when engaged, prevent normal operation of the control surfaces by the pilot, must "automatically disengage when the pilot operates the primary flight controls in a normal manner, or limit the operation of the airplane so that the pilot receives unmistakable warning at the start of takeoff."

Section 25.685(b) states that a means must be provided "in the cockpit to prevent the entry of foreign objects into places where they could jam the [control] system."

Section 25.697 states:

"(a) Each lift device control must be designed so that the pilots can place the device in any takeoff, en route, approach, or landing position...Lift and drag devices must maintain the selected positions except for movement produced by an automatic positioning or load limiting device, without further attention by the pilots.

(b) Each lift and drag device control must be designed and located to make inadvertent operation improbable. Lift and drag devices intended for ground 
operation only must have means to prevent the inadvertent operation of their controls in flight if that operation could be hazardous.

(c) The rate of motion of the surface in response to the operation of the control and the characteristics of the automatic positioning or load limiting device must give satisfactory flight and performance characteristics under steady or changing conditions of airspeed, engine power, and airplane attitude..."

Section 25.699 states:

"(a) There must be means to indicate to the pilots the position of each lift or drag device having a separate control in the cockpit to adjust its position. In addition, an indication of unsymmetrical operation or other malfunction in the lift or drag device systems must be provided when such indication is necessary to enable the pilots to prevent or counteract an unsafe flight or ground condition, considering the effects on flight characteristics and performance.

(b) There must be means to indicate to the pilots the takeoff, en route, approach, and landing lift device positions.

(c) If any extension of the lift and drag devices beyond the landing position is possible, the controls must be clearly marked to identify this range of extension."

Section 25.703 states:

"A takeoff warning system must be installed and must meet the following requirements:

(a) The system must provide to the pilots an aural warning that is automatically activated during the initial portion of the takeoff roll if the airplane is in a configuration...that would not allow a safe takeoff..."

Section 25.729(e) requires "a landing gear position indicator...or other means to inform the pilot that the gear is secured in the [proper] position. The flightcrew must be given an aural warning that functions continuously, or is periodically repeated, if a landing is attempted when the landing gear is not locked down. The warning must be given in sufficient time to allow the landing gear to be locked down or a go-around to be made. There must not be a manual shutoff means readily available to the flightcrew for the warning...such that it could be operated instinctively, inadvertently, or by habitual reflexive action. The system used to generate the aural warning must be designed to eliminate false or inappropriate alerts."

\section{Section 25.771 states:}

"(a) Each pilot compartment and its equipment must allow the minimum flight crew...to perform their duties without unreasonable concentration or fatigue...

(c) ...the airplane must be controllable with equal safety from either pilot seat.

(d) The pilot compartment must be constructed so that, when flying in rain or snow, it will not leak in a manner that will distract the crew...

(e) Vibration and noise characteristics of cockpit equipment may not interfere with safe operation of the airplane." 
Section 25.773 provides requirements for clear and undistorted view from the pilot compartment.

Section 25.777 requires:

“(a) Each cockpit control...[to] be located to provide convenient operation and to prevent confusion and inadvertent operation.

(b) The direction of movement of cockpit controls must... correspond to the sense of the operation upon the airplane or upon the part operated. Controls of a variable nature using a rotary motion must move clockwise from the off position , through an increasing range, to the full on position.

(c) The controls must be located and arranged, with respect to the pilots' seats, so that there is full and unrestricted movement of each control without interference for the cockpit structure or the clothing of the minimum flightcrew...

(d) Identical powerplant controls for each engine must be located to prevent confusion as to the engines they control.

(e) ...lift device controls must be located on top of the pedestal...

(f) The landing gear control must be located forward of the throttles and must be operable by each pilot when seated...

(g) Control knobs must be shaped in accordance with $\S 25.781$. In addition, the knobs must be of the same color, and this color must contrast with the color of control knobs for other purposes and the surrounding cockpit.

(h) If a flight engineer is required..., the airplane must have a flight engineer station located and arranged so that the flight crewmembers can perform their functions efficiently and without interfering with each other."

Section 25.779 prescribes requirements for the motion and effect of the cockpit controls.

Section 25.781 prescribes requirements for the shape of cockpit control knobs.

\section{Subpart E -- Powerplant}

Sections 25.1141, 25.1142, 25.1143, 25.1145, 25.1147, 25.1149, 25.1153, 25.1155, 25.1157, 25.1159 , and 25.1161 prescribe requirements for powerplant controls (general), auxiliary power unit controls, engine controls, ignition switches, mixture controls, propeller speed and pitch controls, propeller feathering controls, reverse thrust and propeller pitch settings below the flight regime, carburetor air temperature controls, supercharger controls, and fuel jettisoning system controls, respectively.

\section{Subpart F -- Equipment}

Section 25.1303 prescribes the flight and navigation instruments that are required.

Section 25.1305 prescribes the powerplant instruments that are required.

Section 25.1309 requires that 
"(b) The airplane systems and associated components... must be designed to that...the occurrence of...failure conditions which would reduce the...ability of the crew to cope with adverse operating conditions is improbable.

(c) Warning information must be provided to alert the crew to unsafe system operating conditions, and to enable them to take appropriate corrective action. Systems, controls, and associated monitoring and warning means must be designed to minimize crew errors which could create additional hazards.

(d) ...The [compliance] analysis must consider...the crew warning cues, corrective action required, and the capability of detecting faults."

Section 25.1321 prescribes arrangement and visibility requirements for flight, navigation, and powerplant instruments.

Section 25.1322 prescribes color requirements for warning, caution, and advisory lights.

Section 25.1329 states:

"(a) Each automatic pilot system must be approved and must be designed so that the automatic pilot can be quickly and positively disengaged by the pilots to prevent it from interfering with their control of the airplane.

(b) Unless there is an automatic synchronization, each system must have a means to readily indicate to the pilot the alignment of the actuating device in relation to the control system it operates.

(c) Each manually operated control for the system must be readily accessible to the pilots.

(d) Quick release (emergency) controls must be in both control wheels, on the side of each wheel opposite the throttles.

(e) Attitude controls must operate in the plane and sense of motion specified in $\S \S 25.777$ (b) and 25.779(a) for cockpit controls. The direction of motion must be plainly indicated on, or adjacent to, each control.

(f) The system must designed and adjusted so that, within the range of adjustment available to the human pilot, it cannot....create hazardous deviations in the flight path under any condition of flight appropriate to its use, either during a normal operation or in the event of a malfunction, assuming that corrective action begins within a reasonable period of time.

(g) If the pilot integrates signals from auxiliary controls or furnishes signals for operation of other equipment, there must be positive interlocks and sequencing of engagement to prevent improper operation...

(h) If the automatic pilot system can be coupled to airborne navigation equipment, means must be provided to indicate to the flight crew the current mode of operation. Selector switch position is not acceptable as a means of indication."

Section 25.1335 states that "If a flight director system is installed, means must be provided to indicate to the flight crew its current mode of operation. Selector switch position is not acceptable as a means of indication." 


\section{Subpart G -- Operating Limitations and Information}

Section 25.1523 states "The minimum flight crew must be established so that it is sufficient for safe operation, considering--

(a) The workload on individual crewmembers;

(b) The accessibility and ease of operation of necessary controls by the appropriate crewmember; and

(c) The kinds of operation authorized under $\S 25.1525$.

The criteria used in making the determinations required by this section are set forth in Appendix D

Section 25.1541 prescribes general requirements for markings and placards, while $\S 25.1543$ (b) states that "Each instrument marking must be clearly visible to the appropriate crewmember."

Section 25.1545 states that "The airspeed limitations required by $\S 25.1583$ (a) must be easily read and understood by the flight crew."

Appendix D to part 25 prescribes the criteria for determining the minimum flight crew, which consists of evaluations of crew workload factors.

\section{FAA Advisory Circulars (AC)}

AC 20-57A, "Automatic Landing Systems" sets forth an acceptable means of compliance for installation approval of systems for Category II operations. This AC states that malfunction of the automatic landing system should not:

(1) Cause significant displacement of the aircraft from its approach path, including altitude loss.

(2) Upon system disconnection, involve any out of trim condition not easily controlled by the pilot.

(3) Cause any action of the flight control system that is not readily apparent to the pilot, either by control movement or advisory display.

Also, a "means should be provided to inform the pilot continuously of the mode of operation of the automatic landing system. Indication of system malfunction should be conspicuous and unmistakable. Positive indication should be provided that the flare has (or alternatively has not) been initiated at the minimum normal flare engage heights."

AC 20-88A, "Guidelines on the Marking of Aircraft Powerplant Instruments (Displays)"

AC 25-11, "Transport Category Airplane Electronic Display Systems" covers a number of human factors issues. One item of interest is in paragraph $5 \mathrm{~g}$ on "Attention-Getting Requirements." It states, "For the displayed information to be effective as an attention-getter, some easily noticeable change must be evident. A legend change by itself is inadequate to annunciate automatic or uncommanded mode changes. Color changes may seem adequate in low light levels or during laboratory demonstrations but become much less effective at high 
ambient light levels. Motion is an excellent attention-getting device. Symbol shape changes are also effective, such as placing a box around freshly changed information. Short-term flashing symbols (approximately 10 seconds or flash until acknowledge) are effective attention-getters. A permanent or long-term flashing symbol that is noncancellable should not be used."

AC 25-15, “Approval of Flight Management Systems in Transport Category Airplanes." Some excerpts include: "Adequate annunciation of an impending automatic departure from a cruise altitude should be provided for all systems having vertical navigation modes. Systems that provide vertical navigation modes that automatically capture a preprogrammed vertical profile should require an overt flightcrew action within 5 minutes of the top of descent/bottom of climb point to activate the descent/climb unless the system is mechanized to the altitude selector in such a manner that a transition to descent or climb will not occur unless the altitude selector has been reset by the flightcrew to the new altitude. In addition, the system should contain design features that provide overspeed/underspeed protection (may be included in the autopilot system)."

"When the aircraft is being controlled in pitch, roll, thrust or airspeed by FMS functions, the annunciation of these modes or submodes of FMS operation shall be presented in a clear and unambiguous manner in the flightcrew's primary field of view."

"The airplane performance following addition of go-around thrust, during the landing approach go-around maneuver, with or without the simultaneous loss of an engine, should be such that the indicated airspeed is not reduced below that which existed upon initiation of the maneuver."

AC 25.1309-1A, "System Design and Analysis" includes guidance regarding the effect of system failures on flightcrew workload.

AC 25.1329-1A, "Automatic Pilot Systems Approval” provides an acceptable means of compliance with the requirements relating to malfunctions of the automatic pilot. 


\section{Appendix F Excerpts from the Aviation Safety Reporting System}

"The climb rate the autopilot had....nearly resulted in a stall as the aircraft bled its speed to maintain the climb [rate]. I relied too much on the autopilot and allowed myself to become distracted with my chart review. I'll never underestimate the potential for disaster that overreliance on an autopilot holds again."

"With autothrottles, flight level change feeds in power gradually. Using manual throttles, setting full climb power and hitting flight level change was too much [causing a speed excursion]. I have been on advance/automated aircraft for about 12 years and basic flying skills have deteriorated somewhat, [using] autothrottles causes you not to know basic power settings, etc."

"Captain was new to this 'high tech' 737-300 as well as newly upgraded. I had spent the past half hour showing him some ways of using the FMC navigation system, which he was not very familiar with...Finally he realized his mistake and had me ask for a visual [approach]. The airport... was not in our FMC database and I had to 'build it' for him (he didn't know how) -then got into a long in-flight discussion of how to build the OM using a radial and distance from ITU. This is info a check airman should have covered with him during IOE."

"Contributing factors in my opinion: ...The first officer is one of many dual-qualified pilots at our airline and I think it was a significant contributing factor...I can understand why his procedures and systems knowledge on my airplane are not the best. Finally, he and I have a company 'culture' difference. We come from two separate airlines that merged. The operating procedures that prevail now are predominantly from my 'culture.' I thought that he was somewhat resentful and less than enthusiastic about staying with those procedures, especially when I suggested that his procedures were not all standard or appropriate for this airplane."

"Why did the FMS drop the fix/restriction? I don't really know."

"The FMC is something that takes a lot of hands on experience before a pilot gets much proficiency and speed on it and 6 months practice over the last 2 years is not very much."

"Too much emphasis was placed on programming the FMC."

"FMC can give you a false sense of security because it's always accurate. This time it was off 3-5 miles..."

"The...FMC deletes crossing altitudes whenever a runway is changed or different approach is selected at the destination. This is exactly what it is programmed to do, and in my opinion it is 
a very dangerous program. I constantly warn new pilots about this trap in the...FMC. It had now caught me."

"Needless to say, confusion was in abundance. There are just too many different functions that can control airspeed and descent rates, all of which can control the altitude capture."

"My first priority was data entry rather than situational awareness."

"The first officer was too concerned about the FMC entry instead of starting the descent properly."

"I was lulled into complacency because I thought the FMC was properly programmed."

"This is a real trap...I was well aware of it and still got distracted."

"My inexperience led me to attempt to generate a computer solution for a simple manual VOR problem. Attempting to reduce the workload though automation created a more demanding situation, distracting us from the basics of flying."

"Also I have had no formal training on building restrictions in the computer from my company."

"Being new in an automated cockpit, I find that pilots are spending too much time playing with the computer in critical times rather than flying the aircraft."

"As routine as this flying becomes, it is easy to get in a trap of trusting the 'magic' of the glass cockpits instead of the old reliable raw data."

"I won't again attempt a CIVET profile using autopilot/FMC. The attention of both pilots is severely diluted by FMC operation and slow response."

"No amount of technology relieves the pilots of their duties of basic airmanship. One of the mistakes I made was assuming that after the aircraft captured VNAV PATH in the descent that it would make the crossing restriction and require no supervision. Technological advancements have in my opinion greatly enhanced and improved virtually all facets of aviation; however, errors will still be made by both the machinery and the pilots who control the machinery and in this particular incident, complacency was certainly a factor."

"This is not an isolated case. I have experienced similar scenarios before. We spend hours doing nothing at cruise while the electronic wonderware does all. Near the airport, the wonderware fails, the airport equipment and personnel put unnecessary burdens on us...which can lead to very serious consequences."

"I reselected VNAV and the aircraft responded with a very dramatic nose down pitch. You can get busy real fast when all the magic fails that close to landing. I hope new low-time 
people moving almost directly into glass cockpits don't rely too much on automation and forget how to fly. My opinion -- it's great, but don't ever trust it."

"This event involves a feeling of complacency brought on by the latest generation of highly automated, glass-cockpit airplanes. The capability to fully program complex procedures can lead to a perception on the part of the flightcrew that the flight management system, once programmed, will follow a particular procedure fully and completely."

"Often when making descents with rapidly changing parameters, the automated cockpit becomes unwieldy -- and becomes a distraction to flying the aircraft."

"The automation is great under normal conditions and works well when you have the time to monitor. When there isn't time to monitor, you need to fly the airplane without deliberately trying to override systems that were never designed to perform these nonstandard takeoff profiles."

"Had he continued to follow the flight director, we would have had a full power stall in IFR conditions...I believe we are slowly working ourselves into detrimental reliance on FMS/glass cockpits/autoflight systems."

"The cause of this uncommanded climb was never determined by the crew and did not result in any traffic conflict our knowledge. Taking into account the complexity of the FMC and its ability to revert automatically from one mode to another as well as the high cockpit workload at this point, one has no time to try and diagnose the reason behind an unwanted autopilot action and disconnection is the only prudent action."

"I've learned that the more gizmos installed (FMC, TCAS, ACARS, etc.), the less time you have to devote to the primary job of flying the aircraft."

"The first officer seemed at first confused about how to manage the MCP best to comply with the speed and altitude requirement. He was using the vertical speed mode to slow his descent to reach the requested speed and when he heard the call to expedite descent, he deployed speedbrakes, called for flaps to $15 \mathrm{deg}$ and forgot the MCP was in vertical speed; therefore, got no increase in descent rate. Actually, the autothrottle added power to maintain selected speed at his selected vertical speed."

"Concentration on automation rather than just flying the aircraft was enough distraction to fly through the altitude."

"There are problems with training devices that have programming and logic discrepancies. Many training programs do not adequately address the real time environment."

"I was led down the primrose path relying too heavily on the normally reliable programmed FMC computer, great as long as correct." 
"While we were in steady state level flight, the go-around button was hit by accident by a clip board; this caused the autopilot to disengage and the throttles to advance to takeoff thrust. Following the confusion of the moment, the aircraft started to climb...By the time we reengaged the autopilot and stopped the climb, we had gained about 400 feet."

"Note that on the advanced cockpit airplane with the side stick controller, the PNF has no idea of the other pilot's control movements, but can only rely on the aircraft pitch and roll angle changes. This makes it very difficult to know what to interject during a potential bad landing."

"Rather than ignore the map and concentrate on raw data, the captain was playing catch-up with the computer."

"I failed to realize that the altitude restrictions are not in effect during a speed mode descent."

"We descended 400 feet below the 10000 foot crossing altitude...How did this happen? I suppose the wizardry of the 'glass cockpit' and two newcomers who were not aggressive enough intervening when the computer did other than what they expected."

"The nature of these machines, unlike a pilot, is to maintain a high rate of descent until the capture zone, then make a rather abrupt level off."

"With over 4000 hours in advanced cockpits, I have found that these supposedly 'fail safe' systems can occasionally set us up and then let us down in a big way."

"Automated flight holds many traps for us, most of which are altitude related. We must be even more vigilant than we were before."

“The captain then said, 'What's going on?' at which point the aircraft was observed 300 feet high; it had entered a subtle climb seemingly on its own accord...This is another case of learning to type 80 words a minute instead of flying the aircraft. The more automation there is in the aircraft, it just means the flightcrew should work that much harder to remain an active and integral part of the loop."

"Heading select knob doubles as heading hold button and an imperceptible extra push in on it activates heading hold. Multifunction knobs should not be accepted on aircraft. It is simply too easy at night when you are tired or distracted to activate the wrong function."

"Both of us were engrossed in trying to figure out why this computerized marvel was doing what it was, rather than turning everything off and manually flying (which we finally did) until we could sort things out."

"We entered the altitude change, began descent, and were playing the 'what's it doing now' game to determine why it hadn't slowed down as commanded. Time lost trying to decide what it was up to put us behind the aircraft." 
"The captain was confused at this point...and I could not get him to abandon the computer."

"Controllers need to understand the increase in workload that is placed on a 2-man crew using an FMC when giving restrictions and holding instructions off of a fix not on their route."

"I don't know why, with everything apparently set in properly, the aircraft did not descend at the proper time. I feel the cause of this mistake is too much reliance on automated systems and a lack of vigilance on my part as to the altitude and position of my aircraft."

"I do not believe that ATC controllers understand the operation of computer driven aircraft. We are plagued with late clearances, frequent changes...Also, it would be nice if the center used the enroute waypoints instead of giving us mileage points...These simple changes to procedures would help cut out workload so we could keep our heads out of the cockpit and still use the computer."

"The flight management computer can be mis-programmed and look exactly right, unless one is trained to be alert for potential programming pitfalls."

"The captain began programming the FMC when we should have started right down to FL190...We relied too much on the FMC's in a situation where they require too much input and monitoring and increase the workload."

"The co-pilot was relatively new on the aircraft and relied too much on the VNAV capabilities of the autopilot, and this resulted in being 300-500 feet above the...altitude restriction."

"I diverted my attention from the basic responsibility of flying the aircraft to attend to the intricacies of reprogramming the computer."

"We've become so used to using the EFIS map mode and autoflight that we've gotten away from VOR navigation. Partly complacency, partly confusion."

"Also, both of these situations could have been prevented if we had not depended so much on the automation and gone back to basic flying."

"But I was so preoccupied with reprogramming the FMC that his warnings didn't register with me."

"A lack of practical experience utilizing this equipment causes these deviations, and the time delay in computer spool up to divulge the info is also critical."

"We missed the crossing altitude by 1000 feet. The captain was...busy trying to program the FMC. Being new in an automated cockpit, I find that pilots are spending too much time playing with the computer at critical times rather than flying the aircraft. No one looks outside for traffic." 
"The autothrottle was adding power, and I was fighting it and pulling the throttle back. Finally, I shut off the autothrottle...It took me a moment to realize the autothrottle was not engaged and the aircraft slowed to the stickshaker as we leveled."

"A 3000 foot error. Captain did tell me that he does not like this automatic cockpit stuff. Perhaps pilots who are that uncomfortable with new generation aircraft should stay with steam gauges, or train more..." 


\section{Appendix G \\ Questions Used to Guide Discussions with Manufacturers and Operators}

\section{FAA Human Factors Study Team Outline of Areas of Interest}

1) Design issues about crew-system interfaces that potentially affect safe flight path management (generically or by specific, airplane type).

2) Implications of glass cockpit airplanes for training and crew qualifications.

3) Implications of glass cockpit airplanes for line operations (practices, procedures, checklists, company policies)

4) Role and suitability of FAA, JAA, and industry safety standards and policies.

5) Role and suitability of industry-wide processes to identify and resolve issues related to safe flight path management of glass cockpit airplanes 


\section{Questions To Guide The Discussion With Operators}

\section{$\underline{\text { Operations }}$}

1. Do you have an overall philosophy for the use of flight deck automation? If so, what is it?

2. Did you develop this philosophy from the equipment that you had, or did you acquire equipment that fit this philosophy?

3. What issues do you see, if any, in the need to create operating policies, procedures, or checklists to supplement or compensate for design characteristics of your glass cockpit airplanes?

\section{Training}

1. What training philosophy do you adhere to, with respect to the use of automation and flight path management in glass cockpits?

2. Have there been modifications to training to accommodate different automation designs?

3. Can you identify any generic issues that affect crew qualification (e.g. training, checking, or recency of experience) that may need to be addressed industry-wide for all glass cockpit aircraft, or industry-wide for a particular type of glass cockpit airplanes?

\section{$\underline{\text { Design }}$}

1. Have your crews experienced automation or flight path surprises, or mode confusion? What design characteristics, if any, have contributed to these?

2. In terms of flight deck design, do you have concerns about:

2.1. Any specific aircraft type?

2.2. Transfer of pilots between particular types?

2.3. Particular types in certain settings, (e.g., at certain airports, or certain ATC systems, or certain weather conditions)? 
3. What design issues or characteristics are you aware of, generically or by specific airplane type, that may be unduly contributing to difficulties regarding safe flight path management?

\section{Standards, Policies, Processes And Research}

1. What areas do you believe the Authorities should change in the standards, policies or processes to assure safer operation of present and future glass cockpit airplanes?

2. What areas do you believe the industry (manufacturing, operating, etc.) should change in the standards, policies or processes to assure safer operation of present and future glasscockpit airplanes?

3. What areas do you think should be addressed in research to improve design and operation of present and future glass cockpit airplanes?

4. What else do you think this Team should consider about design, training and operation of glass cockpit airplanes?

5. Do you have any additional suggestions for the Team in this or other areas?

\section{Questions To Guide The Discussions With The Manufacturers}

Please be prepared to discuss the following questions. Include, as applicable, the specific supporting studies, data, etc.

\section{Design Philosophy}

1. Please describe your overall automation philosophy and how it has evolved.

- How do you decide which tasks get automated?

- How are different operating environments (e.g., air traffic systems, operator practices, cultures) taken into account?

- What specific studies, data sources, etc. have affected your automation philosophy and in what way?

2. When you learn of difficulties in service, how do you decide whether to introduce a design change, an operators bulletin, or a product improvement?

3. What changes in flight deck interface design do you see occurring in the future? 


\section{$\underline{\text { Design Process }}$}

1. Please describe your flight deck design process, particularly in terms of the automated flight path management functions and the interfaces between the flightcrew and the automation.

2. In what ways do your customers influence the design, both in terms of the functions provided and how they are implemented?

3. How are human factors issues identified and resolved?

- At what point in the design process does this occur?

- What standards and methods do you use to test and evaluate human performance?

4. How are training considerations taken into account or anticipated during design?

\section{$\underline{\text { Design Features }}$}

1. How do you decide what autoflight modes to include?

2. How do you allocate tasks between crew members, and how do you incorporate that into the design?

\section{Service Implementation}

1. What training philosophy (or philosophies) do you recommend in regards to using the automation to manage the flight path?

2. Are there any generic issues involving crew qualification (e.g., training, checking, recency of experience) that should be addressed for either for all types or for a particular type of glass cockpit airplane?

3. What processes do you use to ensure the adequacy and accuracy of training tools and manuals?

4. How do you obtain and incorporate feedback from your customers?

\section{$\underline{\text { In-Service Issues }}$}

Please address the following areas of interest:

1. Crew awareness/feedback

- Mode awareness

- Mode changes

- Flight control positions

- Failures of the automatic systems 
- Behavior of the automatic systems

- Trim setting and trim changes

- Thrust setting and thrust changes

2. Standardization of the automation interfaces

3. Envelope protection

4. Autopilot/Autothrottle

- Methods of engaging and disconnecting

- Mode selection (direct and indirect)

- Autopilot trim authority

- Force disconnects

5. Vertical Navigation

6. Crew Workload/Boredom/Complacency/Fatigue

7. Display clutter

\section{$\underline{\text { Standards, Policies, Processes, and Research }}$}

1. In what areas, if any, should the regulatory authorities change the current standards, policies, or processes in order to assure safe operation of present and future glass cockpit airplanes?

2. In what areas, if any, should the industry (including manufacturers and operators) change the current standards, policies, or processes in order to assure safe operation of present and future glass cockpit airplanes?

3. What areas, if any, should be addressed by research in order to assure safe operation of present and future glass cockpit airplanes?

4. What else do you think this Team should consider about the design, training, and operation of glass cockpit airplanes?

5. Do you have any additional suggestions for the Team in this or other areas? 